M.-A. Inaba

Nagoya Math. J.

Vol. 166 (2002), 135-181

\title{
ON THE MODULI OF STABLE SHEAVES ON A REDUCIBLE PROJECTIVE SCHEME AND EXAMPLES ON A REDUCIBLE QUADRIC SURFACE
}

\author{
MICHI-AKI INABA
}

\begin{abstract}
We study the moduli space of stable sheaves on a reducible projective scheme by use of a suitable stratification of the moduli space. Each stratum is the moduli space of "triples", which is the main object investigated in this paper. As an application, we can see that the relative moduli space of rank two stable sheaves on quadric surfaces gives a nontrivial example of the relative moduli space which is not flat over the base space.
\end{abstract}

\section{Introduction}

Simpson has constructed the moduli scheme of stable sheaves on an arbitrary projective scheme ([17]). This result causes us to study many examples of moduli spaces of stable sheaves on degenerate varieties. A typical example is the moduli space of sheaves on a nodal curve studied by Seshadri ([16]). He showed that the moduli space has the singularity similar to that of base curve. On the other hand, Gieseker and Li used the moduli space of stable sheaves on a reducible surface in order to prove the irreducibility of the moduli space of rank 2 stable bundles on a smooth surface $([3])$. This result tells us an importance of the study of the moduli spaces of stable sheaves on reducible schemes.

In this paper we shall study the moduli space of stable sheaves on a reducible projective scheme $X=X_{1} \cup X_{2}$ such that $X_{1}$ and $X_{2}$ are purely $d$-dimensional and $Y:=X_{1} \cap X_{2}$ is a Cartier divisor of $X_{1}$ and $X_{2}$. In order to study the moduli space, we shall use a generalization of the method of Nagaraj and Seshadri ([11]), which was used on reducible curves. We shall show that there is a bijective correspondence between the purely $d$ dimensional coherent sheaves on $X$ and the triples $\left(E_{1}, \tilde{E}_{2}, f\right)$ on $X$, where $E_{1}$ is a purely $d$-dimensional sheaf on $X_{1}, \tilde{E}_{2}$ a purely $d$-dimensional sheaf on $X_{2}$ and $f:\left.\left.E_{1}\right|_{Y} \rightarrow \tilde{E}_{2}\right|_{Y}$ a homomorphism. In Theorem 1.10, we will

Received June 28, 2000.

2000 Mathematics Subject Classification: 14D20, 14D06, 14J60. 
show that the moduli space of triples exists and that the moduli spaces of triples give a stratification of the moduli space of stable sheaves on $X$. This stratification is a valuable tool for the study of the moduli space of stable sheaves on a reducible scheme. In fact we can investigate a more precise structure of the moduli space of triples from the construction given in Theorem 2.1. From this construction, one sees that the moduli space of triples has a fibration in étale topology whose fibers are open subschemes of projective spaces. Although the dimension of the fibers of this fibration is constant in the case of reducible curves, the dimension of fibers may jump in higher dimensional case (Remark 2.2). On the other hand, there is a bijective correspondence between the purely $d$-dimensional coherent sheaves on $X$ and the "parabolic triples" defined in Definition 3.1. The moduli space of parabolic triples is an intersection of two moduli spaces of triples.

As an application of the study of the moduli space of triples, we can construct a non-trivial example of the relative moduli space of stable sheaves which is not flat over the base scheme. We shall see an example of the decomposition of the moduli space of rank 2 stable sheaves on a reducible quadric surface by the moduli spaces of triples (Theorem 5.1). From this, one sees that there are components of the moduli space whose dimension is jumping. Moreover we shall study the deformations of sheaves on reducible surfaces and apply it to degenerations of quadric surfaces. Then we can see that "general" points of the moduli space on a reducible quadric surface are contained in the limits of stable sheaves on smooth quadric surfaces (Theorem 5.4 and Conclusion 5.5). From this point of view, one recognizes that the concept of stable sheaf introduced by [17] makes a good sense in this case.

The author would like to thank Professors Masaki Maruyama and Akira Ishii for valuable discussions and encouragement.

\section{Notation and convention}

Let $X$ be a projective scheme over a noetherian scheme $S, \mathcal{O}_{X}(1)$ an $S$ very ample invertible sheaf and $E$ a coherent sheaf on $X$. (Sch/S) denotes the category of locally noetherian schemes over $S$ and (Sets) the category of sets. For an integer $m, E(m)$ denotes $E \otimes \mathcal{O}_{X}(m)$. If $s$ is a point of $S$, then we denote the fiber of $X$ over $s$ by $X_{s}, E \otimes k(s)$ by $E(s), \operatorname{dim} H^{i}\left(X_{s}, E(s)\right)$ by $h^{i}(E(s))$ and $\sum_{i \geq 0}(-1)^{i} h^{i}(E(s))$ by $\chi(E(s))$. For a morphism $g: T \rightarrow S$ of schemes, $E_{T}$ denotes the sheaf $\left(1_{X} \times g\right)^{*}(E)$ on $X \times_{S} T$. If $F$ is a 
coherent sheaf on $S, \mathbf{P}(F)$ means Proj $S(F)$ and $\mathbf{V}(F)$ means $\operatorname{Spec} S(F)$, where $S(F)$ is the symmetric algebra of $F$ over $\mathcal{O}_{S}$. For a polynomial $H(x)$ and an integer $m, H[m](x)$ is the shift of $H(x)$ by $m$.

\section{\$1. Fundamental properties of the moduli spaces of stable sheaves on reducible projective schemes}

Throughout this paper, we fix an algebraically closed field $k$.

Definition 1.1. Let $E$ be a non-zero coherent sheaf on an algebraic scheme $S$ over $k$. Then $E$ is said to be of pure dimension $d$ if $\operatorname{dim}(\operatorname{Supp} F)=$ $d$ for any non-zero coherent subsheaf $F$ of $E$.

In this section we will consider a projective scheme $X$ over $k$ with the following properties:

$X=X_{1} \cup X_{2}$ where $X_{i}(i=1,2)$ are closed subschemes of $X$ such that $\mathcal{O}_{X_{i}}$ are of pure dimension $d$ for $i=1,2$ and $I_{X_{1}} \cap I_{X_{2}}=0$,

$(\dagger) \quad$ where $I_{X_{i}}$ is the ideal sheaf of $\mathcal{O}_{X}$ corresponding to the closed subscheme $X_{i}$, and $Y:=X_{1} \cap X_{2}$ is a Cartier divisor of $X_{1}$ and $X_{2}$ at the same time.

Note that there is a canonical exact sequence

$$
0 \longrightarrow \mathcal{O}_{X} \longrightarrow \mathcal{O}_{X_{1}} \oplus \mathcal{O}_{X_{2}} \longrightarrow \mathcal{O}_{Y} \longrightarrow 0
$$

Since $\mathcal{O}_{X_{1}}$ and $\mathcal{O}_{X_{2}}$ are of pure dimension $d, \mathcal{O}_{X}$ is also of pure dimension $d$.

We will give a description of purely $d$-dimensional sheaves on $X$ by data on $X_{1}, X_{2}$. Let $E$ be a coherent sheaf of pure dimension $d$ on $X$. Put

$$
E^{(1)}:=\left(\left.E\right|_{X_{1}}\right) /\left(\left.E\right|_{X_{1}}\right)_{\text {tor }}, \quad E^{(2)}:=\left(\left.E\right|_{X_{2}}\right) /\left(\left.E\right|_{X_{2}}\right)_{\text {tor }},
$$

where $\left(\left.E\right|_{X_{i}}\right)_{\text {tor }}$ is the coherent subsheaf of $\left.E\right|_{X_{i}}$ such that $\operatorname{dim} \operatorname{Supp}\left(\left.E\right|_{X_{i}}\right)_{\text {tor }}$ $<d$ and $\left(\left.E\right|_{X_{i}}\right) /\left(\left.E\right|_{X_{i}}\right)_{\text {tor }}$ is of pure dimension $d$. There is a canonical injection $i: E \rightarrow E^{(1)} \oplus E^{(2)}$. If we put $E^{(0)}:=$ coker $i$, then we have the following exact sequence;

$$
0 \longrightarrow E \stackrel{i}{\longrightarrow} E^{(1)} \oplus E^{(2)} \longrightarrow E^{(0)} \longrightarrow 0 .
$$

LEMMA 1.2. $E^{(0)}$ is an $\mathcal{O}_{Y}$-module. 
Proof. The composite $I_{X_{2}} \hookrightarrow \mathcal{O}_{X} \rightarrow \mathcal{O}_{X_{1}}$ is injective and the image is just the ideal sheaf $\mathcal{O}_{X_{1}}(-Y)$ corresponding to the Cartier divisor $Y$ of $X_{1}$. Tensoring $E$ to the injection $I_{X_{2}} \hookrightarrow \mathcal{O}_{X}$, we have a homomorphism

$$
\left.E\right|_{X_{1}} \otimes \mathcal{O}_{X_{1}}(-Y) \cong E \otimes I_{X_{2}} \longrightarrow E
$$

Since $E$ is of pure dimension $d$, the above homomorphism induces the homomorphism $E^{(1)} \otimes \mathcal{O}_{X_{1}}(-Y) \rightarrow E$. The composition $E^{(1)} \otimes \mathcal{O}_{X_{1}}(-Y) \rightarrow E \rightarrow$ $E^{(1)}$ is just the canonical homomorphism. Note that $E^{(1)} \otimes \mathcal{O}_{X_{1}}(-Y) \rightarrow$ $E^{(1)}$ is injective since $E^{(1)}$ is of pure dimension $d$. We denote $E^{(1)} \otimes$ $\mathcal{O}_{X_{1}}(-Y)$ by $E^{(1)}(-Y)$. Similarly we have a canonical injection $E^{(2)}(-Y)$ $\hookrightarrow E$. Let $(a, b)$ be a local section of $E^{(1)} \oplus E^{(2)}$ and $c$ a local section of $I_{Y}$, where $I_{Y}$ is the ideal sheaf of $\mathcal{O}_{X}$ corresponding to the closed subscheme $Y$ of $X$. Then $c$ can be written as $c=c_{1}+c_{2}$, where $c_{1} \in I_{X_{1}}$ and $c_{2} \in I_{X_{2}}$. So $c \cdot(a, b)=\left(c_{2} a, c_{1} b\right)$. Since $c_{2} a \in E^{(1)}(-Y) \subset E$ and $c_{1} b \in E^{(2)}(-Y) \subset E$, we have $c \cdot(a, b) \in E$. Hence $I_{Y}\left(E^{(1)} \oplus E^{(2)}\right) \subset E$ and so $I_{Y} E^{(0)}=0$.

Let $p$ be the composition:

$$
\begin{aligned}
& p: E^{(2)} \hookrightarrow E^{(1)} \oplus E^{(2)} \longrightarrow E^{(0)} . \\
& a \quad \mapsto \quad(0, a)
\end{aligned}
$$

Then we can easily see that $p$ is surjective. Now we put $\tilde{E}^{(2)}:=\operatorname{ker} p \otimes$ $\mathcal{O}_{X_{2}}(Y)$. Since $E^{(0)}$ is an $\mathcal{O}_{Y}$-module, the composite

$$
E^{(2)}(-Y) \hookrightarrow E^{(2)} \stackrel{p}{\longrightarrow} E^{(0)}
$$

is zero. So we have a factorization

$$
E^{(2)}(-Y) \hookrightarrow \tilde{E}^{(2)}(-Y)=\operatorname{ker} p \hookrightarrow E^{(2)} .
$$

Tensoring $\mathcal{O}_{X_{2}}(Y)$ to the injection $E^{(2)}(-Y) \hookrightarrow \tilde{E}^{(2)}(-Y)$, we have an injection $E^{(2)} \hookrightarrow \tilde{E}^{(2)}$ and the composition $\tilde{E}^{(2)}(-Y) \hookrightarrow E^{(2)} \hookrightarrow \tilde{E}^{(2)}$ is just the canonical injection obtained from the injection $\mathcal{O}_{X_{2}}(-Y) \hookrightarrow \mathcal{O}_{X_{2}}$. Since the composite $\operatorname{ker} p=\left.\tilde{E}^{(2)}(-Y) \rightarrow E^{(2)} \rightarrow \tilde{E}^{(2)} \rightarrow \tilde{E}^{(2)}\right|_{Y}$ is zero, there exists a homomorphism $j:\left.E^{(0)} \rightarrow \tilde{E}^{(2)}\right|_{Y}$ such that the following diagram commutes:

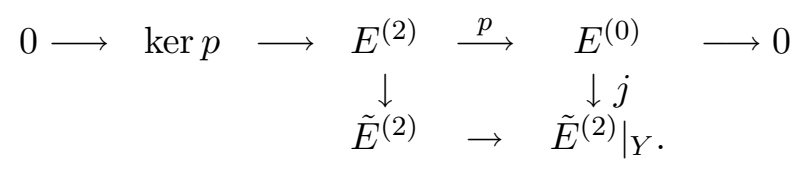


Let us consider the composition $f_{E}:\left.\left.E^{(1)}\right|_{Y} \rightarrow E^{(0)} \stackrel{j}{\rightarrow} \tilde{E}^{(2)}\right|_{Y}$ and put

$$
\varphi_{f_{E}}:\left.E^{(1)} \oplus \tilde{E}^{(2)} \rightarrow \tilde{E}^{(2)}\right|_{Y} ; \quad \varphi_{f_{E}}(a, b):=f_{E}\left(\left.a\right|_{Y}\right)-\left.b\right|_{Y}
$$

We have a canonical injection $E \hookrightarrow E^{(1)} \oplus E^{(2)} \hookrightarrow E^{(1)} \oplus \tilde{E}^{(2)}$.

LEMMA 1.3. With respect to the above injection $E \hookrightarrow E^{(1)} \oplus \tilde{E}^{(2)}$, we have $E=\operatorname{ker} \varphi_{f_{E}}$.

Proof. Since the diagram

$$
\begin{array}{rlll}
0 \longrightarrow E \longrightarrow E^{(1)} \oplus E^{(2)} & \longrightarrow & E^{(0)} \\
& \downarrow & & \downarrow j \\
& E^{(1)} \oplus \tilde{E}^{(2)} & \stackrel{\varphi_{f_{E}}}{\longrightarrow} & \left.\tilde{E}^{(2)}\right|_{Y}
\end{array}
$$

commutes, the composite $\left.E \hookrightarrow E^{(1)} \oplus \tilde{E}^{(2)} \stackrel{\varphi_{f_{E}}}{\longrightarrow} \tilde{E}^{(2)}\right|_{Y}$ is zero. So we have the inclusion $E \subset \operatorname{ker} \varphi_{f_{E}}$. Conversely let $(a, b) \in \operatorname{ker} \varphi_{f_{E}} \subset E^{(1)} \oplus \tilde{E}^{(2)}$ be a local section. There exists a local section $\alpha \in E$ such that $\left.\alpha\right|_{X_{1}}=a$ in $E^{(1)}$. If we put $b^{\prime}:=b-\left.\alpha\right|_{X_{2}}\left(\in \tilde{E}^{(2)}\right)$, then $\left(0, b^{\prime}\right)=(a, b)-i(\alpha) \in \operatorname{ker} \varphi_{f_{E}}$ and so we have only to prove that $\left(0, b^{\prime}\right) \in E$. Since $0=\varphi_{E}\left(0, b^{\prime}\right)=\left.b^{\prime}\right|_{Y}$ in $\left.\tilde{E}^{(2)}\right|_{Y}, b^{\prime} \in \tilde{E}^{(2)}(-Y)=\operatorname{ker} p \subset E^{(2)}$. Hence $\left(0, b^{\prime}\right)$ is in the kernel of the homomorphism $E^{(1)} \oplus E^{(2)} \rightarrow E^{(0)}$ and so $\left(0, b^{\prime}\right) \in E$.

Definition 1.4. Let $E_{1}$ (resp. $\tilde{E}_{2}$ ) be a coherent sheaf of pure dimension $d$ on $X_{1}$ (resp. $X_{2}$ ). Let $f:\left.\left.E_{1}\right|_{Y} \rightarrow \tilde{E}_{2}\right|_{Y}$ be a homomorphism. Then we call $\left(E_{1}, \tilde{E}_{2}, f\right)$ a triple. Two triples $\left(E_{1}, \tilde{E}_{2}, f\right),\left(E_{1}^{\prime}, \tilde{E}_{2}^{\prime}, f^{\prime}\right)$ are said to be isomorphic if there exist isomorphisms $g_{1}: E_{1} \stackrel{\sim}{\rightarrow} E_{1}^{\prime}, g_{2}: \tilde{E}_{2} \stackrel{\sim}{\rightarrow} \tilde{E}_{2}^{\prime}$ such that the diagram

$$
\begin{array}{rrr}
\left.E_{1}\right|_{Y} & \stackrel{f}{\longrightarrow} & \left.\tilde{E}_{2}\right|_{Y} \\
\left.g_{1}\right|_{Y} \downarrow l & & \left.g_{2}\right|_{Y} \downarrow \text { ᄂ } \\
\left.E_{1}^{\prime}\right|_{Y} & \stackrel{f^{\prime}}{\longrightarrow} & \left.\tilde{E}_{2}^{\prime}\right|_{Y}
\end{array}
$$

commutes.

The following proposition is a generalization of [[11], Lemma 2.3].

Proposition 1.5. $E \mapsto\left(E^{(1)}, \tilde{E}^{(2)}, f_{E}\right)$ is a bijective correspondence between the isomorphism classes of coherent sheaves of pure dimension $d$ on $X$ and the isomorphism classes of triples. 
Proof. Take a triple $\left(E_{1}, \tilde{E}_{2}, f\right)$. Put

$$
\varphi_{f}:\left.E_{1} \oplus \tilde{E}_{2} \rightarrow \tilde{E}_{2}\right|_{Y} ; \quad \varphi_{f}(a, b):=f\left(\left.a\right|_{Y}\right)-\left.b\right|_{Y}
$$

We will show that $\left(E_{1}, \tilde{E}_{2}, f\right) \mapsto \operatorname{ker} \varphi_{f}$ gives the inverse map. If we put $E:=\operatorname{ker} \varphi_{f}$, then we can construct the triple $\left(E^{(1)}, \tilde{E}^{(2)}, f_{E}\right)$. Since we know Lemma 1.3, we have only to prove that $\left(E^{(1)}, \tilde{E}^{(2)}, f_{E}\right) \cong\left(E_{1}, \tilde{E}_{2}, f\right)$. From the definition of $E^{(1)}$, the homomorphism $E \rightarrow E_{1}$ factors through $E^{(1)}$ :

$$
E \longrightarrow E^{(1)} \stackrel{\sigma_{1}}{\rightarrow} E_{1}
$$

By construction $E \rightarrow E_{1}$ is surjective. On the other hand, $\sigma_{1}$ is injective since it is injective on $X_{1} \backslash Y$ and $E^{(1)}$ is of pure dimension $d$. Thus $\sigma_{1}$ : $E^{(1)} \rightarrow E_{1}$ is an isomorphism. Put $\psi: \tilde{E}_{2}(-Y) \hookrightarrow \tilde{E}_{2} \hookrightarrow E_{1} \oplus \tilde{E}_{2}$. Then $\psi$ factors through $E$. Let $\psi^{\prime}: \tilde{E}_{2}(-Y) \rightarrow E$ be the induced homomorphism. Then

$$
0 \longrightarrow \tilde{E}_{2}(-Y) \stackrel{\psi^{\prime}}{\longrightarrow} E \longrightarrow E_{1} \longrightarrow 0
$$

becomes an exact sequence. On the other hand

$$
0 \longrightarrow \tilde{E}^{(2)}(-Y) \longrightarrow E \longrightarrow E^{(1)} \longrightarrow 0
$$

is also exact. So we have an isomorphism $\tilde{E}^{(2)}(-Y) \stackrel{\sim}{\rightarrow} \tilde{E}_{2}(-Y)$ with the following commutative diagram:

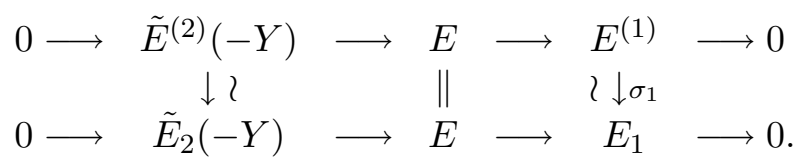

This isomorphism induces the isomorphism $\tilde{E}^{(2)} \stackrel{\sigma_{2}}{\rightarrow} \tilde{E}_{2}$ and the diagram

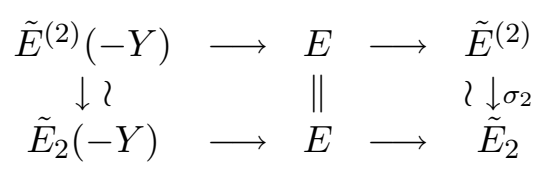

commutes. Since the diagrams

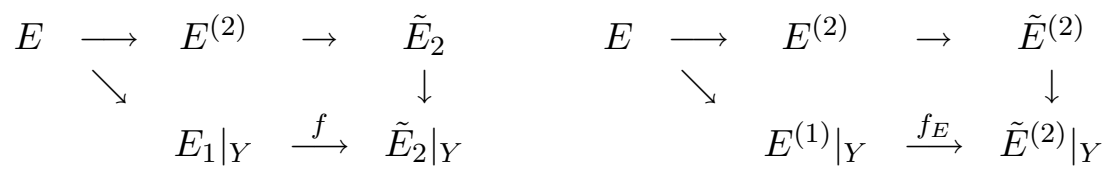


both commute, we can see that the diagram

$$
\begin{array}{ccc}
\left.E^{(1)}\right|_{Y} & \stackrel{f_{E}}{\longrightarrow} & \left.\tilde{E}^{(2)}\right|_{Y} \\
\text { ᄂ } \sigma_{1} & & \text { ? } \sigma_{2} \\
\left.E_{1}\right|_{Y} & \stackrel{f}{\longrightarrow} & \left.\tilde{E}_{2}\right|_{Y} .
\end{array}
$$

commutes. Thus we have an isomorphism $\left(E^{(1)}, \tilde{E}^{(2)}, f_{E}\right) \cong\left(E_{1}, \tilde{E}_{2}, f\right)$.

Now let us recall the definition of stable sheaf on $X$. We fix a very ample line bundle $\mathcal{O}_{X}(1)$ on $X$. Then the Hilbert polynomial of a coherent sheaf $E$ on $X$ with respect to $\mathcal{O}_{X}(1)$ can be written as

$$
\chi(E(m))=\sum_{i=0}^{d} a_{i}(E)\left(\begin{array}{c}
m+d-i \\
d-i
\end{array}\right),
$$

with $a_{i}(E)$ integers. We put $\mu^{S}(E):=a_{1}(E) / a_{0}(E)$.

Definition 1.6. Let $E$ be a coherent sheaf of pure dimension $d$ on $X$. $E$ is said to be stable (resp. semi-stable) if for any coherent subsheaf $F$ of $E$ with $0<a_{0}(F)<a_{0}(E)$,

$$
\begin{gathered}
\chi(F(m)) / a_{0}(F)<\chi(E(m)) / a_{0}(E) \\
(\text { resp. } \leq)
\end{gathered}
$$

for all sufficiently large integers $m$.

Let $(\mathrm{Sch} / k)$ be the category of locally noetherian schemes over $k$. Let $H, H_{1}, H_{2}, H_{1}^{\prime}, H_{2}^{\prime}$ be numerical polynomials of degree $d$ such that $H=$ $H_{1}+H_{2}^{\prime}$.

Definition 1.7. We define a functor $\mathcal{M}_{X}^{H}:(\mathrm{Sch} / k) \rightarrow($ Sets $)$ by

$$
\left.\begin{array}{rl}
\mathcal{M}_{X}^{H}(T):= & \left\{\begin{array}{l}
E \text { is a } T \text {-flat coherent sheaf on } X \times T \\
\text { such that } \chi(E(t)(m))=H(m) \text { and } \\
E(t) \text { is a stable sheaf for all } t \in T
\end{array}\right.
\end{array}\right\} / \sim
$$

where $E \sim E^{\prime}$ if and only if $E \cong E^{\prime} \otimes L$ for some line bundle $L$ on $T$. 
We define a functor $\mathcal{M}_{X, H_{1}^{\prime}, H_{2}^{\prime}}^{(1), H_{1}, H_{2}}:(\mathrm{Sch} / k) \rightarrow($ Sets $)$ by

$$
\begin{aligned}
& \mathcal{M}_{X, H_{1}^{\prime}, H_{2}^{\prime}}^{(1), H_{1}, H_{2}}(T):= \\
& \left\{\begin{array}{l|l}
\left(E_{1}, \tilde{E}_{2}, f\right) & \begin{array}{l}
E_{1} \text { is a } T \text {-flat coherent } \mathcal{O}_{X_{1} \times T} \text {-module, } \\
\tilde{E}_{2} \text { is a } T \text {-flat coherent } \mathcal{O}_{X_{2} \times T} \text {-module } \\
\text { and } f:\left.\left.E_{1}\right|_{Y \times T} \rightarrow \tilde{E}_{2}\right|_{Y \times T} \text { is a homo- } \\
\text { morphism with the property }(*)
\end{array}
\end{array}\right\} / \sim
\end{aligned}
$$

where $\left(E_{1}, \tilde{E}_{2}, f\right) \sim\left(E_{1}^{\prime}, \tilde{E}_{2}^{\prime}, f^{\prime}\right)$ if and only if there exist a line bundle $L$ on $T$ and isomorphisms $g_{1}: E_{1} \stackrel{\sim}{\rightarrow} E_{1}^{\prime} \otimes L, g_{2}: \tilde{E}_{2} \stackrel{\sim}{\rightarrow} \tilde{E}_{2}^{\prime} \otimes L$ with the following commutative diagram:

$$
\begin{array}{ccc}
\left.E_{1}\right|_{Y \times T} & \stackrel{f}{\longrightarrow} & \left.\tilde{E}_{2}\right|_{Y \times T} \\
\left.g_{1}\right|_{Y \times T} \downarrow \text { ᄂ } & & \left.g_{2}\right|_{Y \times T} \downarrow \text { ? } \\
\left.E_{1}^{\prime}\right|_{Y \times T} \otimes L & \stackrel{f^{\prime}}{\longrightarrow} & \left.\tilde{E}_{2}^{\prime}\right|_{Y \times T} \otimes L .
\end{array}
$$

(*) For any $t \in T, E_{1}(t)$ and $\tilde{E}_{2}(t)$ are of pure dimension $d, \chi\left(E_{1}(t)(m)\right)$ $=H_{1}(m), \chi\left(\tilde{E}_{2}(t)(m)\right)=H_{2}(m), \chi\left(E_{1}(t)(-Y)(m)\right)=H_{1}^{\prime}(m)$, $\chi\left(\tilde{E}_{2}(t)(-Y)(m)\right)=H_{2}^{\prime}(m)$ and $\operatorname{ker} \varphi_{f(t)}$ is a stable sheaf on $X \times k(t)$, where $\varphi_{f(t)}:\left.E_{1}(t) \oplus \tilde{E}_{2}(t) \rightarrow \tilde{E}_{2}(t)\right|_{Y \times k(t)}$ is the homomorphism defined by $\varphi_{f(t)}(a, b):=f(t)\left(\left.a\right|_{Y}\right)-\left.b\right|_{Y}$.

We can similarly define a functor $\mathcal{M}_{X, H_{1}^{\prime}, H_{2}^{\prime}}^{(2), H_{1}, H_{2}}$ :

$$
\begin{aligned}
& \mathcal{M}_{X, H_{1}^{\prime}, H_{2}^{\prime}}^{(2), H_{1}, H_{2}}(T):= \\
& \left\{\begin{array}{l|l}
\left(\tilde{E}_{1}, E_{2}, f\right) & \begin{array}{l}
E_{2} \text { is a } T \text {-flat coherent } \mathcal{O}_{X_{2} \times T^{-}} \text {-module, } \\
\tilde{E}_{1} \text { is a } T \text {-flat coherent } \mathcal{O}_{X_{1} \times T^{-} \text {-module }} \\
\text { and } f:\left.\left.E_{2}\right|_{Y \times T} \rightarrow \tilde{E}_{1}\right|_{Y \times T} \text { is a homo- } \\
\text { morphism with the property }\left(*^{\prime}\right)
\end{array}
\end{array}\right\} / \sim
\end{aligned}
$$

where $\sim$ is the equivalence relation defined similarly to that of $\mathcal{M}_{X, H_{1}^{\prime}, H_{2}^{\prime}}^{(1), H_{1}, H_{2}}$.

$\left(*^{\prime}\right)$ For any $t \in T, E_{2}(t)$ and $\tilde{E}_{1}(t)$ are of pure dimension $d, \chi\left(E_{2}(t)(m)\right)$ $=H_{2}(m), \chi\left(\tilde{E}_{1}(t)(m)\right)=H_{1}(m), \chi\left(E_{2}(t)(-Y)(m)\right)=H_{2}^{\prime}(m)$, $\chi\left(\tilde{E}_{1}(t)(-Y)(m)\right)=H_{1}^{\prime}(m)$ and $\operatorname{ker} \varphi_{f(t)}$ is a stable sheaf on $X \times k(t)$, where $\varphi_{f(t)}:\left.E_{2}(t) \oplus \tilde{E}_{1}(t) \rightarrow \tilde{E}_{1}(t)\right|_{Y \times k(t)}$ is the homomorphism defined by $\varphi_{f(t)}(a, b):=f(t)\left(\left.a\right|_{Y}\right)-\left.b\right|_{Y}$. 
Let $\left(E_{1}, \tilde{E}_{2}, f\right)$ be an element of $\mathcal{M}_{X, H_{1}^{\prime}, H_{2}^{\prime}}^{(1), H_{1}, H_{2}}(T)$. If we put

$$
\varphi_{f}:\left.E_{1} \oplus \tilde{E}_{2} \longrightarrow \tilde{E}_{2}\right|_{Y \times T} ; \quad \varphi_{f}(a, b):=f\left(\left.a\right|_{Y \times T}\right)-\left.b\right|_{Y \times T},
$$

then $\operatorname{ker} \varphi_{f}$ is an element of $\mathcal{M}_{X}^{H}(T)$. So we can define a morphism of functors:

$$
\Phi: \mathcal{M}_{X, H_{1}^{\prime}, H_{2}^{\prime}}^{(1), H_{1}, H_{2}} \longrightarrow \mathcal{M}_{X}^{H} ; \quad\left(E_{1}, \tilde{E}_{2}, f\right) \mapsto \operatorname{ker} \varphi_{f}
$$

Proposition 1.8. The morphism $\Phi: \mathcal{M}_{X, H_{1}^{\prime}, H_{2}^{\prime}}^{(1), H_{1}, H_{2}} \rightarrow \mathcal{M}_{X}^{H}$ is a monomorphism. Moreover for any noetherian scheme $S$ over $k$ and for any morphism of functors $h_{S} \rightarrow \mathcal{M}_{X}^{H}, h_{S} \times_{\mathcal{M}_{X}^{H}} \mathcal{M}_{X, H_{1}^{\prime}, H_{2}^{\prime}}^{(1), H_{1}, H_{2}}$ is representable by a subscheme $S_{0}$ of $S$.

Proof. First we will prove that $\Phi$ is a monomorphism. Take $T$-valued points $\left(E_{1}, \tilde{E}_{2}, f\right),\left(E_{1}^{\prime}, \tilde{E}_{2}^{\prime}, f^{\prime}\right)$ of $\mathcal{M}_{X, H_{1}^{\prime} H_{2}^{\prime}}^{(1), H_{1}, H_{2}}$ such that $\Phi\left(\left(E_{1}, \tilde{E}_{2}, f\right)\right)=$ $\Phi\left(\left(E_{1}^{\prime}, \tilde{E}_{2}^{\prime}, f^{\prime}\right)\right)$. Let $\varphi_{f}:\left.E_{1} \oplus \tilde{E}_{2} \rightarrow \tilde{E}_{2}\right|_{Y \times T}$ and $\varphi_{f^{\prime}}:\left.E_{1}^{\prime} \oplus \tilde{E}_{2}^{\prime} \rightarrow \tilde{E}_{2}^{\prime}\right|_{Y \times T}$ be the homomorphisms induced by $f$ and $f^{\prime}$ respectively. Put $E:=\operatorname{ker} \varphi_{f}$ and $E^{\prime}:=\operatorname{ker} \varphi_{f^{\prime}}$. Then there exist a line bundle $\mathrm{L}$ on $T$ and an isomorphism $\sigma: E \stackrel{\sim}{\rightarrow} E^{\prime} \otimes_{\mathcal{O}_{T}} L$. The injection

$$
\begin{array}{ccc}
E_{1}(-Y) & \hookrightarrow & E_{1} \oplus \tilde{E}_{2} \\
a & \mapsto & (a, 0)
\end{array}
$$

factors through $E$ :

$$
E_{1}(-Y) \hookrightarrow E \hookrightarrow E_{1} \oplus \tilde{E}_{2}
$$

On the other hand, the canonical commutative diagram

$$
\begin{array}{ccc}
I_{X_{2}} \otimes E & \longrightarrow & E \\
\downarrow & & \downarrow \\
I_{X_{2}} \otimes\left(E_{1} \oplus \tilde{E}_{2}\right) & \longrightarrow & E_{1} \oplus \tilde{E}_{2}
\end{array}
$$

induces the following commutative diagram:

$$
\begin{array}{ccc}
I_{X_{2}} \otimes E & \longrightarrow & E \\
\downarrow & \nearrow & \downarrow \\
E_{1}(-Y) & \longrightarrow & E_{1} \oplus \tilde{E}_{2} .
\end{array}
$$

The homomorphism $I_{X_{2}} \otimes E \rightarrow E_{1}(-Y)$ is surjective since the canonical homomorphism $E \rightarrow E_{1}$ is surjective. Hence the sequence

$$
0 \longrightarrow E_{1}(-Y) \longrightarrow E \longrightarrow E \otimes \mathcal{O}_{X_{2}} \longrightarrow 0
$$


is exact. The injection

$$
\begin{array}{ccc}
\tilde{E}_{2}(-Y) & \hookrightarrow & E_{1} \oplus \tilde{E}_{2} \\
b & \mapsto & (0, b)
\end{array}
$$

factors through $E$ :

$$
\tilde{E}_{2}(-Y) \hookrightarrow E \hookrightarrow E_{1} \oplus \tilde{E}_{2}
$$

We can easily check that

$$
0 \longrightarrow \tilde{E}_{2}(-Y) \longrightarrow E \longrightarrow E_{1} \longrightarrow 0
$$

is an exact sequence. Similarly to (4) and (6), there are exact sequences:

$$
\begin{gathered}
0 \longrightarrow E_{1}^{\prime}(-Y) \longrightarrow E^{\prime} \longrightarrow E^{\prime} \otimes \mathcal{O}_{X_{2}} \longrightarrow 0 \\
0 \longrightarrow \tilde{E}_{2}^{\prime}(-Y) \longrightarrow E^{\prime} \longrightarrow E_{1}^{\prime} \longrightarrow 0 .
\end{gathered}
$$

From (4) and (7), there is an isomorphism $\sigma_{1}^{\prime}: E_{1}(-Y) \stackrel{\sim}{\rightarrow} E_{1}^{\prime}(-Y) \otimes L$ such that the following diagram commutes:

$$
\begin{array}{ccccc}
I_{X_{2}} \otimes E & \longrightarrow & E_{1}(-Y) & \hookrightarrow & E \\
1 \otimes \sigma \downarrow 2 & & \sigma_{1}^{\prime} \downarrow 2 & & \sigma \downarrow l \\
I_{X_{2}} \otimes E^{\prime} \otimes L & \longrightarrow & E_{1}^{\prime}(-Y) \otimes L & \hookrightarrow & E^{\prime} \otimes L .
\end{array}
$$

Let $\sigma_{1}: E_{1} \stackrel{\sim}{\rightarrow} E_{1}^{\prime} \otimes L$ be the isomorphism obtained by tensoring $\mathcal{O}_{X_{1}}(Y)$ to $\sigma_{1}^{\prime}$. Then we have the following commutative diagram:

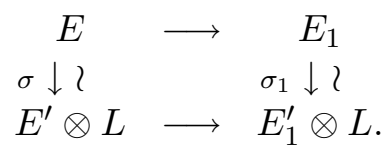

Taking the kernels of the horizontal homomorphisms of the diagram (10) and using (6),(8), we get an isomorphism $\sigma_{2}^{\prime}: \tilde{E}_{2}(-Y) \stackrel{\sim}{\rightarrow} \tilde{E}_{2}^{\prime}(-Y) \otimes L$ such that the diagram

$$
\begin{array}{ccccc}
I_{X_{1}} \otimes E & \longrightarrow & \tilde{E}_{2}(-Y) & \longrightarrow & E \\
1 \otimes \sigma \downarrow 2 & & \sigma_{2}^{\prime} \downarrow \text { ? } & & \sigma \downarrow l \\
I_{X_{1}} \otimes E^{\prime} \otimes L & \longrightarrow & \tilde{E}_{2}^{\prime}(-Y) \otimes L & \longrightarrow & E^{\prime} \otimes L
\end{array}
$$

commutes. Note that there is a commutative diagram

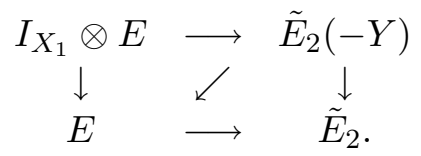


Let $\sigma_{2}: \tilde{E}_{2} \stackrel{\sim}{\longrightarrow} \tilde{E}_{2}^{\prime} \otimes L$ be the isomorphism obtained by tensoring $\mathcal{O}_{X_{2}}(Y)$ to $\sigma_{2}^{\prime}$. Then a commutative diagram

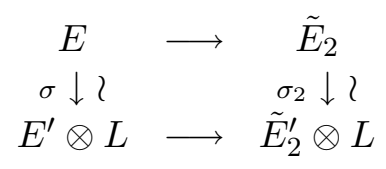

is obtained. From the definition of $E$ and $E^{\prime}$, the diagrams
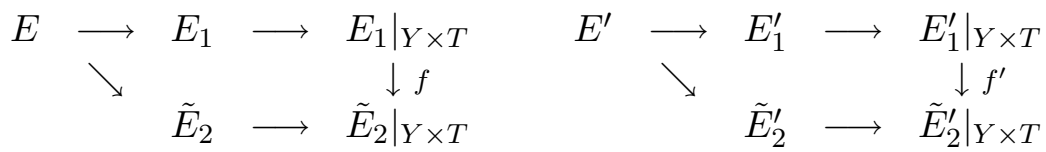

both commute. Hence from (10) and (12), the following commutative diagram is obtained:

$$
\begin{array}{ccc}
\left.E_{1}\right|_{Y \times T} & \stackrel{f}{\longrightarrow} & \left.\tilde{E}_{2}\right|_{Y \times T} \\
\left.\sigma_{1}\right|_{Y \times T} \downarrow 2 & & \left.\sigma_{2}\right|_{Y \times T} \downarrow 2 \\
\left.E_{1}^{\prime}\right|_{Y \times T} \otimes L & \stackrel{f^{\prime}}{\longrightarrow} & \left.\tilde{E}_{2}^{\prime}\right|_{Y \times T} \otimes L .
\end{array}
$$

These mean that $\left(E_{1}, \tilde{E}_{2}, f\right) \sim\left(E_{1}^{\prime}, \tilde{E}_{2}^{\prime}, f^{\prime}\right)$ and so $\Phi$ is a monomorphism.

Next we prove the second assertion of the proposition. Let $S$ be a noetherian scheme over $k$ and $\phi: h_{S} \rightarrow \mathcal{M}_{X}^{H}$ a morphism of functors. $\phi$ is given by an element $E \in \mathcal{M}_{X}^{H}(S)$. From the flattening stratification theorem, there exists a subscheme $S_{1}$ of $S$ such that for any $T \in(\mathrm{Sch} / k)$ and any morphism $f: T \rightarrow S, f$ factors through $S_{1}$ if and only if $(1 \times$ $f)^{*}\left(E \otimes \mathcal{O}_{X_{2}}\right)$ is flat over $T$ and $\chi\left(\left(E \otimes \mathcal{O}_{X_{2}}\right) \otimes k(t)(m)\right)=H(m)-H_{1}^{\prime}(m)$ for all $t \in T$. Let $q: E_{S_{1}} \rightarrow E_{S_{1}} \otimes \mathcal{O}_{X_{2}}$ be the canonical surjection. Since ker $q$ is the image of the homomorphism $I_{X_{2}} \otimes E_{S_{1}} \rightarrow E_{S_{1}}$, it is an $\mathcal{O}_{X_{1} \times S_{1}}$ module. If we put $E^{(1)}:=\operatorname{ker} q \otimes \mathcal{O}_{X_{1}}(Y)$, then $E^{(1)}$ becomes an $S_{1}$-flat $\mathcal{O}_{X_{1} \times S_{1}}$-module. The composition

$g:\left.\left.E_{S_{1}} \longrightarrow E\right|_{X_{1} \times S_{1}} \stackrel{\sim}{\rightarrow} I_{X_{2}} \otimes E\right|_{X_{1} \times S_{1}} \otimes \mathcal{O}_{X_{1}}(Y) \longrightarrow \operatorname{ker} q \otimes \mathcal{O}_{X_{1}}(Y) \stackrel{\sim}{\rightarrow} E^{(1)}$

is surjective. Since the diagram

$$
\begin{aligned}
& I_{X_{2}} \otimes E_{S_{1}} \longrightarrow E_{S_{1}} \\
& I_{X_{2}} \cdot E_{S_{1}}=E^{(1)}(-Y) \hookrightarrow E^{(1)}
\end{aligned}
$$

commutes, the composition $E^{(1)}(-Y) \hookrightarrow E_{S_{1}} \stackrel{g}{\rightarrow} E^{(1)}$ is just the canonical injection induced by the inclusion $\mathcal{O}_{X_{1}}(-Y) \hookrightarrow \mathcal{O}_{X_{1}}$. The image of the 
homomorphism $I_{X_{2}} \otimes \operatorname{ker} g \rightarrow E_{S_{1}}$ is contained in $E^{(1)}(-Y)$ and the composition $E^{(1)}(-Y) \rightarrow E_{S_{1}} \stackrel{g}{\rightarrow} E^{(1)}$ is injective. Hence $I_{X_{2}}$ ker $g=0$ and so ker $g$ is an $\mathcal{O}_{X_{2} \times S_{1}}$-module. Put $\tilde{E}^{(2)}:=\operatorname{ker} g \otimes \mathcal{O}_{X_{2}}(Y)$. Note that $E^{(1)}$ and $\tilde{E}^{(2)}$ are both flat over $S_{1}$. So there exists an open and closed subscheme $S_{0}$ of $S_{1}$ such that a point $s \in S_{1}$ is in $S_{0}$ if and only if the following equalities hold:

$$
\begin{aligned}
& \chi\left(E^{(1)} \otimes k(s)(m)\right)=H_{1}(m), \quad \chi\left(\tilde{E}^{(2)} \otimes k(s)(m)\right)=H_{2}(m) \\
& \chi\left(\tilde{E}^{(2)} \otimes k(s)(-Y)(m)\right)=H_{2}^{\prime}(m) .
\end{aligned}
$$

Since the composite $I_{X_{1}} \otimes E_{S_{1}} \rightarrow E_{S_{1}} \stackrel{g}{\rightarrow} E^{(1)}$ is zero, the image of the canonical homomorphism $I_{X_{1}} \otimes E_{S_{1}} \rightarrow E_{S_{1}}$ is contained in $\tilde{E}^{(2)}(-Y)$. Then we have the following composition:

$$
g^{\prime}:\left.E_{S_{1}} \rightarrow E\right|_{X_{2} \times S_{1}} \stackrel{\sim}{\rightarrow} I_{X_{1}} \otimes E_{S_{1}} \otimes \mathcal{O}_{X_{2}}(Y) \rightarrow \tilde{E}^{(2)}
$$

There exists a homomorphism $\alpha:\left.E_{S_{1}}^{(1)} \rightarrow \tilde{E}^{(2)}\right|_{Y \times S_{1}}$ satisfying the following exact commutative diagram:

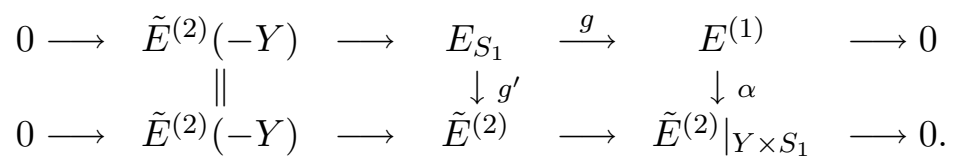

Let $f:\left.\left.E^{(1)}\right|_{Y \times S_{1}} \rightarrow \tilde{E}^{(2)}\right|_{Y \times S_{1}}$ be the homomorphism induced by $\alpha$. Put

$$
\begin{array}{rlc}
\varphi_{f}: \quad E^{(1)} \oplus \tilde{E}^{(2)} & \longrightarrow & \left.\tilde{E}^{(2)}\right|_{Y \times S_{1}} \\
(a, b) & \mapsto f\left(\left.a\right|_{Y \times S_{1}}\right)-\left.b\right|_{Y \times S_{1}} .
\end{array}
$$

Let $i: E_{S_{1}} \rightarrow E^{(1)} \oplus \tilde{E}^{(2)}$ be the homomorphism induced by the homomorphisms $g: E_{S_{1}} \rightarrow E^{(1)}$ and $g^{\prime}: E_{S_{1}} \rightarrow \tilde{E}^{(2)} . \quad i$ is injective since its restriction to every fiber over $S_{1}$ is injective ([4], IV Proposition (11.3.7)). Moreover we can check that the sequence

$$
\left.0 \longrightarrow E_{S_{1}} \stackrel{i}{\longrightarrow} E^{(1)} \oplus \tilde{E}^{(2)} \stackrel{\varphi_{f}}{\longrightarrow} \tilde{E}^{(2)}\right|_{Y \times S_{1}} \longrightarrow 0
$$

is exact.

The triple $\left(E_{S_{0}}^{(1)}, \tilde{E}_{S_{0}}^{(2)}, f_{S_{0}}\right)$ defines an element of $\mathcal{M}_{X, H_{1}^{\prime}, H_{2}^{\prime}}^{(1), H_{1}, H_{2}}\left(S_{0}\right)$. Then we have a morphism $\phi^{\prime}: h_{S_{0}} \rightarrow \mathcal{M}_{X, H_{1}^{\prime}, H_{2}^{\prime}}^{(1), H_{1}, H_{2}}$. From the exactness of (19), the following diagram commutes:

$$
\begin{array}{cccc}
h_{S_{0}} & \hookrightarrow & h_{S} \\
\phi^{\prime} \downarrow & & \downarrow \phi \\
\mathcal{M}_{X, H_{1}^{\prime}, H_{2}^{\prime}}^{(1), H_{1}, H_{2}} & \hookrightarrow & \mathcal{M}_{X}^{H} .
\end{array}
$$


In particular we have a monomorphism

$$
h_{S_{0}} \hookrightarrow \mathcal{M}_{X, H_{1}^{\prime}, H_{2}^{\prime}}^{(1), H_{1}, H_{2}} \times \mathcal{M}_{X}^{H} h_{S} \hookrightarrow h_{S} .
$$

Let $\left(\left(E_{1}, \tilde{E}_{2}, f\right), \psi\right)$ be any element of $\mathcal{M}_{X, H_{1}^{\prime}, H_{2}^{\prime}}^{(1), H_{1}, H_{2}}(T) \times_{\mathcal{M}_{X}^{H}(T)} S(T)$. Put

$$
\varphi_{f}:\left.E_{1} \oplus \tilde{E}_{2} \longrightarrow \tilde{E}_{2}\right|_{Y \times T} ; \quad \varphi_{f}(a, b):=f\left(\left.a\right|_{Y \times T}\right)-\left.b\right|_{Y \times T} .
$$

Then we have $E_{T} \cong \operatorname{ker} \varphi_{f} \otimes L$ for some line bundle $L$ on $T$. Replacing $\left(E_{1}, \tilde{E}_{2}, f\right)$ by $\left(E_{1}, \tilde{E}_{2}, f\right) \otimes L$, we may assume that $E_{T} \cong \operatorname{ker} \varphi_{f}$.

From (4),(6), there are exact sequences:

$$
\begin{gathered}
\left.0 \longrightarrow E_{1}(-Y) \longrightarrow E_{T} \longrightarrow E\right|_{X_{2} \times T} \longrightarrow 0 \\
0 \longrightarrow \tilde{E}_{2}(-Y) \longrightarrow E_{T} \longrightarrow E_{1} \longrightarrow 0 .
\end{gathered}
$$

Since $E_{1}(-Y)(t) \rightarrow E(t)$ is injective for all $t \in T,\left.E\right|_{X_{2} \times T}$ is flat over $T$ ([4], IV Proposition 11.3.7) and $\chi\left(\left.E\right|_{X_{2} \times T}(t)(m)\right)=H(m)-H_{1}^{\prime}(m)$ for all $t \in T$. Hence the morphism $\psi: T \rightarrow S$ factors through $S_{0}$. This proves that $h_{S_{0}} \longrightarrow \mathcal{M}_{X, H_{1}^{\prime}, H_{2}^{\prime}}^{(1), H_{1}, H_{2}} \times \mathcal{M}_{X}^{H} h_{S}$ is an isomorphism.

Let us recall the following well-known result. The proof is in [17] or [9].

THEOREM 1.9. (Simpson) There exists a coarse moduli scheme $M_{X}^{H}$ of $\mathcal{M}_{X}^{H}$.

As a corollary of Proposition 1.8, we have the following result.

THEOREM 1.10. There exists a coarse moduli scheme $M_{X, H_{1}^{\prime}, H_{2}^{\prime}}^{(1), H_{1}, H_{2}}$ of $\mathcal{M}_{X, H_{1}^{\prime}, H_{2}^{\prime}}^{(1), H_{1}, H_{2}}$. Moreover $M_{X, H_{1}^{\prime}, H_{2}^{\prime}}^{(1), H_{1}, H_{2}}$ is a subscheme of $M_{X}^{H}$.

Proof. From the arguments in [17] or [9], we can see that the moduli scheme $M_{X}^{H}$ for $\mathcal{M}_{X}^{H}$ is obtained as a quotient of an open subscheme $R$ of a Quot-scheme by an action of $P G L(V)$ for some vector space $V$ over $k$. Moreover $R$ is a principal $P G L(V)$-bundle over $M_{X}^{H}$. Note that the notion of $e$-stable sheaf is now needless because the boundedness of semistable sheaves has been proven $([7])$. From Proposition 1.8, there exists a subscheme $R^{\prime}$ of $R$ such that $h_{R^{\prime}} \cong h_{R} \times_{\mathcal{M}_{X}^{H}} \mathcal{M}_{X, H_{1}^{\prime}, H_{2}^{\prime}}^{(1), H_{1}, H_{2}}$. From the construction, $R^{\prime} \hookrightarrow R$ is $P G L(V)$-equivariant and $R^{\prime}$ descends to a subscheme $M_{X, H_{1}^{\prime}, H_{2}^{\prime}}^{(1), H_{1}, H_{2}}$ of $M_{X}^{H}$. Since $R^{\prime} \rightarrow M_{X, H_{1}^{\prime}, H_{2}^{\prime}}^{(1), H_{1}, H_{2}}$ is a principle $P G L(V)$-bundle, we can easily see that $M_{X, H_{1}^{\prime}, H_{2}^{\prime}}^{(1), H_{1}, H_{2}}$ is a coarse moduli scheme of $\mathcal{M}_{X, H_{1}^{\prime}, H_{2}^{\prime}}^{(1), H_{1}, H_{2}}$. 
The following Proposition means that $M_{X, H_{1}^{\prime}, H_{2}^{\prime}}^{(1), H_{1}, H_{2}}$ and $M_{X}^{H}$ have the same scheme structure at "general points" of $M_{X, H_{1}^{\prime}, H_{2}^{\prime}}^{(1), H_{1}, H_{2}}$.

Proposition 1.11. Let $R$ be a noetherian local ring and $s$ be the closed point of $\operatorname{Spec} R$. Let $E$ be an $R$-valued point of $\mathcal{M}_{X}^{H}$. Assume that the triple $\left(F_{1}, \tilde{F}_{2}, f\right)$ corresponding to $E(s)$ is contained in $\mathcal{M}_{X, H_{1}^{\prime}, H_{2}^{\prime}}^{(1), H_{1}, H_{2}}(k(s))$ and that $f:\left.\left.F_{1}\right|_{Y \times k(s)} \rightarrow \tilde{F}_{2}\right|_{Y \times k(s)}$ is surjective. Then $E$ is contained in $\mathcal{M}_{X, H_{1}^{\prime}, H_{2}^{\prime}}^{(1), H_{1}, H_{2}}(R)$.

Proof. The canonical homomorphism $E(s) \rightarrow \tilde{F}_{2}$ is surjective since $f$ is surjective. Hence $\tilde{F}_{2}(-Y) \subset E(s)$ is the image of the canonical homomorphism $I_{X_{1}} \otimes E(s) \rightarrow E(s)$. On the other hand there is an exact sequence;

$$
0 \longrightarrow \tilde{F}_{2}(-Y) \longrightarrow E(s) \longrightarrow F_{1} \longrightarrow 0 \text {. }
$$

So we have an isomorphism $E(s) \otimes \mathcal{O}_{X_{1}} \stackrel{\sim}{\rightarrow} F_{1}$. Hence the canonical homomorphism $I_{X_{2}} \otimes E(s) \stackrel{\sim}{\rightarrow} F_{1}(-Y) \rightarrow E(s)$ is injective. Since $E$ is flat over $S, I_{X_{2}} \otimes E \rightarrow E$ is injective and $E \otimes \mathcal{O}_{X_{2}}$ is flat over $S$ ([4], IV Proposition (11.3.7)). From the proof of Proposition 1.8, $E$ is contained in $\mathcal{M}_{X, H_{1}^{\prime}, H_{2}^{\prime}}^{(1), H_{1}, H_{2}}(R)$.

Remark 1.12. Take a member $E \in M_{X}^{H}(k)$. Let $\left(E^{(1)}, \tilde{E}^{(2)}, f_{E}\right) \in$ $M_{X, H_{1}^{\prime}, H_{2}^{\prime}}^{(1), H_{1}, H_{2}}(k)$ and $\left(\tilde{E}^{(1)}, E^{(2)}, g_{E}\right) \in M_{X, G_{1}^{\prime}, G_{2}^{\prime}}^{(2), G_{1}, G_{2}}(k)$ be the corresponding triples. Then we can check that $f_{E}:\left.\left.E^{(1)}\right|_{Y} \rightarrow \tilde{E}^{(2)}\right|_{Y}$ is surjective if and only if $g_{E}:\left.\left.E^{(2)}\right|_{Y} \rightarrow \tilde{E}^{(1)}\right|_{Y}$ is injective. If a point $p=[E] \in M_{X}^{H}(k)$ satisfies this equivalence condition, then we can see from the proof of Proposition 1.11 that $M_{X, G_{1}^{\prime}, G_{2}^{\prime}}^{(2), G_{1}, G_{2}}$ and $M_{X}^{H}$ have the same scheme structure at $p$.

The following proposition means that we can fix the rank of the sheaf restricted to $X_{1}$ or $X_{2}$ in considering the moduli space of stable sheaves on $X$.

Proposition 1.13. Let $S$ be a connected locally noetherian scheme and $E$ be an element of $\mathcal{M}_{X}^{H}(S)$. Then $a_{0}\left(\left.E\right|_{X_{1} \times S}(s)\right)$ and $a_{0}\left(\left.E\right|_{X_{1} \times S}(s)\right)$ are constant on $S$, where $a_{0}\left(\left.E\right|_{X_{i} \times S}(s)\right)$ is the integer defined before Definition 1.6. 
Proof. Take any point $s$ of $S$. Let $t_{1}, \ldots, t_{d} \in H^{0}\left(X, \mathcal{O}_{X}(1)\right)$ be general members such that $Z_{1} \cap \cdots \cap Z_{d} \cap Y=\emptyset$ and $\left.E\right|_{\left(Z_{1} \cap \cdots \cap Z_{i-1}\right) \times S}(s) \otimes \mathcal{O}_{X}(-1) \stackrel{t_{i}}{\rightarrow}$ $\left.E\right|_{\left(Z_{1} \cap \cdots \cap Z_{i-1}\right) \times S}(s)$ are injective for $i=1, \ldots, d$, where $Z_{i}:=Z\left(t_{i}\right)$ is the zero scheme of $t_{i}$. Then each $\left.E\right|_{\left(Z_{1} \cap \cdots \cap Z_{i}\right) \times S}$ is flat over a neighborhood of $s$. In particular $\left.E\right|_{\left(Z_{1} \cap \cdots \cap Z_{d}\right) \times S}$ is finite and flat over a neighborhood of $s$. Put $W_{1}:=\left(Z_{1} \cap \cdots \cap Z_{d} \cap X_{1}\right) \times S$ and $W_{2}:=\left(Z_{1} \cap \cdots \cap Z_{d} \cap X_{2}\right) \times$ $S$. Then $\left.E\right|_{W_{1}}$ and $\left.E\right|_{W_{2}}$ are both flat over a neighborhood of $s$. Hence $a_{0}\left(\left.E\right|_{X_{1} \times S}(t)\right)=\chi\left(\left.E\right|_{W_{1}}(t)\right)$ and $a_{0}\left(\left.E\right|_{X_{2} \times S}(t)\right)=\chi\left(\left.E\right|_{W_{2}}(t)\right)$ are constant on a neighborhood of $s$.

\section{§2. Direct construction of the moduli space of triples}

In this section we will give another construction of the moduli space $M_{X, H_{1}^{\prime}, H_{2}^{\prime}}^{(1), H_{1}, H_{2}}$ of triples. There exists an integer $m_{0}$ such that for any integer $m \geq m_{0}$ and for any geometric point $\left(E_{1}, \tilde{E}_{2}, f\right) \in \mathcal{M}_{X, H_{1}^{\prime}, H_{2}^{\prime}}^{(1), H_{1}, H_{2}}(k)$,

(i) $H^{0}\left(E_{1}(m)\right) \otimes \mathcal{O}_{X_{1}} \rightarrow E_{1}(m)$ and $H^{0}\left(\tilde{E}_{2}(m)\right) \otimes \mathcal{O}_{X_{2}} \rightarrow \tilde{E}_{2}(m)$ are surjective, and

(ii) $H^{i}\left(E_{1}(m)\right)=0, H^{i}\left(\tilde{E}_{2}(m)\right)=0$ and $H^{i}\left(\tilde{E}_{2}(-Y)(m)\right)=0$ for $i>0$.

Let $V_{1}$ (resp. $V_{2}$ ) be a vector space over $k$ of dimension $H_{1}\left(m_{0}\right)$ (resp. $\left.H_{2}\left(m_{0}\right)\right)$. Let us consider the open subschemes

$$
\begin{aligned}
& Q_{1}:= \\
& \left\{\begin{array}{l|l}
{\left[V_{1} \otimes \mathcal{O}_{X_{1}} \rightarrow E_{1}\right] \in \operatorname{Quot}_{V_{1} \otimes \mathcal{O}_{X_{1}} / X_{1} / k}^{H_{1}\left(x+m_{0}\right)}} & \begin{array}{l}
V_{1} \rightarrow H^{0}\left(E_{1}\right) \text { is bijective, } \\
E_{1} \text { is of pure dimension } d \\
\text { and } H^{i}\left(E_{1}\right)=0 \text { for all } i>0
\end{array}
\end{array}\right\}, \\
& Q_{2}:= \\
& \left\{\begin{array}{l|l}
{\left[V_{2} \otimes \mathcal{O}_{X_{2}} \rightarrow \tilde{E}_{2}\right] \in \operatorname{Quot}_{V_{2} \otimes \mathcal{O}_{X_{2}} / X_{2} / k}^{H_{2}\left(x+m_{0}\right)}} & \begin{array}{l}
V_{2} \rightarrow H^{0}\left(\tilde{E}_{2}\right) \text { is bijective, } \\
\tilde{E}_{2} \text { is of pure dimension } d \\
\text { and } H^{i}\left(\tilde{E}_{2}\right)=0 \text { for all } i>0
\end{array}
\end{array}\right\}
\end{aligned}
$$

of Quot ${ }_{V_{1} \otimes \mathcal{O}_{X_{1}} / X_{1} / k}^{H_{1}\left(x+m_{0}\right)}$ and Quot $\operatorname{Qut}_{V_{2} \otimes \mathcal{O}_{X_{2}} / X_{2} / k}^{H_{2}\left(x+m_{0}\right)}$ respectively. Let $V_{1} \otimes \mathcal{O}_{X_{1} \times Q_{1} \times Q_{2}}$ $\rightarrow \mathcal{E}_{1}$ and $V_{2} \otimes \mathcal{O}_{X_{2} \times Q_{1} \times Q_{2}} \rightarrow \tilde{\mathcal{E}}_{2}$ be the pull back of the universal quotient sheaves. Then $\left.\mathcal{E}_{1}\right|_{Y \times Q_{1} \times Q_{2}}$ and $\left.\tilde{\mathcal{E}}_{2}\right|_{Y \times Q_{1} \times Q_{2}}$ are flat over $Q_{1} \times Q_{2}$. From 
the result of base change theorem $([1],(1.1))$, there exists a coherent sheaf $\mathcal{H}$ on $Q_{1} \times Q_{2}$ such that

$$
\mathcal{H o m}\left(\mathcal{H} \otimes \mathcal{O}_{T}, M\right) \stackrel{\sim}{\longrightarrow}\left(\pi_{T}\right)_{*}\left(\mathcal{H o m}\left(\mathcal{E}_{1} \otimes \mathcal{O}_{Y \times T}, \tilde{\mathcal{E}}_{2} \otimes \mathcal{O}_{Y \times T} \otimes M\right)\right)
$$

for any morphism $T \rightarrow Q_{1} \times Q_{2}$ and any quasi-coherent sheaf $M$ on $T$, where $\pi_{T}: Y \times T \rightarrow T$ is the projection. Let us consider $\mathbf{V}(\mathcal{H})=\operatorname{Spec}(S(\mathcal{H}))$. From the property of $\mathcal{H}$, the canonical homomorphism $\mathcal{H} \otimes \mathcal{O}_{\mathbf{V}(\mathcal{H})} \rightarrow \mathcal{O}_{\mathbf{V}(\mathcal{H})}$ corresponds to a homomorphism:

$$
\tilde{f}: \mathcal{E}_{1} \otimes \mathcal{O}_{Y \times \mathbf{V}(\mathcal{H})} \longrightarrow \tilde{\mathcal{E}}_{2} \otimes \mathcal{O}_{Y \times \mathbf{V}(\mathcal{H})}
$$

Then we can define the following homomorphism;

$$
\begin{aligned}
& \varphi_{\tilde{f}}:\left(\mathcal{E}_{1}\right)_{\mathbf{V}(\mathcal{H})} \oplus\left(\tilde{\mathcal{E}}_{2}\right)_{\mathbf{V}(\mathcal{H})} \longrightarrow \tilde{\mathcal{E}}_{2} \otimes \mathcal{O}_{Y \times \mathbf{V}(\mathcal{H})} \\
&(a, b) \mapsto \tilde{f}\left(\left.a\right|_{Y \times \mathbf{V}(\mathcal{H})}\right)-\left.b\right|_{Y \times \mathbf{V}(\mathcal{H})}
\end{aligned}
$$

If we put $\mathcal{E}^{\prime}:=\operatorname{ker} \varphi_{\tilde{f}} \otimes \mathcal{O}_{X}\left(-m_{0}\right)$, then $\mathcal{E}^{\prime}$ is flat over $\mathbf{V}(\mathcal{H})$. We put

$$
P:=\left\{\begin{array}{l|l}
s \in \mathbf{V}(\mathcal{H}) & \begin{array}{l}
\mathcal{E}^{\prime}(s) \text { is a stable sheaf on } X \times k(s) \\
\chi\left(\mathcal{E}_{1}(s)(-Y)(m)\right)=H_{1}^{\prime}\left(m+m_{0}\right) \text { and } \\
\chi\left(\tilde{\mathcal{E}}_{2}(s)(-Y)(m)\right)=H_{2}^{\prime}\left(m+m_{0}\right)
\end{array}
\end{array}\right\} .
$$

If we put $\mathcal{E}:=\left.\mathcal{E}^{\prime}\right|_{X \times P}$, then it induces a morphism

$$
\Pi^{\prime}: h_{P} \longrightarrow \mathcal{M}_{X, H_{1}^{\prime}, H_{2}^{\prime}}^{(1), H_{1}, H_{2}}
$$

Let $\Pi: P \rightarrow M_{X, H_{1}^{\prime}, H_{2}^{\prime}}^{(1), H_{1}, H_{2}}$ be the morphism induced by the composition

$$
h_{P} \stackrel{\Pi^{\prime}}{\rightarrow} \mathcal{M}_{X, H_{1}^{\prime}, H_{2}^{\prime}}^{(1), H_{1}, H_{2}} \rightarrow h_{M_{X, H_{1}^{\prime}, H_{2}^{\prime}}^{(1), H_{1}, H_{2}}} .
$$

Put $G:=\left(G L\left(V_{1}\right) \times G L\left(V_{2}\right)\right) / \mathbf{G}_{m}$, where $\mathbf{G}_{m} \hookrightarrow G L\left(V_{1}\right) \times G L\left(V_{2}\right)$ is the diagonal embedding.

Theorem 2.1. $\Pi: P \longrightarrow M_{X, H_{1}^{\prime}, H_{2}^{\prime}}^{(1), H_{1}, H_{2}}$ is a principal $G$-bundle.

Proof. Let $S$ be a locally noetherian scheme over $k$ and take elements $g \in G$ and $x \in \mathbf{V}(\mathcal{H})(S) . g$ is given by a line bundle $\mathcal{L}$ on $S$ and two isomorphisms $g_{i}: V_{i} \otimes \mathcal{O}_{S} \stackrel{\sim}{\rightarrow} V_{i} \otimes \mathcal{O}_{S} \otimes \mathcal{L}(i=1,2) . x$ is determined by 
quotients $p_{1}: V_{1} \otimes \mathcal{O}_{X_{1} \times S} \rightarrow E_{1}, p_{2}: V_{2} \otimes \mathcal{O}_{X_{2} \times S} \rightarrow \tilde{E}_{2}$, and a homomorphism $f:\left.\left.E_{1}\right|_{Y \times S} \rightarrow \tilde{E}_{2}\right|_{Y \times S}$. Let $g \cdot x$ be the $S$-valued point of $\mathbf{V}(\mathcal{H})$ determined by the following data:

$$
\begin{aligned}
& V_{1} \otimes \mathcal{O}_{X_{1} \times S} \stackrel{g_{1}}{\longrightarrow} V_{1} \otimes \mathcal{O}_{X_{1} \times S} \otimes \mathcal{L} \stackrel{p_{1}}{\longrightarrow} E_{1} \otimes \mathcal{L}, \\
& V_{2} \otimes \mathcal{O}_{X_{2} \times S} \stackrel{g_{2}}{\longrightarrow} V_{2} \otimes \mathcal{O}_{X_{1} \times S} \otimes \mathcal{L} \stackrel{p_{2}}{\longrightarrow} \tilde{E}_{2} \otimes \mathcal{L} \quad \text { and } \\
& \left.\left.E_{1} \otimes \mathcal{L}\right|_{Y \times S} \stackrel{f \otimes 1}{\longrightarrow} \tilde{E}_{2} \otimes \mathcal{L}\right|_{Y \times S} .
\end{aligned}
$$

Then we can define the action:

$$
\sigma: G \times \mathbf{V}(\mathcal{H}) \rightarrow \mathbf{V}(\mathcal{H}) ; \quad(g, x) \mapsto g \cdot x
$$

$\sigma$ induces the action of $G$ on $P$ and the morphism $\Pi^{\prime}: h_{P} \rightarrow \mathcal{M}_{X, H_{1}^{\prime}, H_{2}^{\prime}}^{(1), H_{1}, H_{2}}$ is $G$-equivariant. So we obtain a morphism of functors $\psi: h_{P} / h_{G} \rightarrow$ $\mathcal{M}_{X, H_{1}^{\prime}, H_{2}^{\prime}}^{(1), H_{1}, H_{2}}$, where $h_{P} / h_{G}$ is the functor defined by

$$
\left(h_{P} / h_{G}\right)(S):=P(S) / G(S)
$$

for any locally noetherian scheme $S$ over $k$. Let $x$ and $x^{\prime}$ be $S$-valued points of $P$ such that $\Pi^{\prime}(x)=\Pi^{\prime}\left(x^{\prime}\right)$. Let $p_{1}: V_{1} \otimes \mathcal{O}_{X_{1} \times S} \rightarrow E_{1}$ and $p_{2}: V_{2} \otimes \mathcal{O}_{X_{2} \times S} \rightarrow \tilde{E}_{2}$ be the quotient sheaves determined by $x$ and $p_{1}^{\prime}$ : $V_{1} \otimes \mathcal{O}_{X_{1} \times S} \rightarrow E_{1}^{\prime}$ and $p_{2}^{\prime}: V_{2} \otimes \mathcal{O}_{X_{2} \times S} \rightarrow \tilde{E}_{2}^{\prime}$ be the quotient sheaves determined by $x^{\prime}$. Let $f:\left.\left.E_{1}\right|_{Y \times S} \rightarrow \tilde{E}_{2}\right|_{Y \times S}$ and $f^{\prime}:\left.\left.E_{1}^{\prime}\right|_{Y \times S} \rightarrow \tilde{E}_{2}^{\prime}\right|_{Y \times S}$ be the homomorphisms determined by $x$ and $x^{\prime}$ respectively. Then there exists a line bundle $\mathcal{L}$ on $S$ such that $E_{1} \cong E_{1}^{\prime} \otimes \mathcal{L}, \tilde{E}_{2} \cong \tilde{E}_{2}^{\prime} \otimes \mathcal{L}$ and the following diagram commutes;

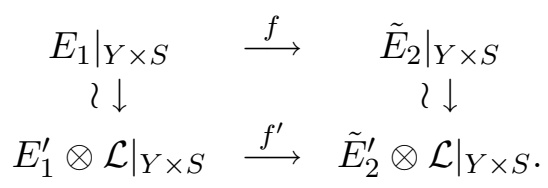

So we have an isomorphism $g_{1}: V_{1} \otimes \mathcal{O}_{S} \stackrel{\sim}{\rightarrow} \pi_{*}\left(E_{1}\right) \stackrel{\sim}{\rightarrow} \pi_{*}\left(E_{1}^{\prime} \otimes \mathcal{L}\right) \stackrel{\sim}{\rightarrow} V_{1} \otimes \mathcal{L}$, where $\pi: X_{1} \times S \rightarrow S$ is the projection. The isomorphism $\tilde{E}_{2} \cong \tilde{E}_{2}^{\prime} \otimes \mathcal{L}$ induces the isomorphism $g_{2}: V_{2} \otimes \mathcal{O}_{S} \stackrel{\sim}{\rightarrow} V_{2} \otimes \mathcal{L}$. Then $\left[\left(g_{1}, g_{2}\right)\right] \cdot x^{\prime}=x$ and so $\psi$ is a monomorphism. It is easy to see that $\psi(R)$ is surjective for any local ring $R$. Hence the sheaves associated to the presheaves $h_{P} / h_{G}$, $\mathcal{M}_{X, H_{1}^{\prime}, H_{2}^{\prime}}^{(1), H_{1}, H_{2}}$ with respect to Zariski topology are isomorphic. From this fact one sees that the morphism $\Pi^{\prime}: P \rightarrow \mathcal{M}_{X, H_{1}^{\prime}, H_{2}^{\prime}}^{(1), H_{1}, H_{2}}$ is formally smooth and thus the morphism $\Pi: P \rightarrow M_{X, H_{1}^{\prime}, H_{2}^{\prime}}^{(1), H_{1}, H_{2}}$ is smooth. 
Let us consider the morphism

$$
\varphi: G \times P \longrightarrow P \times_{M_{X, H_{1}^{\prime}, H_{2}^{\prime}}^{(1), H_{1}, H_{2}}} P
$$

We must prove that $\varphi$ is an isomorphism. Note that $P \times_{M_{X, H_{1}^{\prime}, H_{2}^{\prime}}^{(1), H_{1}, H_{2}}} P \cong$ $P \times{ }_{\mathcal{M}_{X, H_{1}^{\prime}, H_{2}^{\prime}}^{(1), H_{1}, H_{2}}} P$. First we will prove that $\varphi(R)$ is injective for any artinian local ring $R$ over $k$. Let $(g, x),\left(g^{\prime}, x^{\prime}\right)$ be two elements of $G(R) \times P(R)$ such that $(g \cdot x, x)=\left(g^{\prime} \cdot x^{\prime}, x^{\prime}\right)$. Then $x=x^{\prime}$ and $g^{-1} g^{\prime} x=x$. Let $\left(E_{1}, \tilde{E}_{2}, f\right)$ be the triple determined by $x$ and $E$ be the associated coherent sheaf on $X \times R$. Then we have $\operatorname{Hom}_{X \times R}(E, E) \cong R$. If we write $\left(g_{1}, g_{2}\right):=g^{-1} g^{\prime}$, then there are isomorphisms $h_{1}: E_{1} \stackrel{\sim}{\rightarrow} E_{1}, h_{2}: \tilde{E}_{2} \stackrel{\sim}{\rightarrow} \tilde{E}_{2}$ such that the diagrams

$$
\begin{array}{cccccc}
V_{1} \otimes \mathcal{O}_{X_{1}} & \stackrel{p_{1}}{\longrightarrow} & E_{1} & V_{2} \otimes \mathcal{O}_{X_{2}} & \stackrel{p_{2}}{\longrightarrow} & \tilde{E}_{2} \\
g_{1} \downarrow \prec & & h_{1} \downarrow \prec & g_{2} \downarrow 2 & & h_{2} \downarrow \text { ? } \\
V_{1} \otimes \mathcal{O}_{X_{1}} & \stackrel{p_{1}}{\longrightarrow} & E_{1} & V_{2} \otimes \mathcal{O}_{X_{2}} & \stackrel{p_{2}}{\longrightarrow} & \tilde{E}_{2}
\end{array}
$$

commute, where $p_{1}$ and $p_{2}$ are quotients determined by $x$, and the diagram

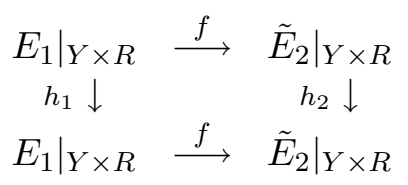

commutes. $\left(h_{1}, h_{2}\right)$ induces the automorphism $E \stackrel{\sim}{\rightarrow} E$. Since $\operatorname{Hom}(E, E)=$ $R$, this isomorphism is a multiplication by an element $c$ of $R$. So $h_{1}=c \cdot 1$ and $h_{2}=c \cdot 1$. Since $V_{1} \otimes R \cong H^{0}\left(E_{1}\right)$ and $V_{2} \otimes R \cong H^{0}\left(\tilde{E}_{2}\right), g_{1}=c \cdot 1$ and $g_{2}=c \cdot 1$. Hence $g^{-1} g^{\prime}=1$ in $G(R)$ and $(g, x)=\left(g^{\prime}, x^{\prime}\right)$.

Hence $\varphi(R)$ is injective for all artinian local rings $R$ over $k$ and so $\varphi$ is a monomorphism. Since $\psi$ is a monomorphism, $\varphi(S)$ is surjective for any $S \in(\mathrm{Sch} / k)$. Hence $\varphi$ is an isomorphism.

Remark 2.2. If $X_{1}, X_{2}$ are non-singular curves, then $\mathcal{H}$ is a locally free sheaf. However, $\mathcal{H}$ is not necessarily locally free in higher dimensional case. (See Remark 5.2 for this example.)

Remark 2.3. By [[9], Proposition 4.10], there exists an integer $l_{0}$ such that for any $l \geq l_{0}$, for any $E \in \mathcal{M}_{X}^{H}(k)$ and for any coherent subsheaf $F$ of $E$ with $0<a_{0}(F)<a_{0}(E)$, the inequality $h^{0}(F(l)) / a_{0}(F)<h^{0}(E(l)) / a_{0}(E)$ 
holds. We assume farther that $m_{0} \geq l_{0}$. Then we can also prove that the quotient $P / G$ exists by using geometric invariant theory. Namely one can show that all points of $P$ are stable points with respect to the action of $G$ and some polarization.

Remark 2.4. Assume that $H_{1} \neq 0$ and $H_{2} \neq 0$. Consider the projective bundle $\mathbf{P}(\mathcal{H})$ over $Q_{1} \times Q_{2}$. Let $\bar{f}: \mathcal{E}_{1} \otimes \mathcal{O}_{Y \times \mathbf{P}(\mathcal{H})} \longrightarrow \tilde{\mathcal{E}}_{2} \otimes \mathcal{O}_{Y \times \mathbf{P}(\mathcal{H})} \otimes$ $\mathcal{O}_{\mathbf{P}(\mathcal{H})}(1)$ be the homomorphism corresponding to the canonical surjection $\mathcal{H} \otimes \mathcal{O}_{\mathbf{P}(\mathcal{H})} \rightarrow \mathcal{O}_{\mathbf{P}(\mathcal{H})}(1)$. Let $\varphi_{\bar{f}}:\left(\mathcal{E}_{1}\right)_{\mathbf{P}(\mathcal{H})} \oplus \tilde{\mathcal{E}}_{2} \otimes \mathcal{O}_{\mathbf{P}(\mathcal{H})}(1) \rightarrow \tilde{\mathcal{E}}_{2} \otimes \mathcal{O}_{\mathbf{P}(\mathcal{H})}(1) \otimes$ $\mathcal{O}_{Y \times \mathbf{V}(\mathcal{H})}$ be the induced homomorphism. Put

$$
\bar{P}:=\left\{\begin{array}{l|l}
x \in \mathbf{P}(\mathcal{H}) & \begin{array}{l}
\operatorname{ker} \varphi_{\bar{f}}(x) \otimes \mathcal{O}_{X}\left(-m_{0}\right) \text { is stable } \\
\chi\left(\mathcal{E}_{1}(s)(-Y)(m)\right)=H_{1}^{\prime}\left(m+m_{0}\right) \text { and } \\
\chi\left(\tilde{\mathcal{E}}_{2}(s)(-Y)(m)\right)=H_{2}^{\prime}\left(m+m_{0}\right)
\end{array}
\end{array}\right\} .
$$

Then $\bar{P}$ is an open subscheme of $\mathbf{P}(\mathcal{H})$. By the same arguments as Theorem 2.1 , we can see that $\bar{P} \rightarrow M_{X, H_{1}^{\prime}, H_{2}^{\prime}}^{(1), H_{1}, H_{2}}$ is a principal $P G L\left(V_{1}\right) \times P G L\left(V_{2}\right)$ bundle.

\section{§3. Parabolic triples}

Let $X$ be a projective scheme over $k$ satisfying the condition $(\dagger)$ of section 1 .

Definition 3.1. A parabolic triple is a triple $\left(\left(E_{1}\right)_{*},\left(E_{2}\right)_{*}, \sigma\right)$, where $\left(E_{i}\right)_{*}$ is a filtration $E_{i}(-Y) \subset E_{i}^{\prime} \subset E_{i}$ of coherent sheaves on $X_{i}$ of pure dimension $d$ for $i=1,2$ and $\sigma$ an isomorphism $\sigma: E_{1} / E_{1}^{\prime} \stackrel{\sim}{\rightarrow} E_{2} / E_{2}^{\prime}$ on $Y$.

Definition 3.2. Two parabolic triples $\left(\left(E_{1}\right)_{*},\left(E_{2}\right)_{*}, \sigma\right)$ and $\left(\left(F_{1}\right)_{*}\right.$, $\left.\left(F_{2}\right)_{*}, \tau\right)$ are said to be isomorphic if there exist isomorphisms $\theta_{i}: E_{i} \stackrel{\sim}{\longrightarrow} F_{i}$ for $i=1,2$ such that $\theta_{i}\left(E_{i}^{\prime}\right)=F_{i}^{\prime}$ for $i=1,2$ and the diagram

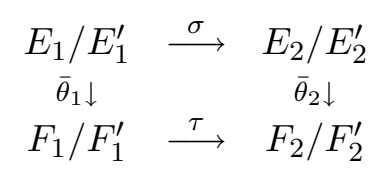

commutes, where $\bar{\theta}_{i}: E_{i} / E_{i}^{\prime} \stackrel{\sim}{\rightarrow} F_{i} / F_{i}^{\prime}$ is the isomorphism induced by $\theta_{i}$.

Proposition 3.3. There exists a bijective correspondence between the isomorphism classes of coherent sheaves of pure dimension $d$ on $X$ and the isomorphism classes of parabolic triples. 
Proof. Let $E$ be a coherent sheaf of pure dimension $d$ on $X$. As in the argument in section 1 , there is an exact sequence

$$
0 \longrightarrow E \longrightarrow E^{(1)} \oplus E^{(2)} \longrightarrow E^{(0)} \longrightarrow 0 .
$$

Let $E_{*}^{(i)}$ be the filtration $E^{(i)}(-Y) \subset \tilde{E}^{(i)}(-Y) \subset E^{(i)}$ for $i=1,2$. Let $\sigma$ be the canonical isomorphism $E^{(1)} / \tilde{E}^{(1)}(-Y) \stackrel{\sim}{\rightarrow} E^{(0)} \stackrel{\sim}{\rightarrow} E^{(2)} / \tilde{E}^{(2)}(-Y)$. Then we obtain a parabolic triple $\left(E_{*}^{(1)}, E_{*}^{(2)}, \sigma\right)$ for $E$. It is easy to see that $E \mapsto\left(E_{*}^{(1)}, E_{*}^{(2)}, \sigma\right)$ is a bijective correspondence.

A flat family of parabolic triples on $X_{T} / T$ is a triple $\left(\left(E_{1}\right)_{*},\left(E_{2}\right)_{*}, \sigma\right)$ such that for each $i,\left(E_{i}\right)_{*}$ is a filtration $E_{i}(-Y) \subset E_{i}^{\prime} \subset E_{i}$ of coherent sheaves on $X_{T}$ such that $E_{i}$ and $E_{i} / E_{i}^{\prime}$ are flat over $T, E_{i}(t)$ is of pure dimension $d$ for any $t \in T$ and $\sigma: E_{1} / E_{1}^{\prime} \stackrel{\sim}{\rightarrow} E_{2} / E_{2}^{\prime}$ is an isomorphism. For a flat family $\left(\left(E_{1}\right)_{*},\left(E_{2}\right)_{*}, \sigma\right)$ of parabolic triples, we define a homomorphism

$$
\varphi_{\sigma}: E_{1} \oplus E_{2} \longrightarrow E_{2} / E_{2}^{\prime}
$$

by $\varphi_{\sigma}(a, b)=\sigma(\bar{a})-\bar{b}$ where $\bar{a}$ is the image of $a$ by $E_{1} \rightarrow E_{1} / E_{1}^{\prime}$. Let $\left(H_{i}\right)_{*}$ be sequences of numerical polynomials $\tilde{H}_{i}(m), H_{i}(m), \tilde{H}_{i}^{\prime}(m), H_{i}^{\prime}(m)$ for $i=1,2$ such that $H(m)=H_{1}(m)+H_{2}^{\prime}(m)=H_{1}^{\prime}(m)+H_{2}(m)$.

Definition 3.4. We define a functor

$$
\operatorname{par}-\mathcal{M}_{X}^{\left(H_{1}\right)_{*},\left(H_{2}\right)_{*}}:(\operatorname{Sch} / k) \longrightarrow(\text { Sets })
$$

by

$$
\begin{aligned}
& \operatorname{par}-\mathcal{M}_{X}^{\left(H_{1}\right)_{*},\left(H_{2}\right)_{*}}(T):= \\
& \left.\qquad \begin{array}{c}
\left(\left(E_{1}\right)_{*},\left(E_{2}\right)_{*}, \sigma\right) ; \text { a flat family of parabolic } \\
\text { triples on } X_{T} / T \text { such that for any } t \in T, \\
\left(\left(E_{1}\right)_{*},\left(E_{2}\right)_{*}, \sigma\right) \otimes k(t) \text { satisfies the } \\
\text { following condition (a) }
\end{array}\right\} / \sim
\end{aligned}
$$

where $\sim$ is the equivalence relation defined by (b).

(a) $\chi\left(E_{i}^{\prime}(t)(Y)(m)\right)=\tilde{H}_{i}(m), \chi\left(E_{i}(t)(m)\right)=H_{i}(m), \chi\left(E_{i}^{\prime}(t)(m)\right)=$ $\tilde{H}_{i}^{\prime}(m)$ and $\chi\left(E_{i}(t)(-Y)(m)\right)=H_{i}^{\prime}(m)$ for $i=1,2$ and $\operatorname{ker} \varphi_{\sigma}(t)$ is a stable sheaf on $X \times k(t)$. 
(b) $\left(\left(E_{1}\right)_{*},\left(E_{2}\right)_{*}, \sigma\right) \sim\left(\left(F_{1}\right)_{*},\left(F_{2}\right)_{*}, \tau\right)$ if there are a line bundle $L$ on $T$ and isomorphisms $\theta_{i}: E_{i} \stackrel{\sim}{\rightarrow} F_{i} \otimes L$ with $\theta_{i}\left(E_{i}^{\prime}\right)=F_{i}^{\prime} \otimes L$ for $i=1,2$ such that the diagram

$$
\begin{array}{ccc}
E_{1} / E_{1}^{\prime} & \stackrel{\bar{\theta}_{1}}{\longrightarrow} & F_{1} / F_{1}^{\prime} \otimes L \\
\sigma \downarrow & & \tau \downarrow \\
E_{2} / E_{2}^{\prime} & \stackrel{\bar{\theta}_{2}}{\longrightarrow} & F_{2} / F_{2}^{\prime} \otimes L
\end{array}
$$

commutes, where $\bar{\theta}_{i}: E_{i} / E_{i}^{\prime} \stackrel{\sim}{\rightarrow} F_{i} / F_{i}^{\prime} \otimes L$ is the isomorphism induced by $\theta_{i}$.

We can define a morphism of functors

$$
\Psi: \operatorname{par}-\mathcal{M}_{X}^{\left(H_{1}\right)_{*},\left(H_{2}\right)_{*}} \longrightarrow \mathcal{M}_{X}^{H}
$$

by $\Psi\left(\left(E_{1}\right)_{*},\left(E_{2}\right)_{*}, \sigma\right):=\operatorname{ker} \varphi_{\sigma}$.

Proposition 3.5. $\Psi$ : par- $\mathcal{M}_{X}^{\left(H_{1}\right)_{*},\left(H_{2}\right)_{*}} \rightarrow \mathcal{M}_{X}^{H}$ is a monomorphism. Moreover par- $\mathcal{M}_{X}^{\left(H_{1}\right)_{*},\left(H_{2}\right)_{*}}=\mathcal{M}_{X, H_{1}^{\prime}, \tilde{H}_{2}^{\prime}}^{(1), H_{1}, \tilde{H}_{2}} \cap \mathcal{M}_{X, \tilde{H}_{1}^{\prime}, H_{2}^{\prime}}^{(2), \tilde{H}_{1}, H_{2}}$ as subfunctors of $\mathcal{M}_{X}^{H}$

Proof. Let $\left(\left(E_{1}\right)_{*},\left(E_{2}\right)_{*}, \sigma\right)$ and $\left(\left(F_{1}\right)_{*},\left(F_{2}\right)_{*}, \tau\right)$ be two $T$-valued points of par- $\mathcal{M}_{X}^{\left(H_{1}\right)_{*},\left(H_{2}\right)_{*}}$ such that $\Psi\left(\left(E_{1}\right)_{*},\left(E_{2}\right)_{*}, \sigma\right)=\Psi\left(\left(F_{1}\right)_{*},\left(F_{2}\right)_{*}, \tau\right)$. If we put $E:=\operatorname{ker} \varphi_{\sigma}$ and $F:=\operatorname{ker} \varphi_{\tau}$, then there exists a line bundle $L$ on $T$ such that $E \cong F \otimes L$. From the similar arguments to the proof of Proposition 1.8, the homomorphism

$$
\begin{array}{ccc}
E_{1}(-Y) & \hookrightarrow & E_{1} \oplus E_{2} \\
a & \mapsto & (a, 0)
\end{array}
$$

factors through $E$ and the induced sequence

$$
0 \longrightarrow E_{1}(-Y) \longrightarrow E \longrightarrow E \otimes \mathcal{O}_{X_{2}} \longrightarrow 0
$$

is exact. Similarly the exact sequence

$$
0 \longrightarrow F_{1}(-Y) \longrightarrow F \longrightarrow F \otimes \mathcal{O}_{X_{2}} \longrightarrow 0
$$

is obtained. Thus there exists an isomorphism $E_{1}(-Y) \stackrel{\sim}{\rightarrow} F_{1}(-Y) \otimes L$ such that the diagram

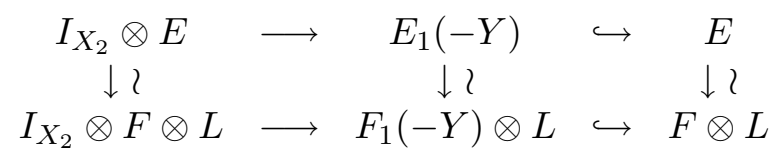


commutes. Then for the induced isomorphism $E_{1} \stackrel{\sim}{\rightarrow} F_{1} \otimes L$, the diagram

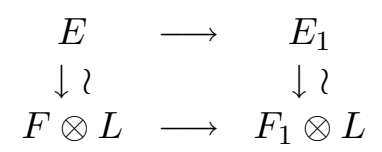

commutes. Similarly we obtain an isomorphism $E_{2} \stackrel{\sim}{\rightarrow} F_{2} \otimes L$ such that the diagram

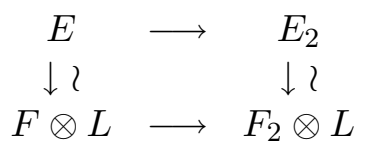

commutes. Thus we obtain an isomorphism $E_{2} / E_{2}^{\prime} \stackrel{\sim}{\rightarrow} F_{2} / F_{2}^{\prime} \otimes L$ and the exact commutative diagram:

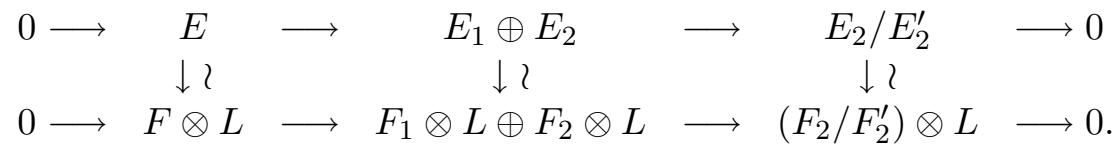

This implies that $\left(\left(E_{1}\right)_{*},\left(E_{2}\right)_{*}, \sigma\right) \sim\left(\left(F_{1}\right)_{*},\left(F_{2}\right)_{*}, \tau\right)$ and so $\Psi$ is a monomorphism.

Take $\left(\left(E_{1}\right)_{*},\left(E_{2}\right)_{*}, \sigma\right) \in \operatorname{par}-\mathcal{M}_{X}^{\left(H_{1}\right)_{*},\left(H_{2}\right)_{*}}(T)$ and put $E:=\operatorname{ker} \varphi_{\sigma}$. By definition, the canonical homomorphism $E_{1}(-Y)(t) \rightarrow E(t)$ is injective for all $t \in T$. So the exact sequence

$$
0 \longrightarrow E_{1}(-Y) \longrightarrow E \longrightarrow E \otimes \mathcal{O}_{X_{2}} \longrightarrow 0
$$

concludes that $E \otimes \mathcal{O}_{X_{2}}$ is flat over $T$ ([4], IV Proposition (11.3.7)). Similarly $E \otimes \mathcal{O}_{X_{1}}$ is also flat over $T$. Hence the proof of Proposition 1.8 implies that $E \in \mathcal{M}_{X, H_{1}^{\prime}, \tilde{H}_{2}^{\prime}}^{(1), H_{1}, \tilde{H}_{2}}(T) \cap \mathcal{M}_{X, \tilde{H}_{1}^{\prime}, H_{2}^{\prime}}^{(2), \tilde{H}_{1}, H_{2}}(T)$.

Conversely take $E \in \mathcal{M}_{X, H_{1}^{\prime}, \tilde{H}_{2}^{\prime}}^{(1), H_{1}, \tilde{H}_{2}}(T) \cap \mathcal{M}_{X, \tilde{H}_{1}^{\prime}, H_{2}^{\prime}}^{(2), \tilde{H}_{1}, H_{2}}(T)$. Let $\left(E_{1}, \tilde{E}_{2}, f_{1}\right)$ and $\left(\tilde{E}_{1}, E_{2}, f_{2}\right)$ be the corresponding triples. Then we have the following two exact sequences:

$$
\begin{aligned}
& \left.0 \longrightarrow E \longrightarrow E_{1} \oplus \tilde{E}_{2} \longrightarrow \tilde{E}_{2}\right|_{Y \times T} \longrightarrow 0 \\
& \left.0 \longrightarrow E \longrightarrow \tilde{E}_{1} \oplus E_{2} \longrightarrow \tilde{E}_{1}\right|_{Y \times T} \longrightarrow 0
\end{aligned}
$$

Consider the composition $\tilde{E}_{1}(-Y) \hookrightarrow E \rightarrow E_{1}$. Since $\tilde{E}_{1}(-Y)(t) \rightarrow E_{1}(t)$ is injective for all $t \in T, \tilde{E}_{1}(-Y) \rightarrow E_{1}$ is injective and $E_{1} / \tilde{E}_{1}(-Y)$ is flat over $T$ ([4], IV Proposition (11.3.7)). Moreover we have a factorization 
$E_{1}(-Y) \hookrightarrow \tilde{E}_{1}(-Y) \hookrightarrow E_{1}$. Similarly we have a factorization $E_{2}(-Y) \hookrightarrow$ $\tilde{E}_{2}(-Y) \hookrightarrow E_{2}$. Since $E(t) \rightarrow E_{1}(t) \oplus E_{2}(t)$ is injective for any $t \in T, E \rightarrow$ $E_{1} \oplus E_{2}$ is injective and the cokernel $E_{0}$ is flat over $T$ ([4], IV Proposition (11.3.7)). The exact sequence

$$
0 \longrightarrow \tilde{E}_{1}(-Y) \longrightarrow E \longrightarrow E_{2} \longrightarrow 0
$$

implies that $E_{1} / \tilde{E}_{1}(-Y) \cong E_{0}$. Similarly $E_{2} / \tilde{E}_{2}(-Y) \cong E_{0}$. Hence $E$ is contained in par- $\mathcal{M}_{X}^{\left(H_{1}\right)_{*},\left(H_{2}\right)_{*}}(T)$.

THEOREM 3.6. A coarse moduli scheme par- $M_{X}^{\left(H_{1}\right)_{*},\left(H_{2}\right)_{*}}$ of par$\mathcal{M}_{X}^{\left(H_{1}\right)_{*},\left(H_{2}\right)_{*}}$ exists. Moreover par- $M_{X}^{\left(H_{1}\right)_{*},\left(H_{2}\right)_{*}}$ is a subscheme of $M_{X}^{H}$ and is the scheme theoretic intersection $M_{X, H_{1}^{\prime}, \tilde{H}_{2}^{\prime}}^{(1), H_{1}, \tilde{H}_{2}} \cap M_{X, \tilde{H}_{1}^{\prime}, H_{2}^{\prime}}^{(2), \tilde{H}_{1}, H_{2}}$.

Proof. The proof is similar to Theorem 1.10.

We will give another construction of the moduli space of parabolic triples. There exists an integer $m_{0}$ such that for any integer $m \geq m_{0}$ and for any $\left(\left(E_{1}\right)_{*},\left(E_{2}\right)_{*}, \sigma\right) \in \operatorname{par}-M_{X}^{\left(H_{1}\right)_{*},\left(H_{2}\right)_{*}}(k)$,

(i) $E_{i}(m),\left(E_{i} / E_{i}^{\prime}\right)(m)$ are globally generated for $i=1,2$,

(ii) $H^{j}\left(E_{i}(m)\right)=0, H^{j}\left(\left(E_{i} / E_{i}^{\prime}\right)(m)\right)=0$ for $i=1,2$ and for any $j>0$.

For $i=1,2$, put $V_{i}:=k^{\oplus H_{i}\left(m_{0}\right)}$ and $Q_{i}:=\left\{\begin{array}{l|l}{\left[V_{i} \otimes \mathcal{O}_{X_{i}} \rightarrow E_{i}\right] \in \operatorname{Quot}_{V_{i} \otimes \mathcal{O}_{X_{i}} / X_{i} / k}^{H_{i}\left[m_{0}\right]}} & \begin{array}{l}E_{i} \text { is of pure dimension } d, \\ V_{i} \rightarrow H^{0}\left(E_{i}\right) \text { is bijective } \\ \text { and } H^{j}\left(E_{i}\right)=0 \text { for } j>0\end{array}\end{array}\right\}$.

Let $V_{i} \otimes \mathcal{O}_{X_{i} \times Q_{i}} \rightarrow \mathcal{E}_{i}$ be the universal quotient sheaf. Let $R_{i}^{\prime}$ be the Quotscheme $\operatorname{Quot}_{\mathcal{E}_{i} / X_{i} \times Q_{i} / Q_{i}}^{H_{i}\left[m_{0}\right]-\tilde{H}_{i}^{\prime}\left[m_{0}\right]}$ and $\left(\mathcal{E}_{i}\right)_{R_{i}^{\prime}} \rightarrow \mathcal{G}_{i}$ be the universal quotient sheaf. From [[19], Corollary 2.3] there exists a subscheme $R_{i}$ of $R_{i}^{\prime}$ such that for any $T \in(\mathrm{Sch} / k)$,

$$
R_{i}(T):=\left\{\begin{array}{l|l}
T \rightarrow R_{i}^{\prime} & \begin{array}{l}
\mathcal{E}_{i}(-Y)_{T} \hookrightarrow\left(\mathcal{E}_{i}\right)_{T} \rightarrow\left(\mathcal{G}_{i}\right)_{T} \text { is zero and } \\
H^{j}\left(\mathcal{G}_{i}(t)\right)=0 \text { for } j>0 \text { and for any } t \in T
\end{array}
\end{array}\right\} .
$$

Then $\left(\mathcal{G}_{i}\right)_{R_{i}}$ is an $\mathcal{O}_{Y \times R_{i}}$-module. Put $\tilde{\mathcal{E}}_{i}:=\operatorname{ker} g \otimes \mathcal{O}_{X_{i}}(Y)$, where $g$ : $\left(\mathcal{E}_{i}\right)_{R_{i}} \rightarrow\left(\mathcal{G}_{i}\right)_{R_{i}}$ is the canonical surjection. There exists a coherent sheaf $\mathcal{H}$ on $R_{1} \times R_{2}$ such that

$$
\operatorname{Hom}_{T}\left(\mathcal{H}_{T}, \mathcal{M}\right) \cong \operatorname{Hom}_{X_{T}}\left(\left(\mathcal{G}_{1}\right)_{T}, \mathcal{G}_{2} \otimes \mathcal{M}\right)
$$


for any $T \rightarrow R_{1} \times R_{2}$ and any quasi-coherent sheaf $\mathcal{M}$ on $T$ ([1], (1.1)). Let $\sigma:\left(\mathcal{G}_{1}\right)_{\mathbf{V}(\mathcal{H})} \rightarrow\left(\mathcal{G}_{2}\right)_{\mathbf{V}(\mathcal{H})}$ be the homomorphism corresponding to the canonical homomorphism $\mathcal{H} \otimes \mathcal{O}_{\mathbf{V}(\mathcal{H})} \rightarrow \mathcal{O}_{\mathbf{V}(\mathcal{H})}$. Put

$$
\varphi_{\sigma}:\left(\mathcal{E}_{1}\right)_{\mathbf{V}(\mathcal{H})} \oplus\left(\mathcal{E}_{2}\right)_{\mathbf{V}(\mathcal{H})} \longrightarrow\left(\mathcal{G}_{2}\right)_{\mathbf{V}(\mathcal{H})} ;(a, b) \mapsto \sigma(\bar{a})-\bar{b}
$$

Set

$$
P:=\left\{\begin{array}{l|l}
s \in \mathbf{V}(\mathcal{H}) & \begin{array}{l}
\sigma(s) \text { is isomorphic, } \operatorname{ker} \varphi_{\sigma}(s) \otimes \mathcal{O}_{X}\left(-m_{0}\right) \text { is stable, } \\
\chi\left(\tilde{\mathcal{E}}_{i}(s)(n)\right)=\tilde{H}_{i}\left(n+m_{0}\right) \text { and } \\
\chi\left(\mathcal{E}_{i}(-Y)(s)(n)\right)=H_{i}^{\prime}\left(n+m_{0}\right) \text { for } i=1,2
\end{array}
\end{array}\right\} .
$$

Then $\left.\operatorname{ker} \varphi_{\sigma} \otimes \mathcal{O}_{X}\left(-m_{0}\right)\right|_{X \times P}$ induces a morphism

$$
\pi: P \longrightarrow \operatorname{par}-M_{X}^{\left(H_{1}\right)_{*},\left(H_{2}\right)_{*}} .
$$

Put $G:=\left(G L\left(V_{1}\right) \times G L\left(V_{2}\right)\right) / \mathbf{G}_{m}$.

Theorem 3.7. $\pi: P \rightarrow \operatorname{par}-M_{X}^{\left(H_{1}\right)_{*},\left(H_{2}\right)_{*}}$ is a principal G-bundle.

Proof. The same arguments as proof of Theorem 2.1 shows that $\pi$ is a smooth morphism. Moreover the surjectivity of $\pi$ is obvious.

Let us consider the morphism

$$
\psi: G \times P \longrightarrow P \times{ }_{\text {par- } M_{X}^{\left(H_{1}\right)_{*},\left(H_{2}\right)_{*}} P .} P .
$$

We have only to prove that $\psi$ is an isomorphism. Note that there is a canonical isomorphism $P \times_{\operatorname{par}-\mathcal{M}_{X}^{\left(H_{1}\right)_{*},\left(H_{2}\right)_{*}}} P \cong P \times_{\operatorname{par}-M_{X}^{\left(H_{1}\right)_{*},\left(H_{2}\right)_{*}}} P$. In order to prove that $\psi$ is a monomorphism, it is sufficient to show that $\psi(R)$ is injective for all artinian local rings $R$ over $k$. Take $p \in P(R)$ and $\left[\left(g_{1}, g_{2}\right)\right] \in G(R)$ such that $\left[\left(g_{1}, g_{2}\right)\right] \cdot p=p$. Let

$$
\begin{gathered}
V_{1} \otimes \mathcal{O}_{X_{1} \times{ }_{k} R} \longrightarrow E_{1} \longrightarrow G_{1} \\
V_{2} \otimes \mathcal{O}_{X_{2} \times{ }_{k} R} \longrightarrow E_{2} \longrightarrow G_{2}
\end{gathered}
$$

be the diagram corresponding to $p$. Since $\left(g_{1}, g_{2}\right) \cdot p=p$, there are isomorphisms $\theta_{i}: E_{i} \stackrel{\sim}{\rightarrow} E_{i}, \bar{\theta}_{i}: G_{i} \stackrel{\sim}{\rightarrow} G_{i}$ for $i=1,2$ such that the diagrams

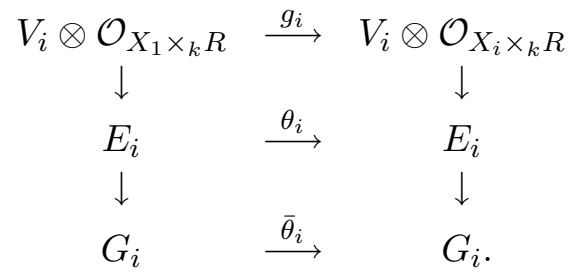


commute for $i=1,2$ and the diagram

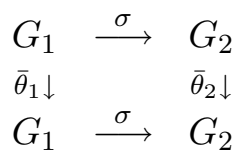

commutes. $\theta_{1}$ and $\theta_{2}$ induce an automorphism of $\operatorname{ker} \varphi_{\sigma}$. However, this automorphism is a scalar multiplication by $c \in R$, since $\operatorname{End}\left(\operatorname{ker} \varphi_{\sigma}\right)=R$. Hence $\theta_{1}=c$ and $\theta_{2}=c$, and so $\left[\left(g_{1}, g_{2}\right)\right]=1$ in $G(R)$, which proves that $\varphi(R)$ is injective.

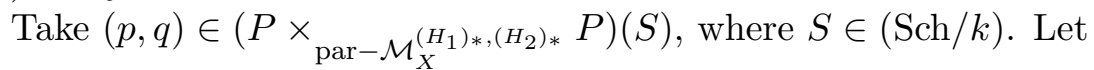

$$
\begin{aligned}
& V_{1} \otimes \mathcal{O}_{X_{1} \times_{k} S} \longrightarrow E_{1} \longrightarrow G_{1} \\
& \imath \downarrow \sigma \\
& V_{2} \otimes \mathcal{O}_{X_{2} \times{ }_{k} S} \longrightarrow E_{2} \longrightarrow G_{2}
\end{aligned}
$$

be the diagram corresponding to $p$ and

$$
\begin{gathered}
V_{1} \otimes \mathcal{O}_{X_{1} \times{ }_{k} S} \longrightarrow \bar{E}_{1} \longrightarrow \bar{G}_{1} \\
V_{2} \otimes \mathcal{O}_{X_{2} \times{ }_{k} S} \longrightarrow \bar{E}_{2} \longrightarrow \bar{G}_{2}
\end{gathered}
$$

be the diagram corresponding to $q$. From the choice of $p, q$, there exist a line bundle $\mathcal{L}$ on $S$ and an isomorphism $\theta: \operatorname{ker} \varphi_{\sigma} \stackrel{\sim}{\rightarrow} \operatorname{ker} \varphi_{\tau} \otimes \mathcal{L}$. $\theta$ induces isomorphisms $h_{1}: E_{1} \stackrel{\sim}{\rightarrow} \bar{E}_{1} \otimes \mathcal{L}, \bar{h}_{1}: G_{1} \stackrel{\sim}{\rightarrow} \bar{G}_{1} \otimes \mathcal{L}, h_{2}: E_{2} \stackrel{\sim}{\rightarrow} \bar{E}_{2} \otimes \mathcal{L}$ and $\bar{h}_{2}: G_{2} \stackrel{\sim}{\rightarrow} \bar{G}_{2} \otimes \mathcal{L}$ such that the diagram

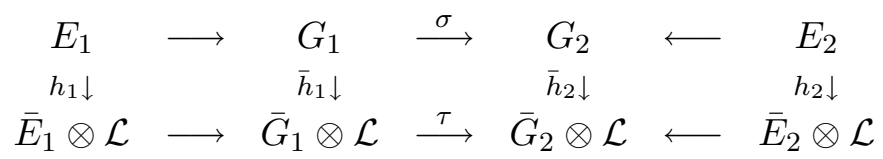

commutes. The isomorphisms $h_{1}, h_{2}$ induce isomorphisms $g_{1}: V_{1} \otimes \mathcal{O}_{S} \stackrel{\sim}{\rightarrow}$ $V_{1} \otimes \mathcal{O}_{S} \otimes \mathcal{L}$ and $g_{2}: V_{2} \otimes \mathcal{O}_{S} \stackrel{\sim}{\rightarrow} V_{2} \otimes \mathcal{O}_{S} \otimes \mathcal{L}$ such that the diagrams

$$
\begin{array}{ccc}
V_{1} \otimes \mathcal{O}_{X_{1} \times S} & \longrightarrow & E_{1} \\
g_{1} \otimes 1 \downarrow & & h_{1} \downarrow \\
V_{1} \otimes \mathcal{O}_{X_{1} \times S} \otimes \mathcal{L} & \longrightarrow & \bar{E}_{1} \otimes \mathcal{L}
\end{array}
$$

and

$$
\begin{array}{ccc}
V_{2} \otimes \mathcal{O}_{X_{2} \times S} & \longrightarrow & E_{2} \\
g_{2} \downarrow & & h_{2} \downarrow \\
V_{2} \otimes \mathcal{O}_{X_{2} \times S} \otimes \mathcal{L} & \longrightarrow & \bar{E}_{2} \otimes \mathcal{L}
\end{array}
$$

both commute. Then $\left[\left(g_{1}, g_{2}\right)\right] \cdot q=p$, which proves that $\psi(S)$ is surjective. Hence $\psi$ is an isomorphism. 


\section{§4. Local deformations of sheaves on reducible surfaces}

Lemma 4.1. Let $(A, m)$ be an artinian local ring with residue field $k=$ $A / m$ and $I$ an ideal of $A$ such that $I m=0$. Let $X$ be a projective scheme flat over $A$ and $E$ a coherent sheaf on $X \times_{A} A / I$ flat over $A / I$. Assume that there exist finite points $p_{1}, \ldots, p_{n}$ of $X \times_{A} k$ such that $E \otimes_{A} k$ is locally free over $X \times_{A} k \backslash\left\{p_{1}, \ldots, p_{n}\right\}$. Moreover assume that each stalk $E_{p_{i}}$ can be lifted to an $\mathcal{O}_{X, p_{i}}$-module $\tilde{M}_{i}$ flat over $A$. Then there exists an element $\omega(E) \in H^{2}(X \times k, \mathcal{E} n d(E \otimes k)) \otimes I$ whose vanishing is equivalent to the liftability of $E$ to an A-flat coherent sheaf $\tilde{E}$ on $X$ such that $\tilde{E}_{p_{i}} \cong M_{i}$ for each $i$.

Proof. There exists a finite covering $\left\{U_{i}\right\}_{i=1}^{r}$ of $X$ by affine open sets such that $\left.E\right|_{\left(U_{i} \cap U_{j}\right) \otimes A / I}$ is locally free for each $i, j$ with $U_{i} \neq U_{j}$ and each $\left.E\right|_{U_{i}}$ can be lifted to a coherent $\mathcal{O}_{U_{i}}$-module $\tilde{E}_{i}$ which is flat over $U_{i}$. Let $\eta_{i}$ be the element of $\operatorname{Ext}_{U_{i}}^{1}\left(\left.E\right|_{U_{i}},\left.I \otimes_{A} E\right|_{U_{i}}\right)$ corresponding to the extension

$$
\left.\left.0 \longrightarrow I \otimes_{A} E\right|_{U_{i}} \longrightarrow \tilde{E}_{i} \longrightarrow E\right|_{U_{i}} \longrightarrow 0 \text {. }
$$

Since $\left.\tilde{E}_{i}\right|_{U_{i} \cap U_{j}}$ and $\left.\tilde{E}_{j}\right|_{U_{i} \cap U_{j}}$ are locally free sheaves for $U_{i} \neq U_{j}$, there exists an isomorphism of extensions:

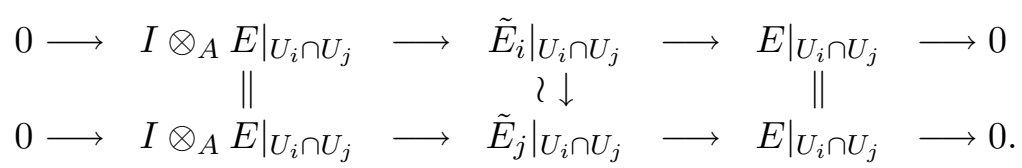

Hence we have $\left.\eta_{i}\right|_{U_{i} \cap U_{j}}=\left.\eta_{j}\right|_{U_{i} \cap U_{j}}$ and so $\left\{\eta_{i}\right\}_{i=1}^{r}$ determines an element $\eta$ of $H^{0}\left(X, \mathcal{E} x t_{X}^{1}\left(I \otimes_{A} E, E\right)\right)$. From the spectral sequence $E_{2}^{p, q}=$ $H^{p}\left(X, \mathcal{E} x t_{X}^{q}\left(I \otimes_{A} E, E\right)\right) \Rightarrow E^{p+q}=\operatorname{Ext}_{X}^{p+q}\left(I \otimes_{A} E, E\right)$, the following exact sequence is obtained:

$$
\begin{aligned}
& 0 \longrightarrow H^{1}\left(X, \mathcal{H o m}_{X}\left(I \otimes_{A} E, E\right)\right) \longrightarrow \operatorname{Ext}_{X}^{1}\left(I \otimes_{A} E, E\right) \\
& \longrightarrow H^{0}\left(X, \mathcal{E} x t_{X}^{1}\left(I \otimes_{A} E, E\right)\right) \longrightarrow H^{2}\left(X, \mathcal{H o m}_{X}\left(I \otimes_{A} E, E\right)\right) .
\end{aligned}
$$

Let $\omega$ be the image of $\eta$ by $H^{0}\left(X, \mathcal{E} x t_{X}^{1}\left(I \otimes_{A} E, E\right)\right) \rightarrow H^{2}\left(X, \mathcal{H o m}_{X}\left(I \otimes_{A}\right.\right.$ $E, E))=H^{2}\left(X \times_{A} k, \mathcal{E} n d_{X}\left(E \otimes_{A} k\right) \otimes_{k} I\right)$. Then $\omega=0$ if and only if $\eta$ comes from an element of $\operatorname{Ext}_{X}^{1}\left(I \otimes_{A} E, E\right)$, that is an extension $0 \rightarrow$ $I \otimes_{A} E \rightarrow \tilde{E} \rightarrow E \rightarrow 0$ such that

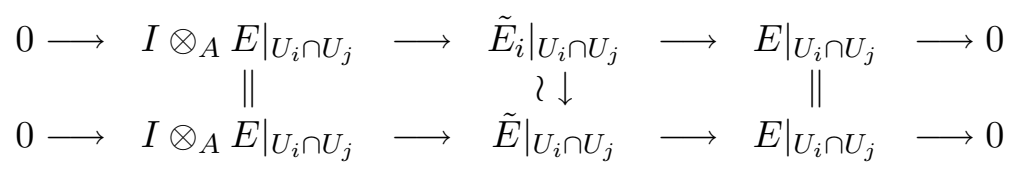


for each $i$. Hence $\omega$ is the desired obstruction.

Let $R$ be a discrete valuation ring and $k=R / m_{R}$ be the residue field. (We assume that $k=\bar{k}$.) Let $t_{0}$ be the closed point of $\operatorname{Spec} R$ and $\eta$ be the generic point of $\operatorname{Spec} R$. Let $\tilde{X}$ be a projective scheme flat over $R$ such that $\tilde{X}_{\eta}$ is a smooth surface and $X:=\tilde{X}_{t_{0}}=X_{1} \cup X_{2}$, where $X_{1}$ and $X_{2}$ are smooth surfaces and $Y:=X_{1} \cap X_{2}$ is a smooth curve. We will investigate which sheaves on $X$ can be lifted to sheaves on $\tilde{X}$.

Proposition 4.2. Let $E$ be a coherent sheaf of pure dimension 2 on $X$ such that rank $\left.E\right|_{X_{1}} \neq\left.\operatorname{rank} E\right|_{X_{2}}$. Then $E$ can not be lifted to a coherent sheaf on $\tilde{X}$ flat over $R$.

Proof. Take a very ample line bundle $\mathcal{O}_{\tilde{X}}(1)$. Since $\tilde{X}$ is flat over $R$, we may assume that $H^{0}\left(\tilde{X}, \mathcal{O}_{\tilde{X}}(1)\right)$ is a projective module over $R$ and that $H^{0}\left(\tilde{X}, \mathcal{O}_{\tilde{X}}(1)\right) \otimes k\left(t_{0}\right) \cong H^{0}\left(X, \mathcal{O}_{X}(1)\right)$, where $\mathcal{O}_{X}(1):=\left.\mathcal{O}_{\tilde{X}}(1)\right|_{X}$. Put $r_{i}:=\left.\operatorname{rank} E\right|_{X_{i}}$ for $i=1,2$. Assume that $E$ is lifted to a coherent sheaf $\tilde{E}$ on $\tilde{X}$ flat over $R$. Take general sections $s_{1}, s_{2} \in H^{0}\left(\tilde{X}, \mathcal{O}_{\tilde{X}}(1)\right)$. Let $Z\left(s_{i}\right) \subset \tilde{X}$ be the zero scheme of $s_{i}$. Then we may assume that $\left.\tilde{E}\right|_{Z\left(s_{1}\right) \cap Z\left(s_{2}\right)}$ is flat over $R,\left.\tilde{E}\right|_{Z\left(s_{1}\right) \cap Z\left(s_{2}\right) \cap \tilde{X}_{\eta}}$ is a locally free sheaf of rank $r:=\left.\operatorname{rank} \tilde{E}\right|_{\tilde{X}_{\eta}}$ and $\left.E\right|_{Z\left(s_{1}\right) \cap Z\left(s_{2}\right) \cap X_{i}}$ are locally free sheaves of rank $r_{i}$ on $Z\left(s_{1}\right) \cap Z\left(s_{2}\right) \cap$ $X_{i}$ for $i=1,2$. Then $\left.\tilde{E}\right|_{Z\left(s_{1}\right) \cap Z\left(s_{2}\right)}$ is a locally free sheaf on $Z\left(s_{1}\right) \cap$ $Z\left(s_{2}\right)$ and its rank is $r_{1}=\left.\operatorname{rank} \tilde{E}\right|_{Z\left(s_{1}\right) \cap Z\left(s_{2}\right) \cap X_{1}}=\left.\operatorname{rank} \tilde{E}\right|_{Z\left(s_{1}\right) \cap Z\left(s_{2}\right) \cap \tilde{X}_{\eta}}=$ $\left.\operatorname{rank} \tilde{E}\right|_{Z\left(s_{1}\right) \cap Z\left(s_{2}\right) \cap X_{2}}=r_{2}$, which is a contradiction.

Let $E$ be a coherent sheaf of pure dimension 2 on $X$ and $\left(E^{(1)}, \tilde{E}^{(2)}, f\right)$ be the corresponding triple. Assume that $E^{(1)}$ and $\tilde{E}^{(2)}$ are of rank $r$, locally free along $Y$ and $f:\left.\left.E^{(1)}\right|_{Y} \rightarrow \tilde{E}^{(2)}\right|_{Y}$ is injective. Let $\left\{p_{1}, \ldots, p_{n}\right\}$ be the set of points of $Y$ where $f$ is not an isomorphism. Assume that coker $f_{p_{i}} \cong k\left(p_{i}\right)$ for each $i$. Let $M_{i}$ be the stalk of $E$ at $p_{i}$.

Let $\mathcal{C}_{R}$ be the category of artinian local rings $A$ over $R$ with $A / m_{A}=k$.

We define a functor

$$
D_{E}: \mathcal{C}_{R} \longrightarrow(\text { Sets })
$$

by

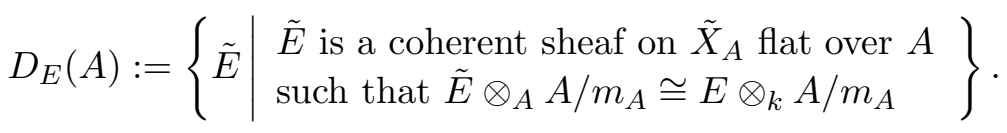


We also define a functor

$$
D_{M_{i}}: \mathcal{C}_{R} \longrightarrow(\text { Sets })
$$

by

$$
D_{M_{i}}(A):=\left\{\begin{array}{l|l}
\tilde{M} & \begin{array}{l}
\tilde{M} \text { is an } \mathcal{O}_{\tilde{X}_{A}} \text {-module flat over } A \\
\text { such that } \tilde{M} \otimes_{A} A / m_{A} \cong M_{i} \otimes_{k} A / m_{A}
\end{array}
\end{array}\right\} .
$$

Then we can define a morphism of functors

$$
\Phi_{1}: D_{E} \longrightarrow D_{M_{1}} \times \cdots \times D_{M_{n}}
$$

by $\Phi_{1}(\tilde{E}):=\left(\tilde{E}_{p_{1}}, \ldots, \tilde{E}_{p_{n}}\right)$. If $H^{2}(X, \mathcal{E} n d(E, E))=0$, then from Lemma 4.1, $\Phi_{1}$ is formally smooth. So we will study $D_{M_{i}}$. Put $M:=M_{i}$ and $p:=p_{i}$. There is an exact sequence

$$
0 \longrightarrow M \longrightarrow \mathcal{O}_{X_{1}, p}^{\oplus r} \oplus \mathcal{O}_{X_{2}, p}^{\oplus r} \stackrel{\varphi_{f_{p}}}{\longrightarrow} \mathcal{O}_{Y, p}^{\oplus r} \longrightarrow 0
$$

Let $\tilde{f}: \mathcal{O}_{X_{1}, p}^{\oplus r} \rightarrow \mathcal{O}_{X_{1}, p}^{\oplus r}$ be a lift of $f_{p}: \mathcal{O}_{Y, p}^{\oplus r} \rightarrow \mathcal{O}_{Y, p}^{\oplus r}$. Then the following exact commutative diagram is obtained:

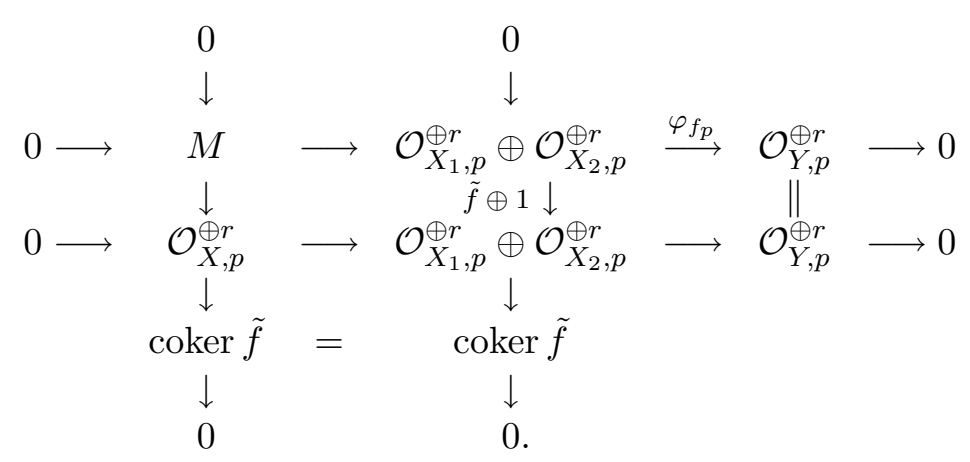

We consider $M$ as a submodule of $\mathcal{O}_{X, p}^{\oplus r}$ with respect to this diagram. We define a functor

$$
D_{M \subset \mathcal{O}_{X, p}^{\oplus r}}: \mathcal{C}_{R} \longrightarrow(\text { Sets })
$$

by

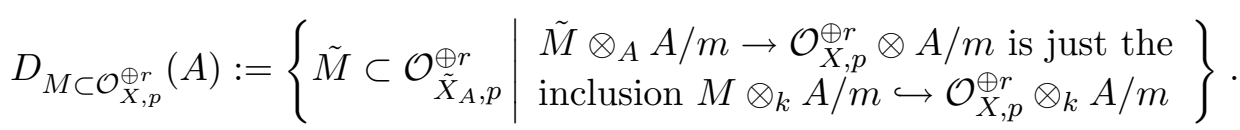

Let $I_{X_{i}}$ be the ideal of $\mathcal{O}_{X}$ corresponding to the subscheme $X_{i} \subset X$ for $i=1,2$. Then $I_{X_{1}} \cong \mathcal{O}_{X_{2}}(-Y)$ and so $I_{X_{1}, p}$ is a principal ideal. So we can write $I_{X_{1}, p}=\left(f_{1}\right)$ and $I_{X_{2}, p}=\left(f_{2}\right)$. 
LEMMA 4.3. $\operatorname{Ext}_{\mathcal{O}_{X, p}}^{i}\left(M, \mathcal{O}_{X, p}\right)=0$ for $i \geq 1$.

Proof. We have the following free resolution of $\mathcal{O}_{X_{1}, p}$ :

$$
\cdots \longrightarrow \mathcal{O}_{X, p} \stackrel{f_{1}}{\longrightarrow} \mathcal{O}_{X, p} \stackrel{f_{2}}{\longrightarrow} \mathcal{O}_{X, p} \stackrel{f_{1}}{\longrightarrow} \mathcal{O}_{X, p} \longrightarrow \mathcal{O}_{X_{1}, p} \longrightarrow 0 .
$$

Taking the dual of this complex, we have the following exact sequence:

$$
\mathcal{O}_{X, p}^{\vee} \stackrel{f_{1}}{\longrightarrow} \mathcal{O}_{X, p}^{\vee} \stackrel{f_{2}}{\longrightarrow} \mathcal{O}_{X, p}^{\vee} \stackrel{f_{1}}{\longrightarrow} \cdots
$$

Thus $\operatorname{Ext}_{\mathcal{O}_{X, p}}^{i}\left(\mathcal{O}_{X_{1}, p}, \mathcal{O}_{X, p}\right)=0$ for $i \geq 1$. Similarly $\operatorname{Ext}_{\mathcal{O}_{X, p}}^{i}\left(\mathcal{O}_{X_{2}, p}, \mathcal{O}_{X, p}\right)=$ 0 for $i \geq 1$. There is an isomorphism $I_{Y, p} \cong I_{X_{1}, p} \oplus I_{X_{2}, p}$ and an exact sequence

$$
\operatorname{Ext}_{\mathcal{O}_{X, p}}^{i-1}\left(I_{Y, p}, \mathcal{O}_{X, p}\right) \longrightarrow \operatorname{Ext}_{\mathcal{O}_{X, p}}^{i}\left(\mathcal{O}_{Y, p}, \mathcal{O}_{X, p}\right) \longrightarrow \operatorname{Ext}_{\mathcal{O}_{X, p}}^{i}\left(\mathcal{O}_{X, p}, \mathcal{O}_{X, p}\right)
$$

Thus $\operatorname{Ext}_{\mathcal{O}_{X, p}}^{i}\left(\mathcal{O}_{Y, p}, \mathcal{O}_{X, p}\right)=0$ for $i \geq 2$. From the exact sequence

$$
0 \longrightarrow M \longrightarrow \mathcal{O}_{X_{1}, p}^{\oplus r} \oplus \mathcal{O}_{X_{2}, p}^{\oplus r} \stackrel{\varphi_{f_{p}}}{\longrightarrow} \mathcal{O}_{Y, p}^{\oplus r} \longrightarrow 0,
$$

the following exact sequence is obtained:

$\operatorname{Ext}_{\mathcal{O}_{X, p}}^{i}\left(\mathcal{O}_{X_{1}, p}^{\oplus r} \oplus \mathcal{O}_{X_{2}, p}^{\oplus r}, \mathcal{O}_{X, p}\right) \longrightarrow \operatorname{Ext}_{\mathcal{O}_{X, p}}^{i}\left(M, \mathcal{O}_{X, p}\right) \longrightarrow \operatorname{Ext}_{\mathcal{O}_{X, p}}^{i+1}\left(\mathcal{O}_{Y, p}^{\oplus r}, \mathcal{O}_{X, p}\right)$

Hence we have $\operatorname{Ext}_{\mathcal{O}_{X, p}}^{i}\left(M, \mathcal{O}_{X, p}\right)=0$ for $i \geq 1$.

We will use the following lemma.

Lemma 4.4. Let $A \rightarrow B$ be a local homomorphism of noetherian local rings and $k=A / m$ be the residue field. Let $M$ be a $B$-module of finite type which is flat over $A$ and satisfies $\operatorname{Ext}_{B \otimes_{A} k}^{1}\left(M \otimes_{A} k, B \otimes_{A} k\right)=0$. Then $\operatorname{Hom}_{B}(M, B)$ is flat over $A$ and $\operatorname{Hom}_{B}(M, B) \otimes_{A} k=\operatorname{Hom}_{B \otimes_{A} k}\left(M \otimes_{A}\right.$ $\left.k, B \otimes_{A} k\right)$.

Proof. See [12], Appendix.

We define a morphism of functors

$$
\Phi_{2}: D_{M \subset \mathcal{O}_{X, p}^{\oplus r}} \longrightarrow D_{M}
$$

by putting $\Phi_{2}\left(\tilde{M} \subset \mathcal{O}_{\tilde{X}_{A}, p}^{\oplus r}\right):=\tilde{M}$. 
Take any $A \in \mathcal{C}_{R}$ and an ideal $I \subset A$ such that $I m=0$. If $\tilde{M} \in D_{E}(A)$ and an injection $\tilde{M} \otimes_{A} A / I \subset \mathcal{O}_{\tilde{X}_{A / I}^{\oplus r}, p}^{\oplus r}$ is given, then from Lemma 4.3 and Lemma 4.4, $\operatorname{Hom}\left(\tilde{M}, \mathcal{O}_{\tilde{X}_{A}, p}^{\oplus r}\right) \rightarrow \operatorname{Hom}\left(\tilde{M} \otimes_{A} A / I, \mathcal{O}_{\tilde{X}_{A / I, p} r}^{\oplus r}\right)$ is surjective. So the injection $\tilde{M} \otimes_{A} A / I \subset \mathcal{O}_{\tilde{X}_{A / I}, p}^{\oplus r}$ can be lifted to an injection $\tilde{M} \subset \mathcal{O}_{\tilde{X}_{A}, p}^{\oplus r}$. Hence $\Phi_{2}$ is formally smooth.

Let $N$ be the cokernel of the injection $M \hookrightarrow \mathcal{O}_{X, p}^{\oplus r}$. We define a functor

$$
D_{N}: \mathcal{C}_{R} \longrightarrow(\text { Sets })
$$

by

$$
D_{N}(A):=\left\{\begin{array}{l|l}
\tilde{N} & \begin{array}{l}
\tilde{N} \text { is an } \mathcal{O}_{\tilde{X}_{A}, p} \text {-module flat over } A \\
\text { such that } \tilde{N} \otimes_{A} A / m \cong N \otimes_{k} A / m
\end{array}
\end{array}\right\}
$$

Then we obtain the following morphism of functors;

$$
\Phi_{3}: D_{M \subset \mathcal{O}_{X, p}^{\oplus r}}^{\oplus r} D_{N} ; \quad\left[\tilde{M} \subset \mathcal{O}_{\tilde{X}_{A}, p}^{\oplus r}\right] \mapsto \mathcal{O}_{\tilde{X}_{A}, p}^{\oplus r} / \tilde{M}
$$

It is obvious that $\Phi_{3}$ is formally smooth.

By the assumption on $f:\left.\left.E^{(1)}\right|_{Y} \rightarrow \tilde{E}^{(2)}\right|_{Y}, N$ is generated by one element. So there is an exact sequence

$$
0 \longrightarrow I_{N} \longrightarrow \mathcal{O}_{X, p} \longrightarrow N \longrightarrow 0 \text {. }
$$

We define a functor $D_{I_{N} \subset \mathcal{O}_{X, p}}$ in the same way as $D_{M \subset \mathcal{O}_{X, p}^{\oplus r}}$ and a morphism of functors $\Phi_{4}: D_{I_{N} \subset \mathcal{O}_{X, p}} \rightarrow D_{N}$ in the same way as $\Phi_{3}$.

Now let $\tilde{f}_{1} \in \mathcal{O}_{\tilde{X}, p}$ and $\tilde{f}_{2} \in \mathcal{O}_{\tilde{X}, p}$ be lifts of $f_{1}$ and $f_{2}$ respectively. Then there exists an element $g \in \mathcal{O}_{\tilde{X}, p}$ such that $\tilde{f}_{1} \tilde{f}_{2}=t g$ where $t \in R$ is a local parameter.

Proposition 4.5. Let $A \in \mathcal{C}_{R}$ and $\tilde{M}$ be an element of $D_{M}(A)$. If $g$ is a unit in $\mathcal{O}_{\tilde{X}, p}$, then $t A=0$.

Proof. Since $\Phi_{2}, \Phi_{4}$ are formally smooth, $\tilde{M}$ induces an $A$-valued point $\left[\begin{array}{lll}\tilde{I} & \subset \mathcal{O}_{\tilde{X}_{A}, p}\end{array}\right]$ of $D_{I_{N} \subset \mathcal{O}_{X, p}} . \quad I_{N}$ is generated by $f_{1}$ and another element $h \in \mathcal{O}_{X, p}$. Then the induced homomorphism $\mathcal{O}_{Y, p} \stackrel{\bar{h}}{\rightarrow} \mathcal{O}_{Y, p}$ is injective. 
From the exact commutative diagram

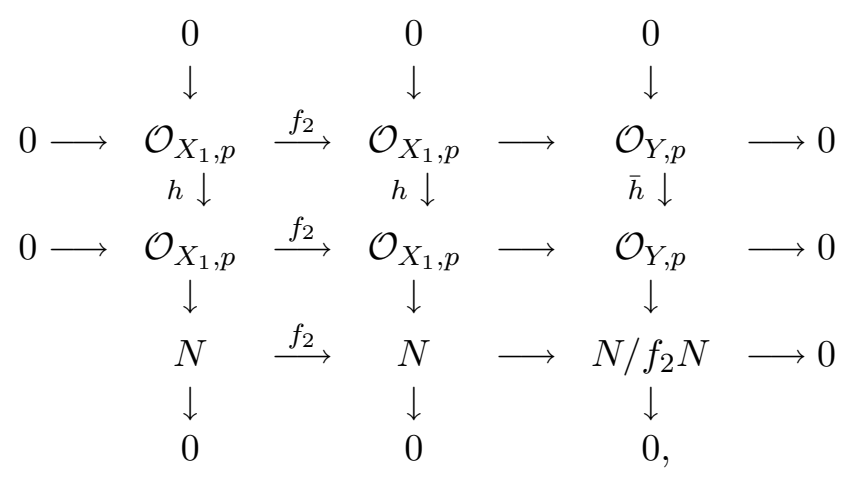

one sees that $N \stackrel{f_{2}}{\rightarrow} N$ is injective. $\tilde{N}:=\mathcal{O}_{\tilde{X}_{A}, p} / \tilde{I}$ is flat over $A$ by definition. Thus $\tilde{N} \stackrel{f_{2}}{\longrightarrow} \tilde{N}$ is injective and $\tilde{N} / \tilde{f}_{2} \tilde{N}$ is flat over $A$ ([4] IV Proposition (11.3.7)). On the other hand, since $g \in \mathcal{O}_{\tilde{X}}^{\times}, t=-g^{-1} \tilde{f}_{1} \tilde{f}_{2}$. Thus $t\left(\tilde{N} / \tilde{f}_{2} \tilde{N}\right)=0$. Since $\tilde{N} / \tilde{f}_{2} \tilde{N}$ is faithfully flat over $A$, we have $t A=0$.

From Proposition 4.5, we can see that $M$ can never be lifted to a sheaf on $\tilde{X}$ if $g$ is a unit in $\mathcal{O}_{\tilde{X}, p}$.

The following proposition is useful for the lifting problem of sheaves on a degenerate quadric surface in the next section (Theorem 5.4).

Proposition 4.6. Assume that the image $\bar{g} \in \mathcal{O}_{Y, p}$ of $g$ is a regular parameter. Then $M$ can be lifted to an $\mathcal{O}_{\tilde{X}_{R}, p}$-module flat over $R$.

Proof. There is an exact sequence

$$
0 \longrightarrow \mathcal{O}_{X_{1}, p} \stackrel{h}{\longrightarrow} \mathcal{O}_{X_{1}, p} \longrightarrow N \longrightarrow 0
$$

for some $h \in \mathcal{O}_{X_{1}, p} . h$ can be written as $h=u g+f_{2} \varphi$ where $u \in \mathcal{O}_{X_{1}, p}^{\times}$and $\varphi \in \mathcal{O}_{X_{1}, p}$. Let $\tilde{u}$ and $\tilde{\varphi}$ be lifts of $u$ and $\varphi$ to $\mathcal{O}_{\tilde{X}, p}$ respectively. Let $\tilde{I}$ be the ideal of $\mathcal{O}_{\tilde{X}, p}$ generated by $\tilde{u} g+\tilde{f}_{2} \tilde{\varphi}, \tilde{f}_{1}+t \tilde{u}^{-1} \tilde{\varphi}$. Since there is an equality $\left(\tilde{f}_{1}+t \tilde{u}^{-1} \tilde{\varphi}\right) \tilde{f}_{2}-t \tilde{u}^{-1}\left(\tilde{u} g+\tilde{f}_{2} \tilde{\varphi}\right)=0$, we can define a homomorphism

$$
\mathcal{O}_{\tilde{X}, p} /\left(\tilde{f}_{2}, \tilde{u} g+\tilde{f}_{2} \tilde{\varphi}\right) \stackrel{\tilde{f}_{1}+t \tilde{u}^{-1} \tilde{\varphi}}{\longrightarrow} \mathcal{O}_{\tilde{X}, p} /\left(\tilde{u} g+\tilde{f}_{2} \tilde{\varphi}\right) .
$$

Since $\mathcal{O}_{X, p} \stackrel{\tilde{u} g+f_{2} \tilde{\varphi}}{\longrightarrow} \mathcal{O}_{X, p}$ is injective, $\mathcal{O}_{\tilde{X}, p} \stackrel{\tilde{u} g+\tilde{f}_{2} \tilde{\varphi}}{\longrightarrow} \mathcal{O}_{\tilde{X}, p}$ is injective and $\mathcal{O}_{\tilde{X}, p} /\left(\tilde{u} g+\tilde{f}_{2} \tilde{\varphi}\right)$ is flat over $R([4]$, IV Proposition (11.3.7)). Let us consider 
the diagram

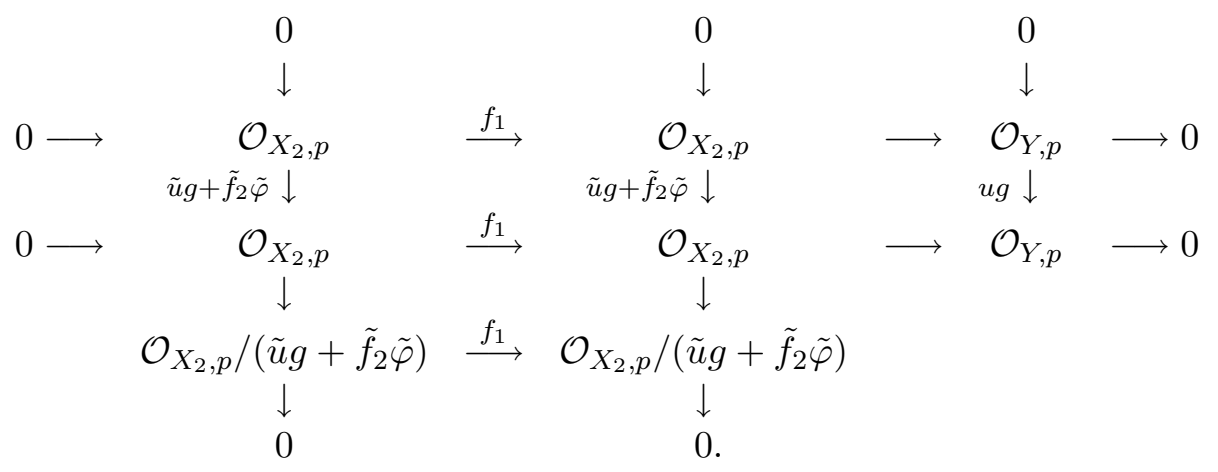

Then the injectivity of $\mathcal{O}_{Y, p} \stackrel{u g}{\rightarrow} \mathcal{O}_{Y, p}$ implies that $\mathcal{O}_{X_{2}, p} /\left(\tilde{u} g+\tilde{f}_{2} \tilde{\varphi}\right) \stackrel{f_{1}}{\rightarrow}$ $\mathcal{O}_{X_{2}, p} /\left(\tilde{u} g+\tilde{f}_{2} \tilde{\varphi}\right)$ is injective, and so $\mathcal{O}_{X_{2}, p} /\left(\tilde{u} g+\tilde{f}_{2} \tilde{\varphi}\right) \stackrel{f_{1}}{\longrightarrow} \mathcal{O}_{X, p} /(\tilde{u} g+$ $\left.\tilde{f}_{2} \tilde{\varphi}\right)$ is injective. Hence the homomorphism $\mathcal{O}_{\tilde{X}, p} /\left(\tilde{f}_{2}, \tilde{u} g+\tilde{f}_{2} \tilde{\varphi}\right) \stackrel{\tilde{f}_{1}+t \tilde{u}^{-1} \tilde{\varphi}}{\longrightarrow}$ $\mathcal{O}_{\tilde{X}, p} /\left(\tilde{u} g+\tilde{f}_{2} \tilde{\varphi}\right)$ is injective and $\mathcal{O}_{\tilde{X}, p} /\left(\tilde{f}_{1}+t \tilde{u}^{-1} \tilde{\varphi}, \tilde{u} g+\tilde{f}_{2} \tilde{\varphi}\right)=\mathcal{O}_{\tilde{X}, p} / \tilde{I}$ is flat over $R\left([4], I V\right.$ Proposition (11.3.7)). Hence $\left[\tilde{I} \subset \mathcal{O}_{\tilde{X}, p}\right]$ is an element of $D_{I_{N} \subset \mathcal{O}_{X, p}}(R)$. Since $\Phi_{3}(R)$ is surjective, there exists an element $\left[\tilde{M} \subset \mathcal{O}_{\tilde{X}, p}^{\oplus r}\right]$ of $D_{M \subset \mathcal{O}_{X, p}^{\oplus r}}(R)$ such that $\mathcal{O}_{\tilde{X}, p}^{\oplus r} / \tilde{M} \cong \mathcal{O}_{\tilde{X}, p} / \tilde{I}$. $\tilde{M}$ is the desired lift of $M$.

\section{$\S 5$. Moduli space of rank 2 stable sheaves on a reducible quadric surface}

Let $H_{1}, H_{2}$ be two distinct planes in $\mathbf{P}^{3}$ and put $Q_{0}:=H_{1} \cup H_{2}$. We consider the reduced structure on $Q_{0}$. We put $L:=H_{1} \cap H_{2}$. Then $Q_{0}$ satisfies the hypothesis $(\dagger)$ in section 1 . We consider the polarization with respect to $\mathcal{O}_{Q_{0}}(1):=\left.\mathcal{O}_{\mathbf{P}^{3}}(1)\right|_{Q_{0}}$. Fix a positive integer $n_{0}$ and put $P_{n_{0}}^{(0,0)}(m):=$ $4\left(\begin{array}{c}m+2 \\ 2\end{array}\right)-2(m+1)-n_{0}$. For an integer $n$, put $P_{n}^{(0)}(m):=2\left(\begin{array}{c}m+2 \\ 2\end{array}\right)-n$, $P_{n}^{(-1)}(m):=2\left(\begin{array}{c}m+2 \\ 2\end{array}\right)-(m+1)-n$ and $P_{n}^{(1)}(m):=2\left(\begin{array}{c}m+2 \\ 2\end{array}\right)+(m+1)+1-n$. We denote $\mathcal{M}_{Q_{0}, P_{1}(m-1), P_{2}(m-1)}^{(1), P_{1}(m), P_{2}(m)}$ simply by $\mathcal{M}_{(1), Q_{0}}^{P_{1}, P_{2}}$. We write $M_{(1), Q_{0}}^{P_{1}, P_{2}}, \mathcal{M}_{(2), Q_{0}}^{P_{1}, P_{2}}$ and $M_{(2), Q_{0}}^{P_{1}, P_{2}}$ similarly. If we put

$$
M_{Q_{0}}^{\circ} P_{n_{0}}^{(0,0)}:=\left\{E \in M_{Q_{0}}^{P_{n_{0}}^{(0,0)}}|\operatorname{rank} E|_{H_{1}}=\left.\operatorname{rank} E\right|_{H_{2}}=2\right\},
$$

then by Proposition 1.13 it is an open subscheme of $M_{Q_{0}}^{P_{n_{0}}^{(0,0)}}$. 
THEOREM 5.1. Let $n_{0}$ be an integer with $n_{0} \geq 4$. Then

$$
\begin{aligned}
& M_{Q_{0}}^{\circ}{ }_{n_{0}^{(0,0)}}^{(0)}= \\
& \bigcup_{\substack{n_{1}+n_{2}=n_{0} \\
n_{1} \geq 0, n_{2} \geq 0}} M_{(1), Q_{0}}^{P_{n_{1}}^{(0)}, P_{n_{2}}^{(0)}} \cup \bigcup_{\substack{n_{1}+n_{2}=n_{0} \\
0<n_{1}<n_{2}}}^{\bigcup} M_{(1), Q_{0}}^{P_{n_{1}}^{(-1)}, P_{n_{2}}^{(1)}} \cup \underset{\substack{n_{1}+n_{2}=n_{0} \\
n_{1}>n_{2}>0}}{\bigcup} M_{(2), Q_{0}}^{P_{n_{1}}^{(1)}, P_{n_{2}}^{(-1)}} .
\end{aligned}
$$

Moreover each component of the right hand side is non-empty.

Proof. Let $E$ be an element of $M^{\circ}{ }_{Q_{0}}^{P_{n_{0}}^{(0,0)}}(k)$. We have the following exact sequence:

$$
\left.0 \longrightarrow E \longrightarrow E^{(1)} \oplus \tilde{E}^{(2)} \longrightarrow \tilde{E}^{(2)}\right|_{L} \longrightarrow 0 .
$$

Since $\operatorname{rank} E^{(1)}=\operatorname{rank} \tilde{E}^{(2)}=2$, we have

$$
\begin{aligned}
\chi(E(m))= & \chi\left(E^{(1)}(m)\right)+\chi\left(\tilde{E}^{(2)}(m)\right)-\chi\left(\left.\tilde{E}^{(2)}\right|_{L}(m)\right) \\
= & 4\left(\begin{array}{c}
m+2 \\
2
\end{array}\right)+\left(c_{1}\left(E^{(1)}\right)+c_{1}\left(\tilde{E}^{(2)}\right)-2\right)(m+1)-c_{2}\left(E^{(1)}\right) \\
& \quad-c_{2}\left(\tilde{E}^{(2)}\right)+\frac{c_{1}\left(E^{(1)}\right)^{2}+c_{1}\left(E^{(1)}\right)+c_{1}\left(\tilde{E}^{(2)}\right)^{2}-c_{1}\left(\tilde{E}^{(2)}\right)}{2} .
\end{aligned}
$$

If we put $a:=c_{1}\left(E^{(1)}\right)$, then we have $c_{1}\left(\tilde{E}^{(2)}\right)=-a$ since $\chi(E(m))=$ $P_{n_{0}}^{(0,0)}(m)$. If we assume $a \leq-2$, then $\mu^{S}\left(E^{(1)}\right) \leq-1<-1 / 2=\mu^{S}(E)$ which contradicts the stability of $E$. (Recall that we defined $\mu^{S}(E)=$ $a_{1}(E) / a_{0}(E)$.) Assume that $a \geq 2$. For the subsheaf $E^{(1)}(-L) \subset E$, we have $\mu^{S}\left(E^{(1)}(-L)\right)=a / 2-1 \geq 0>-1 / 2=\mu^{S}(E)$ which also contradicts the stability of $E$. Hence we have $-1 \leq a \leq 1$.

Case 1. $a=-1$

In this case

$$
\begin{aligned}
\chi\left(E^{(1)}(m)\right) & =2\left(\begin{array}{c}
m+2 \\
2
\end{array}\right)-(m+1)-c_{2}\left(E^{(1)}\right) \\
\chi\left(\tilde{E}^{(2)}(m)\right) & =2\left(\begin{array}{c}
m+2 \\
2
\end{array}\right)+(m+1)+1-c_{2}\left(\tilde{E}^{(2)}\right) \text { and } \\
\chi(E(m)) & =4\left(\begin{array}{c}
m+2 \\
2
\end{array}\right)-2(m+1)-c_{2}\left(E^{(1)}\right)-c_{2}\left(\tilde{E}^{(2)}\right) .
\end{aligned}
$$


Thus $E \in M_{1}^{P_{n_{1}}^{(-1)}, P_{n_{2}}^{(1)}}(k)$, where $n_{1}=c_{2}\left(E^{(1)}\right)$ and $n_{2}=c_{2}\left(\tilde{E}^{(2)}\right)$. The equality $\chi(E(m))=P_{n_{0}}^{(0,0)}(m)$ implies that $n_{1}+n_{2}=n_{0}$. Since $E$ is stable, $\chi(E(m)) / 4<\chi\left(E^{(1)}(m)\right) / 2$ for all sufficiently large integers $m$. Hence we have the inequality $n_{1}<n_{2}$. If $E^{(1)}$ is not $\mu$-semi-stable, there exists a quotient coherent sheaf $F$ of $E^{(1)}$ of rank 1 , such that $\mu^{S}\left(E^{(1)}\right)=-1 / 2>$ $-1=\mu^{S}(F)$. Since $F$ is a quotient sheaf of $E$ and $\mu^{S}(E)=-1 / 2>\mu^{S}(F)$, this contradicts the stability of $E$. Hence $E^{(1)}$ is $\mu$-semi-stable. From Schwarzenberger's inequality, we have $n_{1}=c_{2}\left(E^{(1)}\right) \geq c_{1}\left(E^{(1)}\right)^{2} / 4>0$.

Case 2. $a=1$

Assume that the homomorphism $\left.\left.E^{(1)}\right|_{L} \rightarrow \tilde{E}^{(2)}\right|_{L}$ is not isomorphic at the generic point of $L$. If $F$ is the kernel of the homomorphism $E^{(1)} \rightarrow$ $\left.\tilde{E}^{(2)}\right|_{L}$, then $F$ is a subsheaf of $E$ and $c_{1}(F) \geq c_{1}\left(E^{(1)}\right)-1=0$ which contradicts the stability of $E$. Hence the homomorphism $\left.\left.E^{(1)}\right|_{L} \rightarrow \tilde{E}^{(2)}\right|_{L}$ is isomorphic at the generic point of $L$. So we have $c_{1}\left(\tilde{E}^{(1)}\right)=1$ and $c_{1}\left(E^{(2)}\right)=-1$. From the same argument as Case 1, $E \in M_{(2), Q_{0}}^{P_{n_{1}}^{(1)}, P_{n_{2}}^{(-1)}}(k)$ for integers $n_{1}, n_{2}$ with $n_{1}+n_{2}=n_{0}$ and $n_{1}>n_{2}>0$.

Case 3. $a=0$

If $F$ is a rank 1 quotient coherent sheaf of $E^{(1)}, \mu^{S}(F) \geq-1 / 2=$ $\mu^{S}(E)$. Hence we have $\mu^{S}(F) \geq 0=\mu^{S}\left(E^{(1)}\right)$ and so $E^{(1)}$ is $\mu$-semistable. From Schwarzenberger's inequality, we have $c_{2}\left(E^{(1)}\right) \geq 0$. Take any rank 1 quotient coherent sheaf $F$ of $\tilde{E}^{(2)}$. If we put $F^{\prime}$ the image of the homomorphism $E \rightarrow F$, we have $\mu^{S}(F) \geq \mu^{S}\left(F^{\prime}\right)>\mu^{S}(E)=-1 / 2$. Hence we have $\mu^{S}(F) \geq 0=\mu^{S}\left(\tilde{E}^{(2)}\right)$ and so $\tilde{E}^{(2)}$ is $\mu$-semi-stable. So we have $c_{2}\left(\tilde{E}^{(2)}\right) \geq 0$. Hence $E \in M_{(1), Q_{0}}^{P_{n_{1}}^{(0)}, P_{n_{2}}^{(0)}}(k)$ for integers $n_{1}, n_{2}$ with $n_{1} \geq 0, n_{2} \geq 0$ and $n_{1}+n_{2}=n_{0}$. Thus we have proved the first part of the theorem.

Take integers $n_{1}, n_{2}$ with $n_{1}+n_{2}=n_{0}$ and $0<n_{1}<n_{2}$. We will show that $M_{(1), Q_{0}}^{P_{n_{1}}^{(-1)}, P_{n_{2}}^{(1)}} \neq \emptyset$. There exist rank 2 stable bundles $E_{1}$ on $H_{1}$ and $\tilde{E}_{2}$ on $H_{2}$ such that $\chi\left(E_{1}(m)\right)=P_{n_{1}}^{(-1)}(m), \chi\left(\tilde{E}_{2}(m)\right)=P_{n_{2}}^{(1)}(m)$, $\left.E_{1}\right|_{L} \cong \mathcal{O}_{L}(-1) \oplus \mathcal{O}_{L}$ and $\left.\tilde{E}_{2}\right|_{L} \cong \mathcal{O}_{L}(1) \oplus \mathcal{O}_{L}$. There is a homomorphism $f:\left.\left.E_{1}\right|_{L} \rightarrow \tilde{E}_{2}\right|_{L}$ which is isomorphic at the generic point of $L$. Let $E$ be the coherent sheaf of pure dimension 2 on $Q_{0}$ associated to the triple $\left(E_{1}, \tilde{E}_{2}, f\right)$.

Claim 1. $E$ is stable.

Let $F$ be a coherent subsheaf of $E$ with $0<a_{0}(F)<a_{0}(E)$. We have the following exact commutative diagram with $F^{(1)} \rightarrow E_{1}$ and $\tilde{F}^{(2)} \rightarrow \tilde{E}_{2}$ 
injective:

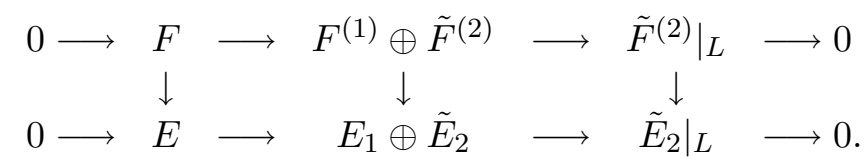

Assume that $\operatorname{rank} F^{(1)}=0$. Then $F \cong \tilde{F}^{(2)}(-L)$. If $\operatorname{rank} \tilde{F}^{(2)}=1$, then we have $c_{1}\left(\tilde{F}^{(2)}(-L)\right) \leq-1<-1 / 2=\mu\left(\tilde{E}_{2}(-L)\right)$ by the stability of $\tilde{E}_{2}(-L)$. Hence we have $\mu^{S}(F) \leq-1<\mu^{S}(E)$. If $\operatorname{rank} \tilde{F}^{(2)}=2, F$ is contained in $\tilde{E}_{2}(-L)$ and

$$
\begin{aligned}
\frac{\chi(F(m))}{2} & \leq \frac{\chi\left(\tilde{E}_{2}(-L)(m)\right)}{2} \\
& =\left(\begin{array}{c}
m+2 \\
2
\end{array}\right)-\frac{m+1}{2}-\frac{c_{2}\left(\tilde{E}_{2}\right)}{2} \\
& <\left(\begin{array}{c}
m+2 \\
2
\end{array}\right)-\frac{m+1}{2}-\frac{c_{2}\left(E_{1}\right)+c_{2}\left(\tilde{E}_{2}\right)}{4}=\frac{\chi(E(m))}{4}
\end{aligned}
$$

for all sufficiently large integers $m$.

Assume that $\operatorname{rank} F^{(1)}=1$. If $\operatorname{rank} \tilde{F}^{(2)}=0$, then we have $\mu^{S}(F)=$ $\mu^{S}\left(F^{(1)}\right) \leq-1<-1 / 2=\mu^{S}\left(E_{1}\right)$ by the stability of $E_{1}$. Hence we have $\mu^{S}(F)<-1 / 2=\mu^{S}(E)$. If $\operatorname{rank} \tilde{F}^{(2)}=1$, we have $\mu^{S}\left(F^{(1)}\right) \leq-1<$ $-1 / 2=\mu^{S}\left(E_{1}\right)$ and $\mu^{S}\left(\tilde{F}^{(2)}\right) \leq 0<1 / 2=\mu^{S}\left(\tilde{E}_{2}\right)$ by the stability of $E_{1}$ and $\tilde{E}_{2}$. Hence we have

$$
\mu^{S}(F)=\frac{\mu^{S}\left(F^{(1)}\right)+\mu^{S}\left(\tilde{F}^{(2)}\right)-1}{2} \leq-1<-\frac{1}{2}=\mu^{S}(E) .
$$

If $\operatorname{rank} \tilde{F}^{(2)}=2$, then

$$
\mu^{S}(F) \leq \frac{\mu^{S}\left(F^{(1)}\right)+2 \mu^{S}\left(\tilde{E}_{2}\right)-2}{3} \leq-2 / 3<-1 / 2=\mu^{S}(E) .
$$

Assume that $\operatorname{rank} F^{(1)}=2$. If $\operatorname{rank} \tilde{F}^{(2)}=0, F$ is contained in the kernel $F^{\prime}$ of the homomorphism $\left.E_{1} \rightarrow \tilde{E}_{2}\right|_{L}$. Since $\left.E_{1} \rightarrow \tilde{E}_{2}\right|_{L}$ is not zero at the generic point of $L$, we have $\mu^{S}(F) \leq\left(2 \mu^{S}\left(E_{1}\right)-1\right) / 2=-1<\mu^{S}(E)$. If $\operatorname{rank} \tilde{F}^{(2)}=1$, we have $\mu^{S}\left(\tilde{F}^{(2)}\right) \leq 0<1 / 2=\mu^{S}\left(\tilde{E}_{2}\right)$ by the stability of $\tilde{E}_{2}$. Hence we have

$$
\mu^{S}(F)=\frac{2 \mu^{S}\left(F^{(1)}\right)+\mu^{S}\left(\tilde{F}^{(2)}\right)-1}{3} \leq-\frac{2}{3}<\mu^{S}(E) .
$$


This completes the proof of the stability of $E$. Hence $E \in M_{(1), Q_{0}}^{P_{n_{1}}^{(-1)}, P_{n_{2}}^{(1)}}(k) \neq$ $\emptyset$. Similarly for integers $n_{1}, n_{2}$ with $n_{1}+n_{2}=n_{0}$ and $0<n_{2}<n_{1}$, $M_{(2), Q_{0}}^{P_{n_{1}}^{(1)}, P_{n_{2}}^{(-1)}} \neq \emptyset$.

Let $n_{1}, n_{2}$ be integers with $n_{1}+n_{2}=n_{0}$ and $n_{1}, n_{2} \geq 0$. We will show that $M_{(1), Q_{0}}^{P_{n_{1}}^{(0)}, P_{n_{2}}^{(0)}}(k) \neq \emptyset$. There exist rank $2 \mu$-semi-stable sheaves $E_{1}$ on $H_{1}, \tilde{E}_{2}$ on $H_{2}$ and a generically injective homomorphism $f:\left.\left.E_{1}\right|_{L} \stackrel{\sim}{\rightarrow} \tilde{E}_{2}\right|_{L}$ such that $\chi\left(E_{1}(m)\right)=P_{n_{1}}^{(0)}(m), \chi\left(\tilde{E}_{2}(m)\right)=P_{n_{2}}^{(0)}(m)$ and either $E_{1}$ or $\tilde{E}_{2}$ is $\mu$-stable. Let $E$ be the coherent sheaf on $Q_{0}$ corresponding to the triple $\left(E_{1}, \tilde{E}_{2}, f\right)$.

Claim 2. $E$ is stable.

Let $F$ be a coherent subsheaf of $E$ with $0<a_{0}(F)<a_{0}(E)$. The following exact commutative diagram is obtained;

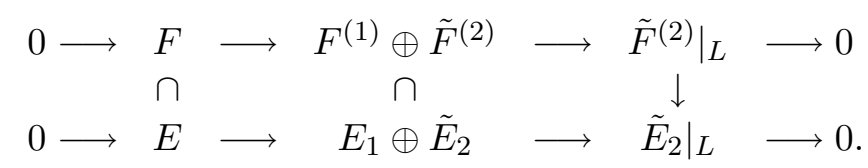

Assume that rank $F^{(1)}=0$. Then $F$ is contained in $\tilde{E}_{2}(-L)$ and so $\mu^{S}(F) \leq$ $\mu^{S}\left(\tilde{E}_{2}(-L)\right)=-1<-1 / 2=\mu^{S}(E)$.

Assume that $\operatorname{rank} F^{(1)}=1$. If $\operatorname{rank} \tilde{F}^{(2)}=0$, then $F=F^{(1)}$ and $F^{(1)} \subset$ $\operatorname{ker}\left(\left.E_{1} \rightarrow \tilde{E}_{2}\right|_{L}\right)$. Thus $\mu^{S}(F)=\mu^{S}\left(F^{(1)}\right) \leq \mu^{S}\left(E_{1}(-L)\right)=-1<\mu^{S}(E)$. If $\operatorname{rank} \tilde{F}^{(2)}=1$, then $\mu^{S}\left(F^{(1)}\right) \leq 0$ and $\mu^{S}\left(\tilde{\tilde{F}}^{(2)}\right) \leq 0$. Moreover $\mu^{S}\left(F^{(1)}\right)<0$ or $\mu^{S}\left(\tilde{F}^{(2)}\right)<0$. Hence we have

$$
\mu^{S}(F)=\frac{\mu^{S}\left(F^{(1)}\right)+\mu^{S}\left(\tilde{F}^{(2)}\right)-1}{2} \leq-1<\mu^{S}(E) .
$$

If $\operatorname{rank} \tilde{F}^{(2)}=2$, then

$$
\mu^{S}(F)=\frac{\mu^{S}\left(F^{(1)}\right)+2 \mu^{S}\left(\tilde{F}^{(2)}\right)-2}{3} \leq-2 / 3<\mu^{S}(E) .
$$

Assume that $\operatorname{rank} F^{(1)}=2$. If $\operatorname{rank} \tilde{F}^{(2)}=0$, then $F=F^{(1)} \subset$ $\operatorname{ker}\left(\left.E_{1} \rightarrow \tilde{E}_{2}\right|_{L}\right)$, and so

$$
\mu^{S}(F)=\mu^{S}\left(F^{(1)}\right) \leq \mu^{S}\left(E_{1}(-L)\right)=-1<\mu^{S}(E) .
$$

If rank $\tilde{F}^{(2)}=1$, then $F^{(1)}$ is contained in the kernel of $\left.E_{1} \rightarrow\left(\tilde{E}_{2} / \tilde{F}^{(2)}\right)\right|_{L}$. Thus $\mu^{S}\left(F^{(1)}\right) \leq-1 / 2$ and

$$
\mu^{S}(F)=\frac{2 \mu^{S}\left(F^{(1)}\right)+\mu^{S}\left(\tilde{F}^{(2)}\right)-1}{3} \leq-\frac{2}{3}<\mu^{S}(E) .
$$


Hence $E$ is a stable sheaf on $Q_{0}$. This implies that $M_{(1), Q_{0}}^{P_{n_{1}}^{(0)}, P_{n_{2}}^{(0)}}(k) \neq \emptyset$. This completes the proof of the theorem.

Remark 5.2. We consider structures of the moduli spaces $M_{(1), Q_{0}}^{P_{n_{1}}^{(0)}, P_{n_{2}}^{(0)}}$, $M_{\left(1, Q_{0}\right.}^{P_{n_{1}}^{(-1)}, P_{n_{2}}^{(1)}}$ and $M_{(2), Q_{0}}^{P_{n_{1}}^{(1)}, P_{n_{2}}^{(-1)}}$ appeared in Theorem 5.1. Let $M_{H_{i}}^{P}$ be the moduli scheme of stable sheaves on $H_{i}$ with Hilbert polynomial $P$ for $i=$ 1,2 .

Assume that $n_{1} \geq 2$ and $n_{2} \geq 2$. From Theorem 2.1, there exist an open subscheme $U_{0}$ of $M_{(1), Q_{0}}^{P_{n_{1}}^{(0)}, P_{n_{2}}^{(0)}}$ and a dominant morphism $\pi_{0}: U_{0} \rightarrow M_{H_{1}}^{P_{n_{1}}^{(0)}} \times$ $M_{H_{2}}^{P_{n_{2}}^{(0)}}$ such that for a point $x$ which corresponds to a triple $\left(E_{1}, \tilde{E}_{2}, f\right)$, $\pi_{0}(x)$ is the point which corresponds to $\left(E_{1}, \tilde{E}_{2}\right)$. Let $\eta$ be a general point of a general fiber of $\pi_{0}$. Then we have

$$
\begin{aligned}
\operatorname{dim}_{\eta} M_{(1), Q_{0}}^{P_{n_{1}}^{(0)}, P_{n_{2}}^{(0)}} & =\operatorname{dim}\left(M_{H_{1}}^{P_{n_{1}}^{(0)}} \times M_{H_{2}}^{P_{n_{2}}^{(0)}}\right)+3 \\
& =4 n_{1}-3+4 n_{2}-3+3=4 n_{0}-3
\end{aligned}
$$

Similarly for $1 \leq n_{1}<n_{2}$ (resp. $1 \leq n_{2}<n_{1}$ ), there exist an open subscheme $U_{1} \subset M_{(1), Q_{0}}^{P_{n_{1}}^{(-1)}, P_{n_{2}}^{(1)}}$ (resp. $\left.U_{2} \subset M_{(2), Q_{0}}^{P_{n_{1}}^{(1)}, P_{n_{2}}^{(-1)}}\right)$ and a dominant morphism $\pi_{1}: U_{1} \rightarrow M_{H_{1}}^{P_{n}^{(-1)}} \times M_{H_{2}}^{P_{n_{2}}^{(1)}}$ (resp. $\pi_{2}: U_{2} \rightarrow M_{H_{1}}^{P_{n_{1}}^{(1)}} \times M_{H_{2}}^{P_{n_{2}}^{(-1)}}$ ). If $\eta_{1}$ is a general point of a general fiber of $\pi_{1}$, then

$$
\begin{aligned}
\operatorname{dim}_{\eta_{1}} M_{(1), Q_{0}}^{P_{n_{1}}^{(-1)}, P_{n_{2}}^{(1)}} & =\operatorname{dim}\left(M_{H_{1}}^{P_{n_{1}}^{(-1)}} \times M_{H_{2}}^{P_{n_{2}}^{(1)}}\right)+7 \\
& =4 n_{1}-4+4 n_{2}-4+7=4 n_{0}-1
\end{aligned}
$$

Similarly for a general point $\eta_{2}$ of the general fiber of $\pi_{2}$, we have

$$
\operatorname{dim}_{\eta_{2}} M_{(2), Q_{0}}^{P_{n_{1}}^{(1)}, P_{n_{2}}^{(-1)}}=4 n_{0}-1
$$

Take $p \in M_{H_{1}}^{P_{n_{1}}^{(0)}} \times M_{H_{2}}^{P_{n_{2}}^{(0)}}$ such that the corresponding sheaves $E_{1}, \tilde{E}_{2}$ are locally free. Then the dimension of the fiber $\pi_{0}^{-1}(p)$ jumps if $L$ is a jumping line of $E_{1}$ and $\tilde{E}_{2}$. See the definition of jumping line for [[13], 2.2]. In particular the coherent sheaf $\mathcal{H}$ mentioned in Remark 2.2 is not locally free in this case. 
Next we will consider relationships with the relative moduli space of stable sheaves on quadric surfaces. Put $\Sigma:=\mathbf{P}\left(H^{0}\left(\mathbf{P}^{3}, \mathcal{O}_{\mathbf{P}^{3}}(2)\right)^{\vee}\right)$. Let $\tilde{Q} \subset \mathbf{P}^{3} \times \Sigma$ be the universal family of quadric surfaces. We will consider the relative moduli space $M_{\tilde{Q} / \Sigma}^{P_{n_{0}}^{(0,0)}}$ of stable sheaves on $\tilde{Q} / \Sigma$ with respect to the polarization $\mathcal{O}_{\tilde{Q}}(1)=\left.\mathcal{O}_{\mathbf{P}_{\Sigma}^{3}}(1)\right|_{\tilde{Q}}$. Take a point $\xi \in \Sigma$ such that $\tilde{Q}_{\xi}=Q_{0}$ is a reducible quadric surface. The following proposition means that $M_{\tilde{Q} / \Sigma}^{P_{n_{0}}^{(0,0)}}$ is smooth over $\Sigma$ at "general points" of $M_{(1), Q_{0}}^{P_{n_{1}}^{(0)}, P_{n_{2}}^{(0)}}$.

Proposition 5.3. If $n_{1} \geq 2$ and $n_{2} \geq 2$, then there exists a locally free stable sheaf $E \in M_{(1), Q_{0}}^{P_{n_{1}}^{(0)}, P_{n_{2}}^{(0)}}$ such that $H^{2}(X, \mathcal{E} n d(E))=0$.

Proof. Take stable bundles $E_{1}$ on $H_{1}$ with $c_{1}\left(E_{1}\right)=0, c_{2}\left(E_{1}\right)=n_{1}$ and $\tilde{E}_{2}$ on $H_{2}$ with $c_{1}\left(\tilde{E}_{1}\right)=0, c_{2}\left(\tilde{E}_{2}\right)=n_{2}$ such that $\left.E_{1}\right|_{L} \cong \mathcal{O}_{L}^{\oplus 2}$ and $\left.\tilde{E}_{2}\right|_{L} \cong \mathcal{O}_{L}^{\oplus 2}$. Take an isomorphism $f:\left.\left.E_{1}\right|_{L} \stackrel{\sim}{\rightarrow} \tilde{E}_{2}\right|_{L}$. Let $E$ be the coherent sheaf on $Q_{0}$ corresponding to the triple $\left(E_{1}, \tilde{E}_{2}, f\right)$. Then $E$ is locally free and stable by the proof of Theorem 5.1. The exact sequence

$$
\left.0 \longrightarrow \mathcal{E} n d(E) \longrightarrow \mathcal{E} n d\left(E_{1}\right) \oplus \mathcal{E} n d\left(\tilde{E}_{2}\right) \longrightarrow \mathcal{E} n d\left(\tilde{E}_{2}\right)\right|_{L} \longrightarrow 0
$$

induces the following exact sequence;

$$
0=H^{1}\left(\mathcal{O}_{L}^{\oplus 4}\right) \longrightarrow H^{2}(\mathcal{E} n d(E)) \longrightarrow H^{2}\left(\mathcal{E} n d\left(E_{1}\right) \oplus \mathcal{E} n d\left(\tilde{E}_{2}\right)\right) \text {. }
$$

Since $E_{1}, \tilde{E}_{2}$ are stable bundles on $\mathbf{P}^{2}, H^{2}\left(\mathcal{E} n d\left(E_{1}\right)\right)=0$ and $H^{2}\left(\mathcal{E} n d\left(\tilde{E}_{2}\right)\right)$ $=0$. Hence $H^{2}(\mathcal{E} n d(E))=0$.

TheOREM 5.4. Assume that $1 \leq n_{1}<n_{2}$. Take a general point $p$ of a general fiber of $\pi_{1}$ in Remark 5.2 and let $E \in M_{(1), Q_{0}}^{P_{n_{1}}^{(-1)}, P_{n_{2}}^{(1)}}$ be the corresponding sheaf. Put $R:=k[[t]]$. Let $t_{0}$ be the closed point of Spec $R$ and $t_{1}$ the generic point of $\operatorname{Spec} R$. Then there exists a morphism $\operatorname{Spec} R \rightarrow \Sigma$ such that $\tilde{Q} \otimes k\left(t_{1}\right)$ is a smooth quadric surface, $\tilde{Q} \otimes k\left(t_{0}\right)=Q_{0}$ and $E$ can be lifted to a coherent sheaf on $\tilde{Q}_{R}$ flat over $R$.

Proof. Take $E \in M_{1}^{P_{n_{1}}^{(-1)}, P_{n_{2}}^{(1)}}$ which is a general point of a general fiber of $\pi_{1}$ and let $\left(E_{1}, \tilde{E}_{2}, f\right)$ be the corresponding triple. We may assume that $E_{1}$ and $\tilde{E}_{2}$ are locally free, $\left.E_{1}\right|_{L} \cong \mathcal{O}_{L} \oplus \mathcal{O}_{L}(-1)$ and $\left.\tilde{E}_{2}\right|_{L} \cong \mathcal{O}_{L} \oplus \mathcal{O}_{L}(1)$. For the homomorphism $f: \mathcal{O}_{L} \oplus \mathcal{O}_{L}(-1) \rightarrow \mathcal{O}_{L} \oplus \mathcal{O}_{L}(1)$, det $f$ can be 
considered as a section of $\mathcal{O}_{L}(2)$. So we may assume that $f(p)$ is isomorphic except at distinct two points $p_{1}, p_{2}$ of $L$. Then coker $f_{p_{i}} \cong k\left(p_{i}\right)$ for $i=1,2$.

On the other hand, there is an exact sequence

$$
\left.\left.\left.\mathcal{E} n d(E) \longrightarrow \mathcal{E} n d(E)\right|_{H_{1}} \oplus \mathcal{E} n d(E)\right|_{H_{2}} \longrightarrow \mathcal{E} n d(E)\right|_{L} \longrightarrow 0 .
$$

Canonical homomorphisms $\left.\mathcal{E} n d(E)\right|_{H_{1}} \rightarrow \mathcal{E} n d\left(E_{1}\right),\left.\mathcal{E} n d(E)\right|_{H_{2}} \rightarrow \mathcal{E} n d\left(\tilde{E}_{2}\right)$ are induced and they are isomorphic on $H_{1} \backslash\left\{p_{1}, p_{2}\right\}, H_{2} \backslash\left\{p_{1}, p_{2}\right\}$ respectively. Since $E_{1}$ and $\tilde{E}_{2}$ are stable bundles on $\mathbf{P}^{2}, H^{2}\left(\mathcal{E} n d\left(E_{1}\right)\right)=$ $H^{2}\left(\mathcal{E} n d\left(\tilde{E}_{2}\right)\right)=0$. Therefore we have $H^{2}\left(\left.\mathcal{E} n d(E)\right|_{H_{1}}\right)=H^{2}\left(\left.\mathcal{E} n d(E)\right|_{H_{2}}\right)=$ 0 . The composition $g:\left.\left.\left.\mathcal{E} n d(E)\right|_{H_{1}} \rightarrow \mathcal{E} n d(E)\right|_{L} \rightarrow \mathcal{E} n d\left(\tilde{E}_{2}\right)\right|_{L}$ induces the following homomorphism:

$$
\psi:\left.\left.\mathcal{E} n d(E)\right|_{H_{1}} \oplus \mathcal{E} n d\left(\tilde{E}_{2}\right) \longrightarrow \mathcal{E} n d\left(\tilde{E}_{2}\right)\right|_{L} ; \quad \psi(a, b):=g(a)-\left.b\right|_{L} .
$$

Then we obtain the following exact commutative diagram:

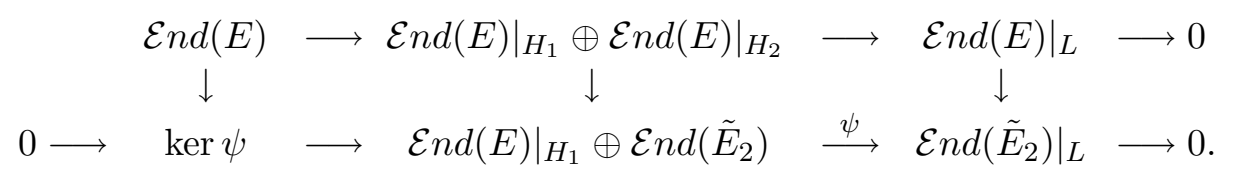

Since $\left.\mathcal{E} n d\left(\tilde{E}_{2}\right)\right|_{L} \cong \mathcal{O}_{L}(-1) \oplus \mathcal{O}_{L}^{\oplus 2} \oplus \mathcal{O}_{L}(1)$, we have $H^{1}\left(\left.\mathcal{E} n d\left(\tilde{E}_{2}\right)\right|_{L}\right)=0$. Thus by the exact sequence

$$
H^{1}\left(\left.\mathcal{E} n d\left(\tilde{E}_{2}\right)\right|_{L}\right) \longrightarrow H^{2}(\operatorname{ker} \psi) \longrightarrow H^{2}\left(\left.\mathcal{E} n d(E)\right|_{H_{1}} \oplus \mathcal{E} n d\left(\tilde{E}_{2}\right)\right),
$$

we have $H^{2}(\operatorname{ker} \psi)=0$. From the construction, $\mathcal{E} n d(E) \rightarrow \operatorname{ker} \psi$ is isomorphic on $Q_{0} \backslash\left\{p_{1}, p_{2}\right\}$. Hence we have $H^{2}(\mathcal{E} n d(E))=0$.

After a suitable projective linear transformation, we may assume that $Q_{0}$ is given by the equation $\{x y=0\}$ in $\mathbf{P}^{3}=\operatorname{Proj} k[x, y, z, w]$. Take $a_{1}:=\alpha z+\beta w \neq 0, a_{2}:=\gamma z+\delta w \neq 0$ with $\alpha, \beta, \gamma, \delta \in k$ such that $a_{1}\left(p_{1}\right)=0$ and $a_{2}\left(p_{2}\right)=0$. Consider $\mathcal{Q}:=\operatorname{Proj} R[x, y, z, w] /\left(x y-t a_{1} a_{2}\right)$. Then $\mathcal{Q}_{t_{0}}=Q_{0}$ and $\mathcal{Q}_{t_{1}}$ is a smooth quadric surface. From Lemma 4.1 and Proposition 4.6, $E$ can be lifted to a coherent sheaf $\tilde{E}$ on $\mathcal{Q}$ flat over $R$.

Conclusion 5.5. Let $\eta \in \Sigma$ be the scheme theoretic generic point. Then we have

$$
M_{\tilde{Q}_{\eta}}^{P_{n}^{(0,0)}}=\coprod_{a \geq 0} M_{a},
$$

where $M_{a} \otimes_{k(\eta)} \overline{k(\eta)}=M\left(2,(a,-a), n_{0}-a^{2}\right) \bigsqcup M\left(2,(-a, a), n_{0}-a^{2}\right)$ for $a>$ $0, M_{0} \otimes_{k(\eta)} \overline{k(\eta)}=M\left(2,(0,0), n_{0}\right)$ and $M(2,(a, b), c)$ is the moduli scheme 
of rank 2 stable sheaves $E$ on $\tilde{Q} \otimes_{k(\eta)} \overline{k(\eta)} \cong \mathbf{P}^{1} \times \mathbf{P}^{1}$ with $c_{1}(E)=(a, b)$ and $c_{2}(E)=c$. Note that $M\left(2,(a,-a), n_{0}-a^{2}\right)$ and $M\left(2,(-a, a), n_{0}-a^{2}\right)$ are contained in the same irreducible component of $M_{\tilde{Q} / \Sigma}^{P_{n_{0}}^{(0,0)}}$, which is the closure of $M_{a}$ in $M_{\tilde{Q} / \Sigma}^{P_{n_{0}}^{(0,0)}}$. If $M\left(2,(a,-a), n_{0}-a^{2}\right) \neq \emptyset$, then $\operatorname{dim} M\left(2,(a,-a), n_{0}-\right.$ $\left.a^{2}\right)=4 n_{0}-3-2 a^{2}$. Thus we can see from the dimension calculation in Remark 5.2 that $M_{\tilde{Q} / \Sigma}^{P_{n_{0}}^{(0,0)}}$ is not flat over $\Sigma$ at "general points" of $M_{1}^{P_{n_{1}}^{(-1)}, P_{n_{2}}^{(1)}}$. On the other hand, Theorem 5.4 means that "general points" in $M_{1}^{P_{n_{1}}^{(-1)}, P_{n_{2}}^{(1)}}$ are contained in the closure of $M_{\tilde{Q}_{\eta}}^{P_{n}^{(0,0)}}$. Such a non-flat relative moduli space appears because $\operatorname{dim} \Sigma \geq 2$. (Compare it with the argument in [[3], section $2]$.) We can see more explicit properties of degeneration of sheaves. Let us consider a degeneration $\mathcal{Q}=\operatorname{Proj} R[x, y, z, w] /\left(x y-t a_{1} a_{2}\right)$ as in Theorem 5.4 such that $a_{1}, a_{2}$ are linearly independent. Let $p_{i}$ be the zero point of $a_{i}$ for $i=1,2$. Take $E \in M_{1}^{P_{n_{1}}^{(-1)}, P_{n_{2}}^{(1)}}$ such that $E_{1}$ and $\tilde{E}_{2}$ are stable bundles, $\left.E_{1}\right|_{L} \cong \mathcal{O}_{L} \oplus \mathcal{O}_{L}(-1),\left.\tilde{E}_{2}\right|_{L} \cong \mathcal{O}_{L} \oplus \mathcal{O}_{L}(1)$ and $f$ is injective, where $\left(E_{1}, \tilde{E}_{2}, f\right)$ is the corresponding triple. The arguments of the proof of Theorem 5.4 conclude that $E$ can be lifted to $\mathcal{Q}$ if and only if $\operatorname{det} f\left(p_{i}\right)=0$ for $i=1,2$. The dimension of

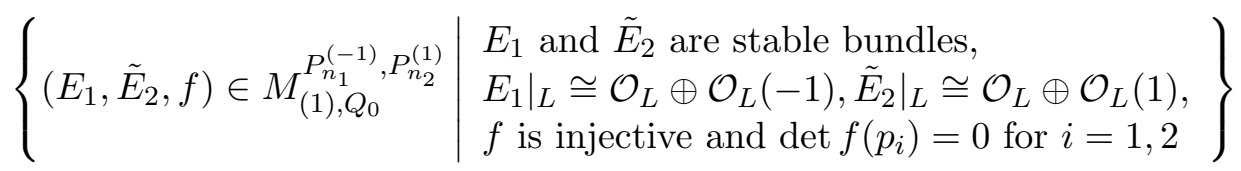

is $4 n_{0}-3$. So it is contained in the closure of $M_{0} \subset M_{\tilde{Q}_{\eta}}^{P_{n_{0}}^{(0,0)}}$.

Next we will consider stable sheaves on $Q_{0}$ with another Hilbert polynomial. We fix a positive integer $n_{0}$ and put $P_{n_{0}}^{(-1,-1)}(m):=4\left(\begin{array}{c}m+2 \\ 2\end{array}\right)-4(m+$ $1)+1-n_{0}$. For an integer $n$, put $P_{n}^{(-2)}(m):=2\left(\begin{array}{c}m+2 \\ 2\end{array}\right)-2(m+1)+1-n$. Put

$$
M_{Q_{0}}^{\circ} P_{n_{0}}^{(-1,-1)}:=\left\{E \in M_{Q_{0}}^{P_{n_{0}}^{(-1,-1)}}|\operatorname{rank} E|_{H_{1}}=\left.\operatorname{rank} E\right|_{H_{2}}=2\right\} .
$$

THEOREM 5.6. Let $n_{0}$ be an integer with $n_{0} \geq 6$. Then $M_{Q_{0}}^{\circ} P_{n_{0}^{(-1,-1)}}^{=}=$

$$
\bigcup_{\substack{n_{1}+n_{2}=n_{0} \\ n_{1}>0, n_{2}>0}} M_{(1), Q_{0}}^{P_{n_{1}}^{(-1)}, P_{n_{2}}^{(-1)}} \cup \bigcup_{\substack{n_{1}+n_{2}=n_{0} \\ 0<n_{1}<n_{2}+1}} M_{(1), Q_{0}}^{P_{n_{1}}^{(-2)}, P_{n_{2}}^{(0)}} \cup \bigcup_{\substack{n_{1}+n_{2}=n_{0} \\ n_{1}+1>n_{2}>0}} M_{(2), Q_{0}}^{P_{n_{1}}^{(0)}, P_{n_{2}}^{(-2)}} .
$$


Moreover each component of the right hand side is non-empty.

Proof. Let $E$ be an element of $M^{\circ}{ }_{Q_{0}}^{(-1,-1)}(k)$. Then

$$
\begin{aligned}
\chi(E(m))=4 & \left(\begin{array}{c}
m+2 \\
2
\end{array}\right)+\left(c_{1}\left(E^{(1)}\right)+c_{1}\left(\tilde{E}^{(2)}\right)-2\right)(m+1)-c_{2}\left(E^{(1)}\right) \\
& -c_{2}\left(\tilde{E}^{(2)}\right)+\frac{c_{1}\left(E^{(1)}\right)^{2}+c_{1}\left(E^{(1)}\right)+c_{1}\left(\tilde{E}^{(2)}\right)^{2}-c_{1}\left(\tilde{E}^{(2)}\right)}{2} .
\end{aligned}
$$

If we put $a:=c_{1}\left(E^{(1)}\right)$, then $c_{1}\left(\tilde{E}^{(2)}\right)=-a-2$. Assume that $a<-2$. Then $c_{1}\left(\tilde{E}^{(2)}(-L)\right)=-a-4>-2$ and so $\mu^{S}\left(\tilde{E}^{(2)}(-L)\right)>-1=\mu^{S}(E)$. Since $\tilde{E}^{(2)}(-L)$ is a subsheaf of $E$, this contradicts the stability of $E$. Assume that $a>0$. Then $c_{1}\left(E^{(1)}(-L)\right)=a-2>-2$. So $E^{(1)}(-L)$ is a subsheaf of $E$ with $\mu^{S}\left(E^{(1)}(-L)\right)>-1=\mu^{S}(E)$ which contradicts the stability of $E$. Hence we have $-2 \leq a \leq 0$.

Case 1. $a=-2$.

If we put $n_{1}=c_{2}\left(\tilde{E}^{(1)}\right)$ and $n_{2}=c_{2}\left(E^{(2)}\right)$, then $n_{1}+n_{2}=n_{0}$ and $E \in M_{(1), Q_{0}}^{P_{n_{1}}^{(-2)}, P_{n_{2}}^{(0)}}$. Since $E^{(1)}$ is a quotient sheaf of $E$, the stability of $E$ implies that

$$
\frac{\chi(E(m))}{4}<\frac{\chi\left(E^{(1)}(m)\right)}{2} \text { for } m \gg 0 .
$$

Hence we have $n_{1}<n_{2}+1$. Since $E^{(1)}$ is a quotient sheaf of $E$ with $\mu^{S}\left(E^{(1)}\right)=-1=\mu^{S}(E), E^{(1)}$ is $\mu$-semi-stable. Thus $n_{1} \geq 1$.

Case 2. $a=0$.

Assume that the homomorphism $\left.\left.E^{(1)}\right|_{L} \rightarrow \tilde{E}^{(2)}\right|_{L}$ is not isomorphic at the generic point of $L$. Then the kernel $F$ of the homomorphism $E^{(1)} \rightarrow$ $\left.\tilde{E}^{(2)}\right|_{L}$ is a subsheaf of $E$ and $c_{1}(F) \geq-1$. So $\mu^{S}(F) \geq-1 / 2>\mu^{S}(E)$ which contradicts the stability of $E$. Thus $\left.\left.E^{(1)}\right|_{L} \rightarrow \tilde{E}^{(2)}\right|_{L}$ is generically isomorphic. Consider the triple $\left(\tilde{E}^{(1)}, E^{(2)}, f\right)\left(f:\left.\left.E^{(2)}\right|_{L} \rightarrow \widetilde{E}^{(1)}\right|_{L}\right)$ corresponding to $E$. Then $c_{1}\left(\tilde{E}^{(1)}\right)=0$ and $c_{1}\left(E^{(2)}\right)=-2$. Hence $E \in M_{2}^{P_{n_{1}}^{(0)}, P_{n_{2}}^{(-2)}}$ where $n_{1}=c_{2}\left(\tilde{E}^{(1)}\right)$ and $n_{2}=c_{2}\left(E^{(2)}\right)$. The proof of Case 1 implies that $n_{1}+1>n_{2}>0$ and $n_{1}+n_{2}=n_{0}$.

Case 3. $a=-1$.

If we put $n_{1}=c_{2}\left(E^{(1)}\right)$ and $n_{2}=c_{2}\left(\tilde{E}^{(2)}\right)$, then $n_{1}+n_{2}=n_{0}$ and

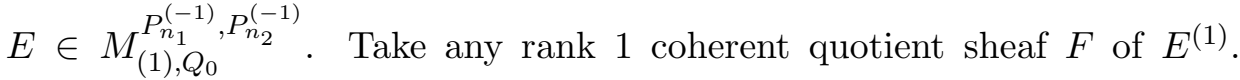
The stability of $E$ implies that $c_{1}(F)=\mu^{S}(F)>\mu^{S}(E)=-1$. Thus $\mu^{S}(F)=c_{1}(F) \geq 0>\mu^{S}\left(E^{(1)}\right)$. Hence $E^{(1)}$ is stable and so $n_{1}>0$. Take 
any rank 1 coherent quotient sheaf $F$ of $\tilde{E}^{(2)}$. Let $F^{\prime}$ be the image of $E \rightarrow F$. Then $\mu^{S}(E)=-1<\mu^{S}\left(F^{\prime}\right) \leq \mu^{S}(F)$ and so $\mu^{S}(F) \geq 0>\mu^{S}\left(\tilde{E}^{(2)}\right)$. Hence $\tilde{E}^{(2)}$ is stable and $n_{2}>0$. This completes the proof of the first part of the theorem.

Take integers $n_{1}, n_{2}$ with $n_{1}+n_{2}=n_{0}$ and $0<n_{1}<n_{2}+1$. We will show that $M_{(1), Q_{0}}^{P_{n_{1}}^{(-2)}, P_{n_{2}}^{(0)}} \neq \emptyset$. If $n_{1} \neq 1,2$, then there exist rank $2 \mu$-stable bundles $E_{1}$ on $H_{1}$ with $\chi\left(E_{1}(m)\right)=P_{n_{1}}^{(-2)}(m), \tilde{E}_{2}$ on $H_{2}$ with $\chi\left(\tilde{E}_{2}(m)\right)=$ $P_{n_{2}}^{(0)}(m)$ and an injective homomorphism $f:\left.\left.E_{1}\right|_{L} \rightarrow \tilde{E}_{2}\right|_{L}$. If $n_{1}=1$ or 2 , then there exist a rank $2 \mu$-semi-stable sheaf $E_{1}$ on $H_{1}$ with $\chi\left(E_{1}(m)\right)=$ $P_{n_{1}}^{(-2)}(m)$, a rank $2 \mu$-stable sheaf $\tilde{E}_{2}$ on $H_{2}$ with $\chi\left(\tilde{E}_{2}(m)\right)=P_{n_{2}}^{(0)}(m)$ and a generically injective homomorphism $f:\left.\left.E_{1}\right|_{L} \rightarrow \tilde{E}_{2}\right|_{L}$. Let $E$ be the coherent sheaf of pure dimension 2 on $Q_{0}$ corresponding to the triple $\left(E_{1}, \tilde{E}_{2}, f\right)$.

Claim 1. $E$ is stable

Take any coherent subsheaf $F$ of $E$ with $0<a_{0}(F)<a_{0}(E)$. The following exact commutative diagram is obtained:

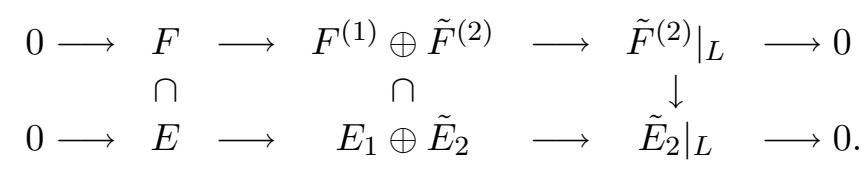

Assume that $F^{(1)}=0$. Then $F=\tilde{F}^{(2)}(-L)$. If $\operatorname{rank} \tilde{F}^{(2)}=1$, then $\mu^{S}(F)<$ $\mu^{S}\left(\tilde{E}_{2}(-L)\right)=-1=\mu^{S}(E)$. If $\operatorname{rank} \tilde{F}^{(2)}=2$, then for all sufficiently large integers $m$,

$$
\begin{aligned}
\frac{\chi(F(m))}{2} & \leq \frac{\chi\left(\tilde{E}_{2}(-L)(m)\right)}{2} \\
& =\left(\begin{array}{c}
m+2 \\
2
\end{array}\right)-(m+1)-\frac{n_{2}}{2} \\
& <\left(\begin{array}{c}
m+2 \\
2
\end{array}\right)-(m+1)+\frac{1-n_{0}}{4}=\frac{\chi(E(m))}{4} .
\end{aligned}
$$

Assume that $\operatorname{rank} F^{(1)}=1$. If $\operatorname{rank} \tilde{F}^{(2)}=0$, then $F=F^{(1)} \subset \operatorname{ker}\left(E_{1} \rightarrow\right.$ $\left.\left.\tilde{E}_{2}\right|_{L}\right)$. Since $\left.\left.E_{1}\right|_{L} \rightarrow \tilde{E}_{2}\right|_{L}$ is generically injective, $\mu^{S}\left(E_{1}(-L)\right)=\mu^{S}\left(\operatorname{ker}\left(E_{1}\right.\right.$ $\left.\left.\left.\rightarrow \tilde{E}_{2}\right|_{L}\right)\right)$. Thus $\mu^{S}(F)=\mu^{S}\left(F^{(1)}\right) \leq \mu^{S}\left(E_{1}(-L)\right)=-2<\mu^{S}(E)$. If $\operatorname{rank} \tilde{F}^{(2)}=1$, then $\mu^{S}\left(\tilde{F}^{(2)}\right)<\mu^{S}\left(\tilde{E}_{2}\right)=0$. Thus

$$
\mu^{S}(F)=\frac{\mu^{S}\left(F^{(1)}\right)+\mu^{S}\left(\tilde{F}^{(2)}\right)-1}{2}
$$




$$
\leq \frac{-1-1-1}{2}<-1=\mu^{S}(E) .
$$

Assume that $\operatorname{rank} \tilde{F}^{(2)}=2$. If $n_{1} \neq 1,2$, then $E_{1}$ is $\mu$-stable and so $\mu^{S}\left(F^{(1)}\right)<\mu^{S}\left(E_{1}\right)=-1$. Hence

$$
\mu^{S}(F)=\frac{\mu^{S}\left(F^{(1)}\right)+2 \mu^{S}\left(\tilde{F}^{(2)}\right)-2}{3} \leq \frac{-2-2}{3}<-1=\mu^{S}(E) .
$$

If $n_{1}=1$, then $E_{1} \cong \mathcal{O}_{H_{1}}(-1)^{\oplus 2}$ and so

$$
\chi\left(F^{(1)}(m)\right) \leq \chi\left(\mathcal{O}_{H_{1}}(m-1)\right)=\left(\begin{array}{c}
m+2 \\
2
\end{array}\right)-(m+1)
$$

for all sufficiently large integers $m$. If $n_{1}=2$, then $E_{1}^{\vee \vee} \cong \mathcal{O}_{H_{1}}(-1)^{\oplus 2}$ and $E_{1}^{\vee \vee} / E_{1} \cong k(p)$ for some $p \in H_{1}$. Thus $\chi\left(F^{(1)}(m)\right) \leq \chi\left(\mathcal{O}_{H_{1}}(m-1)\right)$ for $m \gg 0$. Moreover $n_{1}=1$ implies $n_{2} \geq 5$ and $n_{1}=2$ does $n_{2} \geq 4$. Hence for $n_{1}=1,2$,

$$
\begin{aligned}
\frac{\chi(F(m))}{3} & \leq \frac{\chi\left(F^{(1)}(m)\right)+2 \chi\left(\tilde{E}_{2}(m)\right)-2 \chi\left(\left.\tilde{E}_{2}\right|_{L}(m)\right)}{3} \\
& \leq \frac{\left(\begin{array}{c}
m+2 \\
2
\end{array}\right)-(m+1)+2\left(\begin{array}{c}
m+2 \\
2
\end{array}\right)-2(m+1)-n_{2}}{3} \\
& =\left(\begin{array}{c}
m+2 \\
2
\end{array}\right)-(m+1)-\frac{n_{2}}{3} \\
& <\left(\begin{array}{c}
m+2 \\
2
\end{array}\right)-(m+1)+\frac{1-n_{1}-n_{2}}{4}=\frac{\chi(E(m))}{4}
\end{aligned}
$$

for all sufficiently large integers $m$.

Assume that $\operatorname{rank} F^{(1)}=2$. If $\operatorname{rank} \tilde{F}_{2}=0$, then $F=F^{(1)} \subset \operatorname{ker}\left(E_{1} \rightarrow\right.$ $\left.\left.\tilde{E}_{2}\right|_{L}\right)$. Since $E_{1}(-L) \rightarrow \operatorname{ker}\left(\left.E_{1} \rightarrow \tilde{E}_{2}\right|_{L}\right)$ is isomorphic in codimension 1, $\mu^{S}(F) \leq \mu^{S}\left(E_{1}(-L)\right)=-2<\mu^{S}(E)$. If $\operatorname{rank} \tilde{F}_{2}=1$, then $\mu^{S}\left(\tilde{F}_{2}\right)<$ $\mu^{S}\left(\tilde{E}_{2}\right)=0$. Hence

$$
\mu^{S}(F) \leq \frac{2 \mu^{S}\left(E_{1}\right)+\mu^{S}\left(\tilde{F}_{2}\right)-1}{3} \leq-\frac{4}{3}<-1=\mu^{S}(E) .
$$

Hence $E$ is a stable sheaf on $Q_{0}$.

We have proved Claim 1 and so we have $M_{(1), Q_{0}}^{P_{n_{1}}^{(-2)}, P_{n_{2}}^{(0)}} \neq \emptyset$. Similarly for $n_{1}, n_{2} \in \mathbf{Z}$ with $0<n_{2}<n_{1}+1$, we have $M_{(2), Q_{0}}^{P_{n}^{(0)}, P_{n_{2}}^{(-2)}} \neq \emptyset$. 
Let $n_{1}, n_{2}$ be integers with $n_{1}>0, n_{2}>0$ and $n_{1}+n_{2}=n_{0}$. We will prove that $M_{(1), Q_{0}}^{P_{n_{1}}^{(-1)}, P_{n_{2}}^{(-1)}} \neq \emptyset$. There are rank 2 stable bundles $E_{1}$ on $H_{1}$ with $\chi\left(E_{1}(m)\right)=2\left(\begin{array}{c}m+2 \\ 2\end{array}\right)-(m+1)-n_{1}, \tilde{E}_{2}$ on $H_{2}$ with $\chi\left(\tilde{E}_{2}(m)\right)=$ $2\left(\begin{array}{c}m+2 \\ 2\end{array}\right)-(m+1)-n_{2}$, and an injective homomorphism $f:\left.\left.E_{1}\right|_{L} \rightarrow \tilde{E}_{2}\right|_{L}$. Let $E$ be the coherent sheaf on $Q_{0}$ corresponding to the triple $\left(E_{1}, \tilde{E}_{2}, f\right)$. Claim 2. $E$ is stable.

Let $F$ be a coherent subsheaf of $E$ with $0<a_{0}(F)<a_{0}(E)$. Assume that $\operatorname{rank} F^{(1)}=0$. Then $F=\tilde{F}^{(2)}(-L) \subset \tilde{E}_{2}(-L)$. Thus $\mu^{S}(F) \leq$ $\mu^{S}\left(\tilde{E}_{2}(-L)\right)=-3 / 2<-1=\mu^{S}(E)$. Assume that $\operatorname{rank} F^{(1)}=1$. If $\operatorname{rank} \tilde{F}^{(2)}=0$, then $F=F^{(1)} \subset \operatorname{ker}\left(\left.E_{1} \rightarrow \tilde{E}_{2}\right|_{L}\right)=E_{1}(-L)$. Thus $\mu^{S}(F) \leq$ $\mu^{S}\left(E_{1}(-L)\right)=-3 / 2<-1=\mu^{S}(E)$. If $\operatorname{rank} \tilde{F}^{(2)} \geq 1$, then

$$
\begin{aligned}
\mu^{S}(F) & =\frac{\mu^{S}\left(F^{(1)}\right)+\operatorname{rank} \tilde{F}^{(2)}\left(\mu^{S}\left(\tilde{F}^{(2)}\right)-1\right)}{1+\operatorname{rank} \tilde{F}^{(2)}} \\
& \leq \frac{-1-3 \operatorname{rank} \tilde{F}^{(2)} / 2}{1+\operatorname{rank} \tilde{F}^{(2)}}<-1=\mu^{S}(E) .
\end{aligned}
$$

Assume that $\operatorname{rank} F^{(1)}=2$. If $\operatorname{rank} \tilde{F}^{(2)}=0$, then $F=F^{(1)} \subset \operatorname{ker}\left(E_{1} \rightarrow\right.$ $\left.\left.\tilde{E}_{2}\right|_{L}\right)=E_{1}(-L)$ Thus $\mu^{S}(F) \leq \mu^{S}\left(E_{1}(-L)\right)=-3 / 2<-1=\mu^{S}(E)$. If $\operatorname{rank} \tilde{F}^{(2)}=1$, then the commutative diagram

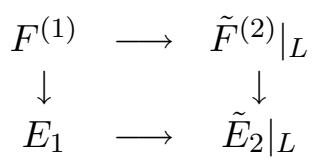

implies that $\mu^{S}\left(F^{(1)}\right) \leq \mu^{S}\left(E_{1}\right)-1 / 2=-1$. Hence

$$
\mu^{S}(F)=\frac{2 \mu^{S}\left(F^{(1)}\right)+\mu^{S}\left(\tilde{F}^{(2)}\right)-1}{3} \leq \frac{-2-1-1}{3}<-1=\mu^{S}(E) .
$$

Thus $E$ is a stable sheaf on $Q_{0}$. Hence $M_{(1), Q_{0}}^{P_{n_{1}}^{(-1)}, P_{n_{2}}^{(-1)}} \neq \emptyset$.

Remark 5.7. Similar calculations to Remark 5.2 show that at a "general" point $p$ of $M_{1}^{P_{n_{1}}^{(-1)}, P_{n_{2}}^{(-1)}}, \operatorname{dim}_{p} M_{1}^{P_{n_{1}}^{(-1)}, P_{n_{2}}^{(-1)}}=4 n_{1}-4+4 n_{2}-4+3=$ $4 n_{0}-5$. Similarly at a "general" point $p$ of $M_{1}^{P_{n_{1}}^{(-2)}, P_{n_{2}}^{(0)}}, \operatorname{dim}_{p} M_{1}^{P_{n_{1}}^{(-2)}, P_{n_{2}}^{(0)}}=$ $4 n_{1}-7+4 n_{2}-3+7=4 n_{0}-3$.

Similar arguments to Proposition 5.3 conclude the following proposition. 
Proposition 5.8. For $n_{1} \geq 1$ and $n_{2} \geq 1$, there exists a locally free stable sheaf $E \in M_{(1), Q_{0}}^{P_{n_{1}}^{(-1)}, P_{n_{2}}^{(-1)}}$ such that $H^{2}(X, \mathcal{E} n d(E))=0$.

Remark 5.9. Consider a degeneration $\mathcal{Q}=\operatorname{Proj}(R[x, y, z, w] /(x y-$ $\left.\left.t a_{1} a_{2}\right)\right)$ as in the proof of Theorem 5.4. We assume that $a_{1}, a_{2}$ are linearly independent. Let $p_{i} \in L$ be the zero point of $a_{i}$. Let $\bar{t}_{1}$ be a generic geometric point of $\operatorname{Spec} R$. There is a decomposition of a moduli space of stable sheaves on a smooth quadric surface;

$$
M_{\mathcal{Q}_{\bar{t}_{1}}}^{P_{n_{0}}^{(-1,-1)}}=\coprod_{a \in \mathbf{Z}} M\left(2,(a-1,-a-1), n_{0}-a^{2}\right) .
$$

If $M\left(2,(a-1,-a-1), n_{0}-a^{2}\right) \neq \emptyset$, then $\operatorname{dim} M\left(2,(a-1,-a-1), n_{0}-\right.$ $\left.a^{2}\right)=4 n_{0}-5-2 a^{2}$. From Proposition 5.8, there exists an open subscheme $U \subset M_{\mathcal{Q} / R}^{P_{n_{0}}^{(-1,-1)}}$ smooth over $R$ such that $U_{\bar{\eta}} \subset M\left(2,(-1,-1), n_{0}\right)$ and $U_{t_{0}} \subset M_{(1), Q_{0}}^{P_{n_{1}}^{(-1)}, P_{n_{2}}^{(-1)}}$. We may assume that $E$ is locally free for any $E \in U_{t_{0}}$ and that $\left.E\right|_{H_{1}},\left.E\right|_{H_{2}}$ are stable. There exist a scheme $V$ over $R$ and a morphism of functors $\theta: V \rightarrow \mathcal{M}_{\mathcal{Q} / R}^{P_{n_{0}}^{(-1,-1)}}$ such that the induced morphism $\theta^{\prime}: V \rightarrow M_{\mathcal{Q} / R}^{P_{n_{0}}^{(-1,-1)}}$ is étale and that the image is $U$. On the other hand, let $\tilde{I}_{x, a_{1}}$ be the image of the homomorphism $\mathcal{O}_{\mathcal{Q}}(-1)^{\oplus 2} \rightarrow \mathcal{O}_{\mathcal{Q}}$ defined by the sections $x, a_{1}$. Then $\mathcal{O}_{\mathcal{Q}} / \tilde{I}_{x, a_{1}}$ is flat over $R$. Let $\tilde{E}$ be a flat family of stable bundles on $\mathcal{Q} \times{ }_{R} V / V$ corresponding to $\theta$. Then $\tilde{E} \otimes \tilde{I}_{x, a_{1}}(1)$ is a flat family of stable sheaves whose fiber over $V_{\bar{\eta}}$ is contained in $M\left(2,(-1,1), n_{0}-1\right)$ and whose fiber over $V_{t_{0}}$ is contained in $M_{(1), Q_{0}}^{P_{n_{1}}^{(-1)}, P_{n_{2}}^{(1)}}$.

Let us consider an open subscheme

$$
W:=\left\{\begin{array}{l|l}
\left(E_{1}, \tilde{E}_{2}, f\right) \in M_{1}^{P_{n_{1}}^{(-1)}, P_{n_{2}}^{(1)}} & \begin{array}{l}
E_{1} \text { and } \tilde{E}_{2} \text { are stable bundles, } \\
\left.E_{1}\right|_{L} \cong \mathcal{O}_{L} \oplus \mathcal{O}_{L}(-1) \\
\left.\tilde{E}_{2}\right|_{L} \cong \mathcal{O}_{L} \oplus \mathcal{O}_{L}(1) \\
f:\left.\left.E_{1}\right|_{L} \rightarrow \tilde{E}_{2}\right|_{L} \text { is injective }
\end{array}
\end{array}\right\}
$$

of $M_{(1), Q_{0}}^{P_{n_{1}}^{(-1)}, P_{n_{2}}^{(1)}}$. Then the set $W^{\prime}:=\left\{\tilde{E} \otimes \tilde{I}_{x, a_{1}}(1) \otimes k(s)\right\}_{s \in V_{t_{0}}}$ is contained in $W$ and

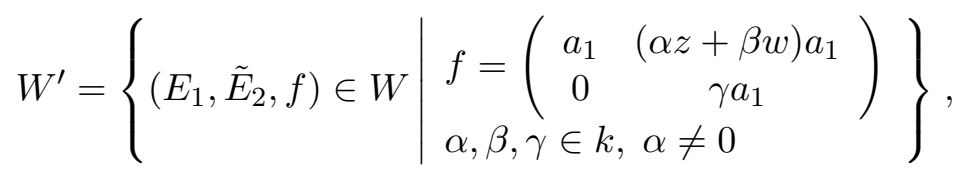


where $f: \mathcal{O}_{L} \oplus \mathcal{O}_{L}(-1)=\left.\left.E_{1}\right|_{L} \rightarrow \tilde{E}_{2}\right|_{L}=\mathcal{O}_{L} \oplus \mathcal{O}_{L}(1)$ is regarded as a matrix $\left(\begin{array}{ll}b_{1} & b_{2} \\ b_{3} & b_{4}\end{array}\right)$ with $b_{1} \in H^{0}\left(\mathcal{O}_{L}(1)\right), b_{2} \in H^{0}\left(\mathcal{O}_{L}(2)\right), b_{3} \in k$ and $b_{4} \in H^{0}\left(\mathcal{O}_{L}(1)\right)$. Moreover $\operatorname{dim} M\left(2,(-1,1), n_{0}-1\right)=\operatorname{dim} W^{\prime}=4 n_{0}-5$.

\section{REFERENCES}

[1] A. Altman and S. Kleiman, Compactifying the Picard scheme, Adv. in Math., 35 (1980), no. 1, 50-112.

[2] G. Faltings, Moduli-stacks for bundles on semistable curves, Math. Ann., 304 (1996), 489-515.

[3] D. Gieseker and J. Li, Irreducibility of moduli of rank-2 vector bundles on Algebraic surfaces, J. Differential Geom., 40 (1994), 23-104.

[4] A. Grothendieck, Éléments de géométrie algébrique, Chaps. I,II,III,IV, Inst. Hautes Études Sci. Publ. Math. No. 4,8,11,17,20,24,28,32, 1960-1967.

[5] M. Inaba, Moduli of parabolic stable sheaves on a projective scheme, J. Math. Kyoto Univ ., 40-1 (2000), 119-136.

[6] _ On the moduli of stable sheaves on some nonreduced projective schemes, (to appear in J. Alg. Geometry).

[7] A. Langer, Semistable sheaves in positive characteristic, (to appear).

[8] M. Maruyama, Moduli of stable sheaves, II, J. Math. Kyoto Univ., 18 (1978), no. 3, $557-614$

[9] _ Construction of moduli spaces of stable sheaves via Simpson's idea, Lecture Notes in Pure and Appl. Math. 179, Dekker, New York, (1996).

[10] D. Mumford, Geometric invariant theory, Springer-Verlag, Berlin Heidelberg New York, 1965.

[11] D. S. Nagaraj and C. S. Seshadri, Degenerations of the moduli spaces of vector bundles on curves, I, Proc. Indian Acad. Sci., 107 (1997), no. 2, 101-137.

[12] T. Oda and C. S. Seshadri, Compactification of the generalized Jacobian variety, Trans. Amer. Math. Soc., 253 (1979), 1-90.

[13] C. Okonek, M. Schneider, H. Spindler, Vector bundles on complex projective spaces, Birkhäuser, Boston, 1980.

[14] A. N. Rudakov, A description of Chern classes of semistable sheaves on a quadric surface, J. Reine Angew. Math., 453 (1994), 113-135.

[15] C. S. Seshadri, Geometric reductivity over arbitrary base, Adv. in Math., 26 (1977), no. $3,225-274$.

[16] — Fibrés vectoriels sur les courbes algébriques, Astérisque, 96, Société Mathématique de France, Paris, 1982.

[17] C. T. Simpson, Moduli of representations of the fundamental group of a smooth projective variety I, Inst. Hautes Études Sci., 79 (1994), 47-129.

[18] S. Soberon-Chavez, Rank 2 vector bundles over a complex quadric surface, Quart. J. Math. Oxford (2), 36 (1985), 159-172. 
[19] K. Yokogawa, Compactification of moduli of parabolic sheaves and moduli of parabolic Higgs sheaves, J. Math. Kyoto Univ., 33 (1993), no. 2, 451-504.

[20] H. Xia, Degenerations of moduli of stable bundles over algebraic curves, Compos. Math., 98 (1995), 305-330.

Department of Mathematics

Faculty of Science

Kyoto University

Kyoto 606-8502, Japan

inaba@kusm.kyoto-u.ac.jp 